\title{
An extension of Birnbaum-Saunders distributions based on scale mixtures of skew-normal distributions with applications to regression models
}

\author{
Rocío Paola Maehara Sánchez
}

\author{
DissertaÇÃo/TeSE APRESEnTAda \\ $\mathrm{AO}$ \\ Instituto De Matemática e Estatística \\ DA \\ Universidade De SÃo Paulo \\ PARA \\ OBTENÇÃO DO TÍTULO \\ $\mathrm{DE}$ \\ Doutor EM CIÊNCIAS \\ Programa: Estatística \\ Orientador: Prof. Dr. Heleno Bolfarine \\ Coorientador: Prof. Dr. Filidor Vilca Labra
}

Durante o desenvolvimento deste trabalho o autor recebeu auxílio financeiro de CAPES e CNPq 
An extension of Birnbaum-Saunders distributions based on scale mixtures of skew-normal distributions with applications to regression models

Esta é a versão original da tese elaborada pela candidata Rocío Paola Maehara Sánchez, tal como submetida à Comissão Julgadora. 


\section{An extension of Birnbaum-Saunders distributions based on scale mixtures of skew-normal distributions with applications to regression models}

\footnotetext{
Esta versão da dissertação/tese contém as correções e alterações sugeridas pela Comissão Julgadora durante a defesa da versão original do trabalho, realizada em 06/04/2018. Uma cópia da versão original está disponível no Instituto de Matemática e Estatística da Universidade de São Paulo.
}

Comissão Julgadora:

- Prof. Dr. Heleno Bolfarine - IME-USP

- Prof. Dr. Filidor Vilca Labra - IMECC-UNICAMP

- Prof. Dr. Caio Lucidius Naberezny Azevedo - IMECC-UNICAMP

- Prof. Dr. Edwin Moiss Marcos Ortega ESALQ - USP

- Prof. Dr. Helton Saulo Bezerra dos Santos - IME-UFG 


\section{Agradecimentos}

- A Jesus, meu Senhor e Salvador, pela sua presena constante na minha vida, pelo auxílio nas minhas escolhas e me confortar nas horas difíceis.

- Ao meu esposo Luis, pelo amor e paciência nos meus "maus" momentos. Amo você demais! Graças a sua presena foi mais fácil transpor os dias de desânimo e cansaço!

- Aos meus pais, Víctor e Doris, por todo amor, carinho e apoio incondicionais, Amo muito vocês!

- Ao meu irmão Yoshitomi, pelo apoio e compreensão.

- Ao meu orientador, Professor Heleno Bolfarine por seu exemplo como excelente pesquisador e pessoa que me faz sentir motivada a seguir o caminho de ensino e pesquisa.

- Ao meu coorientador, Professor Filidor Edilfonso Vilca Labra, pelos importantes ensinamentos, pela amizade e apoio, e pelo conforto nas horas difíceis.

- Ao Professor Víctor Hugo Lachos Dávila e sua famila pelo apoio, carinho e amizade.

- Aos professores do Departamento de Estatística do IME-USP, pelos importantes ensinamentos concedidos.

- A CAPES e a CNPq, pelo apoio financeiro para a execução desse projeto. 


\section{Resumo}

MAEHARA, R. P. Uma extensão da distribuição Birnbaum-Saunders baseado na misturas de escala skew-normal com aplicações a modelos de regressão. 2018. 109 f. Tese (Doutorado) - Instituto de Matemática e Estatística, Universidade de São Paulo, São Paulo, 2010.

O objetivo deste trabalho é apresentar um estudo de inferência e diagnóstico em uma extensão da família de distribuições de tempos de vida proposta por Birnbaum and Saunders (1969a,b). Esta extensão é obtida ao considerar uma distribuição skew-elíptica em lugar da distribuição normal. Especificamente, neste trabalho desenvolveremos um tipo de distribuição Birnbaum-Saunders (BS) baseda nas distribuições mistura de escala skew-normal (MESN).

Esta família resultante de distribuições de tempos de vida representa uma extensão robusta da distribuição BS usual. Baseado nesta família, vamos reproduzir as propriedades usuais da distribuição BS, e apresentar um método de estimação baseado no algoritmo EM. Além disso, vamos apresentar modelos de regressão associado à distribuições BS (baseada na distribuição mistura de escala skew-normal), que é desenvolvida como uma extensão da distribuição senh-normal (Rieck and Nedelman, 1991), para estes vamos consiserar um estudo de estimação e diagnóstisco para dados sem censura.

Palavras-chave: Distribuição Birnbaum-Saunders, Distribuição skew-normal, Algoritmo EM, Distribuição misturas de escala skew-normal, Regressão não linear, Estimação robusta, Distribuição skew-normal/Independente, Distribuição senh-normal, Análise de influência, eliminação de casos, influência local. 


\section{Abstract}

SOBRENOME, A. B. C. An extension of Birnbaum-Saunders distributions based on scale mixtures of skew-normal distributions with applications to regression models. 2010. 109 f. Tese (Doutorado) - Instituto de Matemática e Estatística, Universidade de São Paulo, São Paulo, 2010.

The aim of this work is to present an inference and diagnostic study of an extension of the lifetime distribution family proposed by Birnbaum and Saunders (1969a,b). This extension is obtained by considering a skew-elliptical distribution instead of the normal distribution. Specifically, in this work we develop a Birnbaum-Saunders (BS) distribution type based on scale mixtures of skew-normal distributions (SMSN).

The resulting family of lifetime distributions represents a robust extension of the usual BS distribution. Based on this family, we reproduce the usual properties of the BS distribution, and present an estimation method based on the EM algorithm. In addition, we present regression models associated with the BS distributions (based on scale mixtures of skew-normal), which are developed as an extension of the sinh-normal distribution (Rieck and Nedelman, 1991)). For this model we consider an estimation and diagnostic study for uncensored data.

Keywords: Birnbaum-Saunders distribution, Skew-normal distribution, EM-algorithm, Scale mixtures of skew-normal distributions, Nonlinear regression, Robust estimation, Skew-normal/Independent distribution, Sinh-normal distribution, Influence analysis, case-deletion, local influence. 


\section{Contents}

$\begin{array}{ll}\text { List of Figures } & \text { ix }\end{array}$

List of Tables $\quad$ xi

1 Introduction $\quad 1$

1.0.1 Birnbaum-Saunders model . . . . . . . . . . . . . . . . . 3

1.0.2 The scale mixtures of skew-normal distribution . . . . . . . . . . . 4

1.0 .3 Local influence . . . . . . . . . . . . . . . . . . . . . . 7

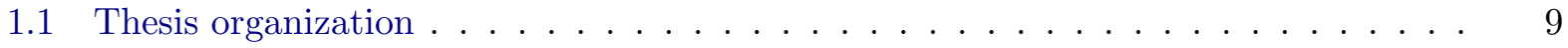

2 Modeling extreme percentiles in Birnbaum-Saunders distributions based on an asymmetric heavy-tailed distribution $\quad 11$

2.1 Introduction . . . . . . . . . . . . . . . . . . . . . 12

2.1.1 Motivating Examples . . . . . . . . . . . . . . . . . . 13

2.2 The SMSN-BS distribution . . . . . . . . . . . . . . . . . . . . . 14

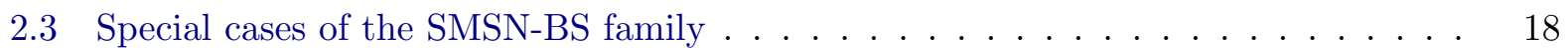

2.4 Maximum likelihood estimation . . . . . . . . . . . . . . . . . . . 22

2.4.1 The ML estimation based on the EM-algorithm . . . . . . . . . . . . . 23

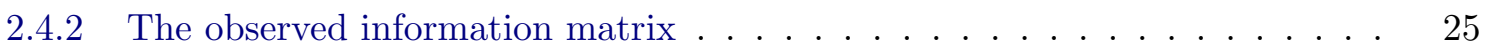

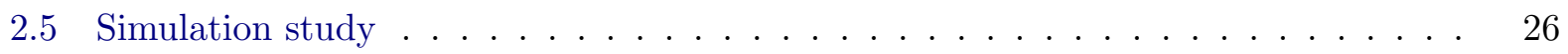

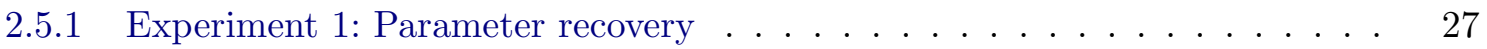

2.5.2 Experiment 2: Asymptotic properties . . . . . . . . . . . . 28

2.5.3 Experiment 3: Robustness properties of EM estimates . . . . . . . . . . 29

$2.6 \quad$ Breaking stress dataset . . . . . . . . . . . . . . . . . . . . 29

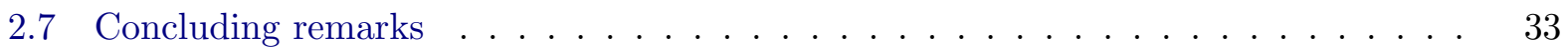

3 Sinh-skew-normal/Independent Regression Models 35

3.1 Introduction . . . . . . . . . . . . . . . . . . . . . . 35

3.2 The Sinh-SNI distribution . . . . . . . . . . . . . . . . . . . 37

3.2 .1 Special cases of the Sinh-SNI family . . . . . . . . . . . . . . 41

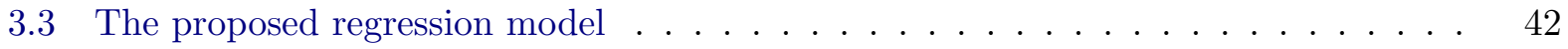

3.3.1 ML estimation using an EM-algorithm . . . . . . . . . . . . 43

3.3.2 The observed information matrix . . . . . . . . . . . . . . . 45

3.4 Simulation study . . . . . . . . . . . . . . . . . . . . . 47

3.4.1 Experiment 1: Recovery of parameters . . . . . . . . . . . 47 
3.4.2 Experiment 2: Asymptotic properties . . . . . . . . . . . . . . 52

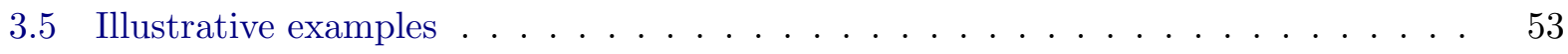

3.5.1 Biaxial fatigue dataset . . . . . . . . . . . . . . . . 53

3.5.2 Martin Marietta dataset . . . . . . . . . . . . . . . . 55

3.6 Concluding remarks $\ldots \ldots \ldots \ldots \ldots \ldots \ldots$

4 Influence analysis in Sinh-skew-normal/Independent regression models $\quad 61$

4.1 Introduction . . . . . . . . . . . . . . . . . . . . . . . . 62

4.2 The proposed regression model $\ldots \ldots \ldots \ldots \ldots \ldots$

4.3 Diagnostic analysis . . . . . . . . . . . . . . . . . . . 67

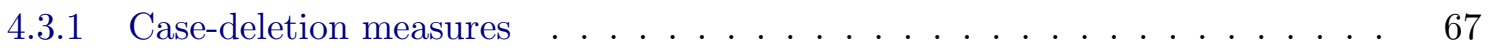

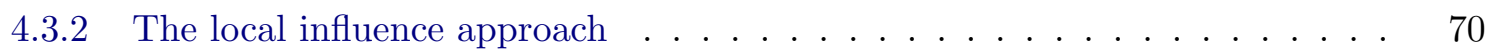

4.4 Numerical application . . . . . . . . . . . . . . . . . . . . . 73

4.4 .1 Simulation studies . . . . . . . . . . . . . . . . . 73

4.4 .2 Illustrative examples . . . . . . . . . . . . . . . . . . . 74

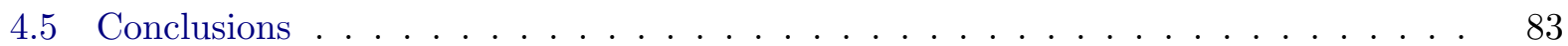

5 Conclusions $\quad 85$

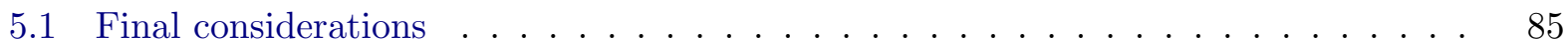

5.2 Suggestions for future research $\ldots \ldots \ldots \ldots \ldots$

$\begin{array}{ll}\text { A Special cases of sinh-SNI models } & 87\end{array}$

$\begin{array}{lr}\text { Bibliography } & 89\end{array}$ 


\section{List of Figures}

2.1 Density plots of: (a) BS, SN-BS and St-BS distributions, (b) SN-BS, ST-BS, SNCBS and SSL-BS distributions. (c) and (d) show left and right tails of the densities, all for $\lambda=3$.

2.2 Density plots of: (a) BS, SN-BS and St-BS distributions, (b) SN-BS, ST-BS, SNCBS and SSL-BS distributions. (c) and (d) show left and right tails of the densities,

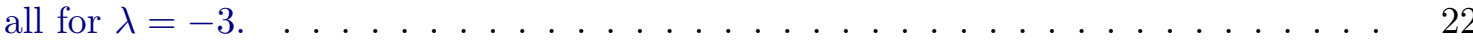

2.3 Bias (upper panel) and RMSE (lower panel) of parameters $\alpha, \beta$ and $\lambda$, respectively. 28

2.4 Average relative changes of estimates for different perturbations $\vartheta \quad \ldots \ldots$. . . . . 29

2.5 Stress. Histogram of the Stress data with five ML-fitted densities BS, SN-BS and SMSN-BS overlaid. . . . . . . . . . . . . . . . . . . 31

2.6 Stress. Histogram of the Stress data with five ML-fitted densities BS, SN-BS and SMN-BS overlaid. . . . . . . . . . . . . . . . . . . . 32

2.7 Stress. Q-Q plots and simulated envelopes. . . . . . . . . . . . . . . . 33

3.1 Density plots of the sinh-SNI distributions based on SN SCN, SSL and ST distributions for $\alpha=1$ (left) and $\alpha=5$ (right), respectively, when $\lambda=0,1,3 \ldots \ldots \ldots$.

3.2 Density plots of the sinh-SNI distributions based on SN, SCN, SSL and ST distributions for $\alpha=1$ (first line) and $\alpha=5$ (second line), respectively, when $\lambda=0,-1,-3 . \quad 42$

3.3 Bias of parameters $\alpha, \beta_{1}, \beta_{2}, \beta_{3}, \beta_{4}$, and $\lambda$ under sinh-SNI model. . . . . . . . 52

3.4 RMSE of MLEs of parameters $\alpha, \beta_{1}, \beta_{2}, \beta_{3}, \beta_{4}$, and $\lambda$ under sinh-SNI model. . . . 53

3.5 Biaxial dataset: Simulated envelopes for sinh-SNI-NLM based on SN, ST, SCN and

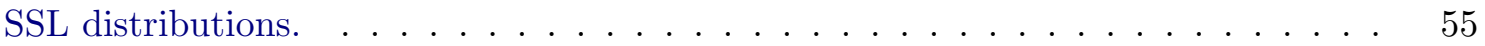

3.6 Biaxial dataset: Scatterplots and fitted lines based on sinh-SN-NLM and sinh-STNLM models. . . . . . . . . . . . . . . . . . . . . . . . . 55

3.7 Martin Marietta dataset: Scatterplots and fitted lines under the sinh-SN (left) and sinh-SCN (right) models. . . . . . . . . . . . . . . . 57

3.8 Martin Marietta dataset: Simulated envelopes under the four proposed models. . . 57

3.9 Martin Marietta dataset: Index plots of the distances $\left(d_{i}\right)$ for the fitted models. $\quad 58$

3.10 Martin Marietta dataset: Estimated $\kappa_{i}$ for the ST, SCN and SSL models. . . . . 59

4.1 Simulated data. Index plot of $M(0)$ in the case weights perturbation using sinh-SN

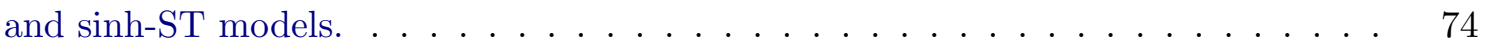

4.2 Biaxial dataset: Simulated envelopes for sinh-SNI-NLM based on SN, ST, SCN and SSL distributions. 
4.3 Biaxial dataset. Generalized Cook distance vs $Q$-distance (left) and $G D_{j}^{1}(\lambda)$ vs $G D_{j}^{1}(\alpha, \beta)$ (right). . . . . . . . . . . . . . . . . . . .

4.4 Biaxial dataset. Diagnostics of case weight perturbation (left), response variable perturbation (center) and work variable perturbation (right) (benchmark with $c^{*}=1$ ). 77

4.5 Biaxial dataset: $C_{i}$ for response perturbation (first line, (a)-(c)). $C_{i}$ for explanatory

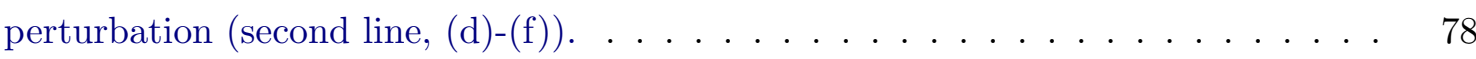

4.6 Martin Marietta dataset: Boxplots of the Mahalanobis distances for the four fitted models (left) and the estimated with $\kappa_{i}$ in the sinh-N, sinh-ST, sinh-SCN and sinhSSL models (right). . . . . . . . . . . . . . . . . . . . . .

4.7 Martin Marietta dataset: Index plot of generalized Cook distance (first line) and index plot of $Q$-distance (second line) using sinh-SN and sinh-ST models. . . . . .

4.8 Martin Marietta dataset: Index plot of $M(0)$ in the case weights perturbation (first line), response perturbation (second line) and explanatory perturbation (third line) in the sinh-SN and sinh-ST models. . . . . . . . . . . . . . . . . . . . . 


\section{List of Tables}

2.1 Means and MC-Sd of EM estimates based on 1000 samples, with IM-SE (the average value of the approximate standard error obtained through the information-based method) and Cov-MC (the coverage probability) from fitting the model. True values of parameters are in parentheses. . . . . . . . . . . . . . 27

2.2 Parameter estimates and SE of the SMSN-BS models and percentile estimates . .

2.3 Some information criteria (columns 2-4), log-likelihood (columns 5): SMSN-BS model vs corresponding SMN-BS model (in parentheses). . . . . . . . . . . . .

3.1 Model M1: Mean and MC Sd are the respective mean estimates and standard deviations under the sinh-ST linear model, while IM SE is the average value of the approximate standard error obtained through the information-based method. . . .

3.2 Model M2: Mean and MC Sd are the respective mean estimates and standard deviations under the sinh-SN and sinh-ST nonlinear models, while IM SE is the average value of the approximate standard error obtained through the information-based method. . . . . . . . . . . . . . . . . . . . . . .

3.3 Model M2: Mean and MC Sd are the respective mean estimates and standard deviations under the sinh-SSL and sinh-SCN nonlinear models, while IM SE is the average value of the approximate standard error obtained through the informationbased method. . . . . . . . . . . . . . . . . . . . . .

3.4 Biaxial dataset: ML estimates for the four selected sinh-SNI-NLMs, the log-likelihood values and the information criteria values, with sinh-NI-NLMs (in parentheses). . .

3.5 Martin Marietta dataset: Results from fitting four selected sinh-SNI models (information criteria values under sinh-NI model, in parentheses). . . . . . . . . . .

3.6 Martin Marietta dataset: Comparison of the relative changes in the ML estimates in term of TRC and MRC for the four selected sihn-SNI models. . . . . . . . . . .

4.1 Biaxial dataset: ML estimates for the four sinh-SNI-NLMs, the log-likelihood values and information criteria values (sinh-NI-NLMs in parentheses) . . . . . . . . 76

4.2 Biaxial dataset: Relative changes (\%) after dropping the cases indicated. . . . . . . 79

4.3 Martin Marietta dataset: Results from fitting with four selected sinh-SNI models (information criteria values in sinh-NI model in parentheses). . . . . . . . . . .

4.4 Martin Marietta dataset: Comparison of the relative changes in the ML estimates in terms of TRC and MRC for the four selected sinh-SNI models. . . . . . . . . . . 


\section{Chapter 1}

\section{Introduction}

The rupture or failure of materials is one of the major problems in the industrial area. One of the many causes of failure is fatigue, caused when the material is subjected to cyclical stress and tension producing the structural failure. The distribution proposed by Birnbaum and Saunders (1969a) has been used in the industrial area as a model of materials fatigue, which was inspired by vibration problems found in commercial aircraft. To solve this specific problem of fatigue, a family of distributions was constructed that allow modeling the lifetime of materials subject to dynamic stress.

Statistical models for fatigue processes allow describing the random variation of failure times associated with materials exposed to fatigue as a result of different patterns and cyclic forces. These materials can be characterized by parameters of lifetime distributions. Such characterizations are important to predict the performance of the materials subject to dynamic loads. Some probabilistic models that have been proposed to describe fatigue-life are, for example, the gamma, inverse gaussian (IG), lognormal and weibull distributions, which fit with great precision in the central region of the distribution. In situations where lifetimes are short or very long, such distributions are not appropriate, producing poor fit. Hence it is necessary to consider distributions with heavier tails, and/or distributions that allow better fit of short lifetimes, to obtain robust statistical results and, above all, to be easily implemented in practical situations.

Birnbaum and Saunders (1969b) originally obtained the maximum likelihood estimators of the parameters. (Mann et al., 1974, p. 155) showed that the Birnbaum-Saunders (BS) distribution is unimodal. Engelhardt et al. (1981) proposed confidence intervals for the parameters. In addition, they presented a hypothesis testing study. Desmond (1985) derived the BS distribution based on a biological model. The author also extended the physical justification for the use of this 
BS distribution, relaxing some assumptions made in Birnbaum and Saunders (1969a). Desmond (1986) investigated the relationship between the BS distribution and IG distribution. Achcar (1993) presented a Bayesian estimation method and Lu and Chang (1997) used bootstrap methods to construct prediction intervals for future observations of a BS distribution. Dupuis and Mills (1998) used robust estimation methods for BS distribution parameters in the presence of outliers.

The BS distribution arose in the context of materials engineering, but since it is appropriate to describe many processes of cumulative degradation, it has applications in other areas as well. Leiva et al. (2008a, 2009) presented some examples of applications (or bibliographical references) in health sciences, environment studies, forestry, demographics, actuary and finance, among others. All these aspects have allowed considering the BS distribution as a probability model instead of a model that only allows modeling lifetimes; see Marshall and Olkin (2007). In the areas mentioned above, one finds covariates that normally explain adequately the failure time. Rieck and Nedelman (1991) formulated and developed a log-linear model for the BS distribution, which in this case is based on the Sinh-Normal distribution (Rieck, 1989).

Some generalizations and applications of the BS distribution were considered introducing new parameters, or considering symmetric/asymmetric distributions instead of normal distribution, in order to obtain more robust or more flexible models to be used in real problems where atypical observations are present. For example, Owen and Padgett (2000) developed the three-parameter distribution. Barros et al. (2008) considered the Student- $t$ distribution in place of the standard normal distribution, where the degrees of freedom allow controlling the kurtosis of the resulting distribution, producing a robust extension of the usual BS distribution. Based on this extension, Barros et al. (2008) presented an inference and diagnostic study (based on the method of Cook, 1986), an extension of some results obtained by Galea et al. (2004). Díaz-García and Leiva (2005) generalized the BS distribution based on the class of elliptical distributions, which includes the Cauchy, Laplace, logistic, normal and Student-t distributions, among others. Barros et al. (2009) developed a package called gbs (included in the R software) for the generalized BS distribution, which can be obtained in CRAN (http://CRAN.R-project.org/), version 1.0. An extension of the BS distribution based on scale mixture of normal was developed by Balakrishnan et al. (2009).

Finally, instead of considering a symmetric distribution to obtain a generalization of the BS distribution, an asymmetric distribution, the skew elliptical, can be considered. Some results can be found in Vilca and Leiva (2006), Leiva et al. (2010), Vilca et al. (2011) and Santana et al. (2011). 


\subsubsection{Birnbaum-Saunders model}

Let $T$ be a random variable (r.v.) which represents the total time until failure occurs. Birnbaum and Saunders (1969a) showed that under certain conditions, the cumulative distribution function (cdf) of $T$ is given by

$$
F_{T}(t)=\Phi\left(\frac{1}{\alpha}\left(\sqrt{\frac{t}{\beta}}-\sqrt{\frac{\beta}{t}}\right)\right), t>0, \alpha>0, \beta>0
$$

where $\Phi(\cdot)$ is the cdf of the standard normal distribution. In this case, we say that $T$ follows a BS distribution with parameters $\alpha$ and $\beta$. We denote this distribution by $T \sim B S(\alpha, \beta)$. It can be shown that the probability density function (pdf) of $T$ is given by

$$
f_{T}(t)=\phi\left(a_{t}(\alpha, \beta)\right) A_{t}(\alpha, \beta), \quad t>0
$$

where $\phi($.$) is the pdf of a N(0,1)$,

$$
a_{t}(\alpha, \beta)=\frac{1}{\alpha}\left(\sqrt{\frac{t}{\beta}}-\sqrt{\frac{\beta}{t}}\right) \text { e } A_{t}(\alpha, \beta)=\frac{d}{d t} a_{t}(\alpha, \beta) .
$$

An important property in the construction of this distribution is that a r.v.

$$
Z=\frac{1}{\alpha}\left(\sqrt{\frac{T}{\beta}}-\sqrt{\frac{\beta}{T}}\right)
$$

follows a $N(0,1)$ distribution. The above expression is equivalent to saying that a r.v. $T$ defined by

$$
T=\frac{\beta}{4}\left[\alpha Z+\sqrt{\alpha^{2} Z^{2}+4}\right]^{2}
$$

follows a $B S(\alpha, \beta)$ distribution. Some extensions of the BS distribution are based essentially on assigning a certain distribution to $Z$ given in equation (1.3), for example Díaz-García and Leiva (2005) presented a theoretical study of the BS distribution under the class of elliptical distributions, providing lifetime distributions that grow faster and have tails with less or greater kurtosis than the usual BS distribution. Based on a similar procedure, Vilca and Leiva (2006) obtained a broader generalization by developing the BS distribution under the class of skew-elliptical distributions, which has the property of being more flexible regarding kurtosis and asymmetry.

Note that the normal distribution and the Student- $t$ distribution are particular members of the class of (symmetric) scale mixture of normal (SMN) distributions; see Andrews and Mallows (1974), and 
Lange and Sinsheimer (1993). Some advantages of working with SMN distributions are for example;

i) The possibility of developing estimation algorithms, so that the computational implementation becomes less difficult, consequently allowing successful application in many situations where the normal distribution is not adequate (especially in the presence of atypical observations in the data);

ii) The BS distribution based on the scale mixture of normal, which contains the usual BS distribution, the BS distribution based on the contaminated normal, the BS distribution based on the Laplace and the BS distribution based on the Slash (see Balakrishnan et al., 2009). The BS distribution based on the scale mixture of normal allows modeling the lowest and/or highest percentiles of the distribution in the presence of atypical observations (see, Balakrishnan et al., 2009);

iii) The application of methods of diagnostics and local influence, which are simpler under the class of SMN distributions. In this case, the method of Zhu and Lee (2001) and Zhu et al. (2001) instead of the method of Cook (1986). The methods of Zhu and Lee (2001) and Zhu et al. (2001) have also been successfully applied in models based on the class of scale mixture of skew-normal distributions, see for example Matos et al. (2013) and Zeller et al. (2010).

\subsubsection{The scale mixtures of skew-normal distribution}

When the data distribution has asymmetric behavior this can be modeled through the class of distributions called asymmetric distributions, see Branco and Dey (2001). Within this class, the univariate skew-normal distribution is perhaps the pioneer in this idea, initially introduced by O'Hagan and Leonard (1976) as an a priori distribution in Bayesian analysis and later formally studied by Azzalini (1985) (from the classic point of view) as a natural extension of the normal distribution, often used to model the asymmetric structure present in the data. Using this idea, other asymmetric distributions such as skew-t, skew-Cauchy, skew-slash, and skew-power exponential were developed to model asymmetric data in the presence of atypical observations. The inference study under the class of asymmetric distributions or statistical models based on skew normal have been applied successfully in both theoretical and practical contexts.

According to Azzalini (1985), a r.v. $Z$ has a skew-normal distribution, if its pdf is given by

$$
f(z)=2 \phi\left(z ; \mu, \sigma^{2}\right) \Phi\left(\frac{\lambda}{\sigma}(z-\mu)\right), \quad z \in \mathbb{R}
$$


where $\phi\left(. ; \mu, \sigma^{2}\right)$ is the pdf of the $N\left(\mu, \sigma^{2}\right)$ distribution, and $\Phi($.$) is the cdf of the N(0,1)$. This distribution is usually denoted by $Z \sim S N\left(\mu, \sigma^{2}, \lambda\right)$, where $\lambda \in \mathbb{R}$ regulates the asymmetry of the distribution. For $\lambda=0$, we have that $Z \sim N\left(\mu, \sigma^{2}\right)$. The stochastic representation of $Z$ is given by

$$
Z \stackrel{d}{=} \mu+\sigma\left[\delta\left|X_{0}\right|+\left(1-\delta^{2}\right)^{1 / 2} X_{1}\right], \quad \text { com } \quad \delta=\frac{\lambda}{\sqrt{1+\lambda^{2}}}
$$

where $X_{0} \sim N_{1}(0,1)$ and $X_{1} \sim \mathrm{N}(0,1)$ are independent and $\left|X_{0}\right|$ denotes the absolute value of $X_{0}$. The above stochastic representation is important, for example, to obtain the maximum likelihood estimate (MLE) based on the EM algorithm.

The asymmetric class of distributions of scale mixture of skew-normal (SMSN) is attractive due to the fact that it includes the symmetric and asymmetric versions of distributions, Student- $t$, slash, power exponential and contaminated normal, among others, all with heavier tails than the normal distribution. A random variable $Z$ follows a SMSN distribution with location parameters $\mu \in \mathbb{R}$, dispersion parameter $\sigma>0$ and additional parameter $\boldsymbol{\nu}$ if its pdf is given by

$$
\phi_{\mathrm{SMSN}}(z)=2 \int_{0}^{\infty} \phi\left(z ; \mu, \kappa(u) \sigma^{2}\right) \Phi\left(\lambda \kappa^{-1 / 2}(u) \frac{z-\mu}{\sigma}\right) d G(u ; \boldsymbol{\nu})
$$

where $G(. ; \boldsymbol{\nu})$ is the cdf of the positive random variable $U, \Phi($.$) is the cdf of the N(0,1)$, and $\kappa($. is a positive function of $U$. The above distribution will be denoted by $Z \sim \operatorname{SMSN}\left(\mu, \sigma^{2}, \lambda ; G\right)$. When $\kappa(U)=1$ with probability 1, we obtain the known skew-normal distribution. Now, if in (2.4) $\lambda=0$, then we obtain the scale mixture of normal (SMN) distribution, some results can be found in Andrews and Mallows (1974), Dempster et al. (1980) and Lange and Sinsheimer (1993). In this case the pdf is given by

$$
\phi_{\mathrm{MSN}}(z)=\int_{0}^{\infty} \phi\left(z ; \mu, \kappa(u) \sigma^{2}\right) d G(u ; \boldsymbol{\nu})
$$

The stochastic representation corresponding to the distribution given in (1.7) is given by

$$
Z=\mu+[\kappa(U)]^{\frac{1}{2}} Z_{0}
$$

where $Z_{0} \sim \mathrm{SN}\left(0, \sigma^{2}, \lambda\right)$ and $U$ is a positive random variable with cdf $G$ independent of $Z_{0}$. The stochastic representation given above can be used for the implementation of the EM algorithm, to obtain MLE. The Mahalanobis distance, $d_{\lambda}^{2}=(Z-\mu)^{2} / \sigma^{2}$ can be used to fit the model, see Lachos et al. (2010b). Some examples of scale mixture of skew-normal distributions are described below: 


\section{Examples of scale mixture of skew-normal distributions}

- Skew student-t distribution with $\nu>0$ degrees of freedom $S T\left(\mu, \sigma^{2} ; \lambda, \nu\right)$. In this case $U \sim$ $\operatorname{Gamma}(\nu / 2, \nu / 2)$, see for example Gupta (2003). For the symmetric case, see for example Little (1988) and Lange et al. (1989).

$$
f(z)=2 t_{1}(z ; \mu, \sigma, \nu) T_{1}\left(\frac{\sqrt{\nu+1}}{\sqrt{d+1}} \frac{\lambda(z-\mu)}{\sigma} \mid 0,1, \nu+1\right), \quad z \in \mathbb{R} .
$$

where $t_{1}(\cdot ; \mu, \sigma, \nu)$ denotes the pdf of the Student-t distribution and $T_{1}(\cdot \mid 0,1, \nu)$ denotes the cdf of the standard Student-t distribution.

- Skew slash distribution, $Z \sim S S L\left(\mu, \sigma^{2}, \lambda ; \nu\right)$, with shape parameter $\nu>0$.

In this case $U \sim \operatorname{Beta}(\nu, 1)$. This is the pdf is given by $h(u ; \nu)=\nu u^{\nu-1} \mathbb{I}_{(0,1)}$. So we will have:

$$
f(z)=2 \nu \int_{0}^{1} u^{\nu-1} \phi\left(z ; \mu, \frac{\sigma^{2}}{u}\right) \Phi\left(u^{1 / 2} \lambda \sigma^{-1}(z-\mu)\right), \quad z \in \mathbb{R}^{p} .
$$

The skew-normal distribution is obtained when $\nu \uparrow \infty$, see Wang and Genton (2006).

- Skew normal contaminated distribution, $Z \sim S C N\left(\mu, \sigma^{2}, \lambda ; \nu, \gamma\right), 0 \leq \nu \leq 1,0<\gamma \leq 1$ (Little (1988); Yamaguchi (1990)). The pdf of $U$ is given by

$$
h(u ; \boldsymbol{\nu})=\nu \mathbb{I}_{\{\gamma\}}(u)+(1-\nu) \mathbb{I}_{\{1\}}(u), \quad \boldsymbol{\nu}=(\nu, \gamma)^{\top},
$$

where the notation $\mathbb{I}_{(A)}$ is the indicator function of the set $A$. Thus the pdf of $Z$ is given by

$$
f(z)=2\left\{\nu \phi\left(z ; \mu, \frac{\sigma^{2}}{\gamma}\right) \Phi\left(\gamma^{1 / 2} \frac{\lambda}{\sigma}(z-\mu)\right)+(1-\nu) \phi\left(z ; \mu, \sigma^{2}\right) \Phi\left(\frac{\lambda}{\sigma}(z-\mu)\right)\right\}(1.1
$$

In this case, the skew-normal is obtained when $\nu=0$.

\subsubsection{Local influence}

In practical situations where we have a set of related data, for example with lifetime, it is of first interest to perform a descriptive analysis of the data in order to observe if any particular observation controls important properties in the estimation of the parameters, and in general in the statistical analysis. Thus, an important step is to try to detect atypical observations and evaluate the impact of the exclusion of the observations on the estimation of the parameters. Some results on this subject in the context of regression models can be found in Cook and Weisberg (1982) and 
Chatterjee and Hadi (1988).

A more general notion to evaluate the influence of certain observations under a certain perturbation scheme was proposed by Cook (1986). This method is known in the literature as the local influence method, which allows evaluating the influence of observations, under small perturbations in some components of the model, on the estimation of parameters, and other results of the analysis. This diagnostic technique has been studied by many authors, for example, Thomas and Cook (1990), who presented a study of local influence in generalized linear model, Lesaffre and Verbeke (1998)), who considered the local influence in the mixed linear model, Zhao and Lee (1998), who applied the local influence method in the model of simultaneous equations, and Galea et al. (1997), who applied the method in the elliptical linear regression model. Galea et al. (2005) present a diagnostic study in the comparative calibration model, considering that the data follow a Student- $t$ distribution. Another method of local influence detection that has been recently applied was proposed by Zhu and Lee (2001). The methodologies mentioned are described as follows:

\section{Cook method}

For a set of observations, let $\ell(\boldsymbol{\theta})$ be the log-likelihood function corresponding to the postulated model. We introduce perturbations in the model through a $\boldsymbol{\omega}: q \times 1$ vector in some open subset of $\mathbb{R}^{q}$. Assume that the perturbation scheme is well defined. We denote by $\ell(\boldsymbol{\theta} \mid \boldsymbol{\omega})$ the log-likelihood function corresponding to the disturbed model and assume that there is a $\boldsymbol{\omega}_{0}$ such that $\ell\left(\boldsymbol{\theta} \mid \boldsymbol{\omega}_{0}\right)=$ $\ell(\boldsymbol{\theta})$, for all $\boldsymbol{\theta}$. To evaluate the influence of the perturbation $\boldsymbol{\omega}$ on the MLE $\widehat{\boldsymbol{\theta}}$, Cook (1986) suggested studying the behavior of the likelihood distance, defined by

$$
L D(\boldsymbol{\omega})=2\left[\ell(\widehat{\boldsymbol{\theta}})-\ell\left(\widehat{\boldsymbol{\theta}}_{\omega}\right)\right]
$$

around $\boldsymbol{\omega}=\boldsymbol{\omega}_{0}$, where $\widehat{\boldsymbol{\theta}}_{\omega}$ denotes the maximum likelihood estimate under $\ell(\boldsymbol{\theta} \mid \boldsymbol{\omega})$. From this perspective, the graph of $L D(\boldsymbol{\omega})$ contains essential information about the influence of the assumed perturbation scheme. The idea is to analyze how the surface $\alpha(\boldsymbol{\omega})=\left(\boldsymbol{\omega}^{\top}, L D(\boldsymbol{\omega})\right)^{\top}$ deviates from its tangent plane in $\boldsymbol{\omega}_{0}$. This analysis can be done by studying the curvatures of the normal sections on the surface $\alpha(\boldsymbol{\omega})$ in $\boldsymbol{\omega}_{0}$, which are intersections of $\alpha(\boldsymbol{\omega})$ with planes containing the normal vector to its tangent plane in $\boldsymbol{\omega}_{0}$. Cook (1986) showed that the normal curvature in the unitary direction $\mathbf{d}$ is given by

$$
C_{d}=2\left|\mathbf{d}^{\top} \boldsymbol{\Delta}^{\top} \ddot{\mathbf{I}}^{-1} \boldsymbol{\Delta} \mathbf{d}\right|, \text { com }\|\mathbf{d}\|=1,
$$


where $-\ddot{\mathbf{I}}$ is the observed information matrix under the postulated model and $\boldsymbol{\Delta}$ is the matrix of second partial derivatives with respect to $\boldsymbol{\theta}$ and $\boldsymbol{\omega}$, evaluated an $\boldsymbol{\theta}=\widehat{\boldsymbol{\theta}}$ and $\boldsymbol{\omega}=\boldsymbol{\omega}_{0}$. The curvature $C_{d}$ can be used to evaluate the influence of the perturbation scheme on the components of the model. According to Cook (1986), the direction that produces the greatest local change in the parameter estimation is given by $\boldsymbol{d}_{\text {max }}$ which corresponds to the eigenvector associated with the largest important eigenvalue of $\boldsymbol{\Delta}^{\top} \ddot{\mathbf{I}}^{-1} \boldsymbol{\Delta}$ and is used to identify observations that may control important properties in data analysis.

\section{Zhu and Lee method}

For incomplete data Zhu and Lee (2001) proposed an alternative method to perform diagnostic studies considering a type of likelihood distance defined by

$$
f_{Q}(\boldsymbol{w})=2\left(Q(\widehat{\boldsymbol{\theta}} \mid \widehat{\boldsymbol{\theta}})-Q\left(\widehat{\boldsymbol{\theta}}_{w} \mid \widehat{\boldsymbol{\theta}}\right)\right),
$$

where $\left.Q(\boldsymbol{\theta} \mid \widehat{\boldsymbol{\theta}})=E\left[\ell\left(\boldsymbol{\theta}, \boldsymbol{Y}_{c}\right) \mid \boldsymbol{Y}, \widehat{\boldsymbol{\theta}}\right)\right]$, and $\boldsymbol{Y}_{c}$ is the vector composed of the observations $\boldsymbol{Y}$ and the unobserved data. Similar to the method of Cook (1986), Zhu and Lee (2001) studied the behavior of the surface $\boldsymbol{\alpha}(\boldsymbol{\omega})=\left(\boldsymbol{\omega}^{\top}, f_{Q}(\boldsymbol{\omega})\right)^{\top}$, through the normal curvature $C_{f_{Q}, \mathbf{d}}$ in the direction of a unit vector $\mathbf{d}$, which can be used to represent the behavior of the function $f_{Q}(\boldsymbol{w})$

$$
C_{f_{Q}, \mathbf{d}}=-2 \mathbf{d}^{\top} \ddot{Q} \boldsymbol{w}_{o} \mathbf{d}, \quad-\ddot{Q}_{\boldsymbol{\omega}_{0}}=\Delta_{\boldsymbol{\omega}_{o}}^{\top}\left\{-\ddot{Q}_{\theta}(\widehat{\boldsymbol{\theta}})\right\}^{-1} \Delta_{\boldsymbol{\omega}_{0}}
$$

where $\ddot{Q}_{\boldsymbol{\theta}}(\widehat{\boldsymbol{\theta}})=\left.\frac{\partial^{2} Q(\boldsymbol{\theta} \mid \widehat{\boldsymbol{\theta}})}{\partial \boldsymbol{\theta} \partial \boldsymbol{\theta}^{\top}}\right|_{\boldsymbol{\theta}=\widehat{\boldsymbol{\theta}}}$ e $\Delta_{\boldsymbol{\omega}}=\left.\frac{\partial^{2} Q(\boldsymbol{\theta}, \boldsymbol{\omega} \mid \widehat{\boldsymbol{\theta}})}{\partial \boldsymbol{\theta} \partial \boldsymbol{\omega}^{\top}}\right|_{\boldsymbol{\theta}=\widehat{\boldsymbol{\theta}}(\boldsymbol{\omega})}$. As in Cook (1986), the expression $-\ddot{Q} \boldsymbol{\omega}_{0}$ is fundamental to identify atypical observations.

\subsection{Thesis organization}

This thesis consists of three papers. The first paper describes the BS distribution family based on the scale mixture of skew-normal (SMSN-BS), highlighting its properties, stochastic representation, as well as some special cases of distributions belonging to the family. Then, an estimation process is approached using the EM algorithm, which uses the complete likelihood of the SMSN-BS distribution and soon after it is shown how it is possible to approximate the observed information matrix. Three simulation studies are performed to show the performance of the EM algorithm in the sense of parameter estimation, asymptotic properties and robustness. Finally, an application of the model is performed, considering the dataset about the breaking stress of carbon fibers, pre- 
viously analyzed Nichols and Padgett (2006) and Cordeiro et al. (2013).

Then, the second article presents the family of sinh-skew-normal/independent (sinh-SNI) distributions, which allow applications in the regression model. Properties of the sinh-SNI distribution are studied, such as the stochastic representation and the moment generating function. It presents some special cases of distributions that are part of the family. Next, the sinh-SNI regression model is discussed. Conditional distributions for the EM algorithm are developed for the estimation of model parameters. Subsequently, the observed information matrix is approximated. In order to show the performance of the EM algorithm, two simulation studies are carried out to evaluate the estimation of parameters and good asymptotic properties. Also two applications using the fatigue data and the Martin Marietta company data are developed to fit a nonlinear and linear regression model, respectively.

Finally, in the third paper, a global and local influence diagnostic study is presented, using the method developed by Zhu and Lee (2001), using the complete likelihood. The Hessian matrix is calculated and the perturbations of case weight, response and explanatory variables are analyzed. A simulation study using the sinh-SNI regression model is conducted, under the perturbation of some point to verify the model's ability to detect influential points. Also, two applications using the diagnostic technique in the fatigue data and the Martin Marietta company data are developed, to fit the nonlinear and linear regression model, respectively.

Suggestions for future studies and some conclusions are discussed in Chapter 5. 


\title{
Chapter 2
}

\section{Modeling extreme percentiles in}

\section{Birnbaum-Saunders distributions}

\section{based on an asymmetric heavy-tailed}

\section{distribution}

\begin{abstract}
The use of the Birnbaum-Saunders (BS) distribution has recently received considerable attention in the statistical literature, both in applied and theoretical problems. Even though much work has been done on extensions of the BS distribution, these are still inadequate for predicting extreme percentiles and fitting data that are highly concentrated on the left-tail of the distribution. Vilca et al. (2011) proposed a BS distribution based on the skew-normal distribution that is able to fit the extreme lower percentiles very well. We propose here a robust extension of the BS distribution, based on scale mixtures of skew-normal distributions that can be used to model asymmetric data. This extension provides flexible thick-tailed distributions which can be used in robust estimation of parameters in the presence of outlying observations, as well as the implementation of an EM algorithm for maximum likelihood estimation of the model parameters. Finally, results of a simulation study are presented along with an application to a real dataset.
\end{abstract}

Keywords Birnbaum-Saunders distribution; Skew-normal distribution; EM-algorithm; Scale mixtures of skew-normal distributions. 


\subsection{Introduction}

During the last two decades, considerable attention has been paid to the construction of classes of flexible parametric distributions that can be used in problems that arise in diverse practical applications. As an example, the normal distribution has been extended to the flexible classes of symmetric and asymmetric distributions; see Genton (2004).

Birnbaum and Saunders (1969a) developed an important life distribution from a problem of material fatigue, the Birnbaum-Saunders (BS) distribution, which possesses many attractive features and properties. This distribution has found many important applications in several fields, like as engineering, industry, business, reliability, survival analysis, and medical sciences. For example, Desmond (1985) considered the BS distribution as a biological model, Leiva et al. (2007) and Barros et al. (2008) presented an application in the medical field, and Podlaski (2008), Leiva et al. (2008a, 2009, 2010), and Vilca et al. (2010) used this model to describe some data from forestry and environmental sciences. Owen and Padgett (2000) presented a discussion on advantages the use of BS distribution in the estimation of percentiles that are of interest to engineers.

A positive random variable $T$ is said to have a BS distribution if it is related to the normal distribution through of the representations $T=(\beta / 4)\left[\alpha Z_{0}+\sqrt{\alpha^{2} Z_{0}^{2}+4}\right]^{2}$, where $Z_{0} \sim N(0,1)$ and the probability density function (pdf) of $T$ is

$$
f_{T}(t)=\phi\left(a_{t}(\alpha, \beta)\right) A_{t}(\alpha, \beta), t>0
$$

where $\phi($.$) denotes the pdf of N(0,1)$ distribution, $a_{t}(\alpha, \beta)=(1 / \alpha)(\sqrt{t / \beta}-\sqrt{\beta / t})$ and $A_{t}(\alpha, \beta)$ is the derivative of $a_{t}(\alpha, \beta)$ with respect to $t$. The usual notation is $T \sim \mathrm{BS}(\alpha, \beta)$.

Some generalizations of the BS distribution have been proposed; see for example Díaz-García and Leiva (2005), Leiva et al. (2008b), Gomez et al. (2009), and Balakrishnan et al. (2009). The last work developed a BS distribution based on scale mixture of normal (SMN) distributions. Even though some of these generalizations have been applied with success, mainly due to the robust parameter estimation, these models are still inadequate to fit data that are highly concentrated on the left-tail of the distribution, and may not be able to predict extreme percentiles properly. A first step to overcome this problem was given by Vilca et al. (2011) who considered the skew-normal distribution in place of the normal one, resulting in the skew-normal Birnbaum-Saunders (SN-BS) distribution. It allows predicting extreme percentiles very well, especially on the left-tail. These extreme lower percentiles are not very well estimated employing the usual BS models. In fact, if 
the distribution of data is highly concentrated on the left-tail the usual BS model overestimates the lower percentiles. Moreover, if the data contain outlying observations the SN-BS distribution of Vilca et al. (2011) may not be suitable to represent data containing outlying observations due to its close dependence on the skew-normal distribution. To overcome this problem, we exploit here the relationship between the SN-BS and skew-normal distributions to obtain a general class of BS distributions based on the family of scale mixture skew-normal (SMSN) distributions. The resulting distribution will be called the scale mixture of skew-normal Birnbaum-Saunders (SMSNBS) distribution.

\subsubsection{Motivating Examples}

Before discussing the goal of this work, we present motivating examples.

(i) Let $T_{1}$ and $T_{2}$ be two independent random variables such as $T_{j} \sim \mathrm{BS}(\alpha, \beta)$, for $j=1,2$. Then, the probability density functions (pdf) of $W_{1}=\min \left\{T_{1}, T_{2}\right\}$ and $W_{2}=\max \left\{T_{1}, T_{2}\right\}$ can be expressed as

$$
f_{W_{j}}(t)=2 \phi\left(a_{t}(\alpha, \beta)\right) \Phi\left(\lambda_{j} a_{t}(\alpha, \beta)\right) A_{t}(\alpha, \beta), t>0
$$

where $\lambda_{1}=-1, \lambda_{2}=1$, and $\phi($.$) and \Phi($.$) denote the pdf and the cumulative distribution$ function (cdf) of the standard normal distribution, respectively. This example motived Vilca et al. (2011) to consider the BS distribution based on the skew-normal distribution of Azzalini (1985), called the skew-normal Birnbaum-Saunders (SN-BS) distribution. A random variable $Z_{0}$ is said to have a skew-normal distribution if its pdf is given by $f_{0}(z)=2 \phi(z) \Phi(\lambda z)$, where $\lambda$ is the skewness parameter, and is denoted by $Z_{0} \sim \mathrm{SN}(0,1, \lambda)$, which has stochastic representation given by

$$
Z_{0}=\delta\left|X_{0}\right|+\left(1-\delta^{2}\right)^{1 / 2} X_{1}
$$

where $\delta=\lambda / \sqrt{1+\lambda^{2}}$, and $X_{0} \sim N(0,1)$ is independent of $X_{1} \sim N(0,1)$.

(ii) A bivariate random vector $\mathbf{T}=\left(T_{1}, T_{2}\right)^{\top}$ is said to have a bivariate Birnbaum-Saunders distribution if its cdf is of the form (see Kundu et al. (2010))

$$
F_{\mathbf{T}}(\mathbf{t})=\Phi_{2}\left(a_{\mathbf{t}}(\boldsymbol{\alpha}, \boldsymbol{\beta}) ; \rho\right)
$$

where $\Phi_{2}(. ; \rho)$ is the cdf of the distribution $\mathrm{N}_{2}(\mathbf{0}, \boldsymbol{\Sigma})$, with $\boldsymbol{\Sigma}$ being a $2 \times 2$ positive-definite 
correlation matrix, $\boldsymbol{\alpha}=\left(\alpha_{1}, \alpha_{2}\right)^{\top}$ and $\boldsymbol{\beta}=\left(\beta_{1}, \beta_{2}\right)^{\top}$ in $\mathbb{R}_{+}^{2}$, which is the positive part of $\mathbb{R}^{2}$, $-1<\rho<1$ and $a_{\mathbf{t}}(\boldsymbol{\alpha}, \boldsymbol{\beta})=\left(a_{t_{1}}\left(\alpha_{1}, \beta_{1}\right), a_{t_{2}}\left(\alpha_{2}, \beta_{2}\right)\right)^{\top}$, with $a_{t_{j}}\left(\alpha_{j}, \beta_{j}\right)=\left(1 / \alpha_{j}\right)\left(\sqrt{t_{j} / \beta_{j}}-\right.$ $\left.\sqrt{\beta_{j} / t_{j}}\right), j=1,2$. Now, suppose we are interested in the conditional distribution of $T_{2}$, given $T_{1}>t_{1}$ which is given by

$$
f_{T_{2} \mid T_{1}}\left(t_{2} \mid T_{1}>t_{1}\right)=\frac{\phi\left(a_{t_{2}}\left(\alpha_{2}, \beta_{2}\right)\right) A_{t_{2}}\left(\alpha_{2}, \beta_{2}\right)}{1-\Phi\left(a_{t_{1}}\left(\alpha_{1}, \beta_{1}\right)\right)} \Phi\left(\lambda a_{t_{2}}\left(\alpha_{2}, \beta_{2}\right)-\lambda_{\rho} a_{t_{1}}\left(\alpha_{1}, \beta_{1}\right)\right),
$$

where $\lambda=\rho / \sqrt{1-\rho^{2}}$ and $\lambda_{\rho}=1 / \sqrt{1-\rho^{2}}$. This pdf corresponds to a type of BS distribution based on a skew-normal distribution. For the special case $t_{1}=\beta_{1}$, we have

$$
f_{T_{2} \mid T_{1}}\left(t_{2} \mid T_{1}>\beta_{1}\right)=2 \phi\left(a_{t_{2}}\left(\alpha_{2}, \beta_{2}\right)\right) A_{t_{2}}\left(\alpha_{2}, \beta_{2}\right) \Phi\left(\lambda a_{t_{2}}\left(\alpha_{2}, \beta_{2}\right)\right) .
$$

This result just corresponds to the BS distribution based on the skew-normal distribution of Azzalini (1985), the SN-BS distribution discussed by Vilca et al. (2011).

The rest of this paper is organized as follows. In Section 2.2, we define the BS distribution based on SMSN distributions and discuss some of its properties. Specifically, we derive its pdf, conditional and marginal distributions. In Section 2.3, we present expressions for the pdfs and key properties of some special cases of the proposed model. In Section 2.4, we present the EM algorithm for maximum likelihood (ML) estimation along with the observed information matrix. In Section 2.6, we provide an illustrative example that displays the usefulness of the generalized BS distributions for fitting a real dataset that has been analyzed before in the literature. Finally, some concluding remarks are made in Section 2.7.

\subsection{The SMSN-BS distribution}

Before defining the proposed model, we present a brief introduction of SMSN distributions proposed in Branco and Dey (2001), in which a random variable $Z$ defined through the relation $Z=\mu+\sigma \kappa^{1 / 2}(U) Z_{0}$, where $Z_{0} \sim \mathrm{SN}(0,1, \lambda)$ and $\kappa(U)>0$, function of the positive mixing variable $U$ independent of $Z_{0}$, which has its cumulative distribution function (cdf) $G(. ; \boldsymbol{\nu})$, where $\boldsymbol{\nu}$ is a scalar or vector indexing the distribution of $U$. The pdf of $Z$ is given by

$$
\phi_{\operatorname{SMSN}}(z)=2 \int_{0}^{\infty} \phi\left(z ; \mu, \kappa(u) \sigma^{2}\right) \Phi\left(\lambda \kappa^{-1 / 2}(u)\left(\frac{z-\mu}{\sigma}\right)\right) d G(u ; \boldsymbol{\nu})
$$


We use the notation $Z \sim \operatorname{SMSN}\left(\mu, \sigma^{2}, \lambda ; G\right)$. When $\lambda=0$, the SMSN distribution reduces to the SMN distribution (Lange and Sinsheimer, 1993) and it is denoted by $Z \sim \operatorname{SMN}\left(\mu, \sigma^{2} ; G\right)$. If $\mu=0$ and $\sigma=1$, we have the standard SMSN distribution and it is denoted by $Z \sim \operatorname{SMSN}(0,1, \lambda ; G)$.

Now, following the same line as above and inspired by the works of Vilca et al. (2011) and Balakrishnan et al. (2009), a positive random variable $T$ is said to have a SMSN-BS distribution with parameter $\alpha>0, \beta>0$ and $\lambda \in \mathbb{R}$ if it can be represented by

$$
T=\frac{\beta}{4}\left[\alpha \kappa^{1 / 2}(U) Z_{0}+\sqrt{\alpha^{2} \kappa(U) Z_{0}^{2}+4}\right]^{2}
$$

where $Z_{0} \sim \operatorname{SN}(0,1 ; \lambda)$, which can be represented by $Z_{0}=\delta H+X_{1} \sqrt{1-\delta^{2}}$, where $H=\left|X_{0}\right|$ and $Z_{1}$ are independent, and they have half-normal and normal distributions, respectively. This distribution of $T$ is denoted by $T \sim \operatorname{SMSN}-\mathrm{BS}(\alpha, \beta, \lambda ; G)$. When $\lambda=0$, the SMSN-BS distribution reduces to the SMN-BS distribution of Balakrishnan et al. (2009). Moreover, the SN-BS distribution (Vilca et al. (2011)) is a special case when $\mathrm{G}$ is degenerate $(\kappa(u)=1)$. As the usual BS distribution, we also have that $Z=\kappa^{1 / 2}(U) Z_{0}$ can be expressed in terms of $T$ by

$$
Z=a_{T}(\alpha, \beta)=\frac{1}{\alpha}[\sqrt{T / \beta}-\sqrt{\beta / T}] \sim \operatorname{SMSN}(0,1, \lambda ; G)
$$

One can easily see from (2.5) that the conditional distribution of $T$, given $U=u$, is the $\operatorname{SN}-\operatorname{BS}\left(\alpha \kappa^{1 / 2}(u), \beta, \lambda\right)$ distribution. This result is used to derive the closed-form density of $T$, which is presented in the next theorem following the ideas of Balakrishnan et al. (2009) and Vilca et al. (2011).

Theorem 1. Let $T \sim S M S N-B S(\alpha, \beta, \lambda ; G)$. Then, the pdf of $T$ can be expressed as

$$
\begin{aligned}
f_{T}(t) & =\phi_{\mathrm{SMSN}}\left(a_{t}(\alpha, \beta)\right) A_{t}(\alpha, \beta), t>0 \\
& =2 \int_{0}^{\infty} \phi\left(a_{t}(\alpha, \beta) ; 0, \kappa(u)\right) \Phi\left(\lambda \kappa^{-1 / 2}(u) a_{t}(\alpha, \beta)\right) A_{t}(\alpha, \beta) d G(u ; \boldsymbol{\nu}),
\end{aligned}
$$

where $\phi_{\mathrm{SMSN}}($.$) is the pdf of a SMSN distribution.$

The mean and variance are respectively given by $\mathrm{E}[T]=\frac{\beta}{2}\left[2+\alpha^{2} \mu_{2}+\alpha \omega_{1}\right]$ and $\operatorname{Var}[T]=$ $\frac{\beta^{2}}{4}\left[\left(4 \alpha^{2}-2 \alpha^{3} \omega_{1}\right) \mu_{2}-\alpha^{4} \mu_{2}^{2}+2 \alpha^{4} \mu_{4}-\alpha^{2} \omega_{1}^{2}+2 \alpha^{3} \omega_{3}\right]$, where $\mu_{j}=\mathrm{E}\left(Z^{j}\right), j=2,4$ and $\omega_{k}=$ $\mathbb{E}\left[Z^{k} \sqrt{\alpha^{2} Z^{2}+4}\right]$, for $k=1,3$, with $Z \sim \operatorname{SMSN}(0,1, \lambda ; G)$.

In the next section, we present some additional special cases of the SMSN distributions. We also compute the conditional moments defined by $\kappa_{r}=\mathrm{E}\left[\kappa^{r}(U) \mid t\right]$ and $\tau_{r}=\mathrm{E}\left[\kappa^{r / 2}(U) W_{\Phi}\left(\kappa^{1 / 2}(U) B_{t}\right) \mid t\right]$, 
where $B_{t}=\lambda a_{t}(\alpha, \beta)$ and $W_{\Phi}(x)=\phi(x) / \Phi(x)$, with $x \in \mathbb{R}$. Derivation of these moments will be useful in the implementation of the EM algorithm and the estimation of the latent variable $x$.

Theorem 2. Let $T \sim \operatorname{SMSN-BS}(\alpha, \beta, \lambda ; G)$ and $H \mid(U=u) \sim \operatorname{HN}(0, \kappa(u))$. Then, $T \mid(H=h, U=$ u) has a p.d.f. as

$$
\left.f_{T \mid H, U}(t \mid h, u)=\phi\left(\lambda_{h, u}+a_{t}\left(\alpha_{\delta, u}, \beta\right)\right) A_{t} \alpha_{\delta, u}, \beta\right), \quad t>0,
$$

where $\alpha_{\delta, u}=\alpha \kappa^{1 / 2}(u) \sqrt{1-\delta^{2}}, \lambda_{h, u}=-\delta h /\left(\kappa^{1 / 2}(u) \sqrt{1-\delta^{2}}\right), a_{t}\left(\alpha_{\delta, u}, \beta\right)$ and $A_{t}\left(\alpha_{\delta, u}, \beta\right)$ are as given in (2.1) with $\alpha_{\delta, u}$ instead of $\alpha$, and $\delta=\lambda / \sqrt{1+\lambda^{2}}$.

The conditional pdf, given in Theorem 2 , is of a positive random variable that has an extended Birnbaum-Saunders (EBS) distribution as proposed by Leiva et al. (2010), and the distribution in (2.8) is denoted by $\operatorname{EBS}\left(\alpha_{\delta, u}, \beta, \sigma=2, \lambda_{h, u}\right)$.

Theorem 3. Let $T \sim \operatorname{SMSN-BS}(\alpha, \beta, \lambda ; G)$. Then, $H \mid(T=t, U=u)$ has a standard HN distribution with pdf given by

$$
f_{H \mid T, U}(h \mid t, u)=\frac{\phi\left(h \mid \delta \kappa^{1 / 2}(u) a_{t}\left(\alpha_{u}, \beta\right), \kappa(u)\left(1-\delta^{2}\right)\right)}{\Phi\left(\lambda a_{t}\left(\alpha_{u}, \beta\right)\right)}, \quad t>0,
$$

where $\alpha_{u}=\alpha \sqrt{\kappa(u)}$ and $\phi\left(\cdot \mid \mu, \sigma^{2}\right)$ denotes the normal pdf with mean $\mu$ and variance $\sigma^{2}$. Hence,

$$
\mathrm{E}\left[\kappa^{-1}(U) H \mid T=t\right]=\delta a_{t}(\alpha, \beta) \kappa+\tau \sqrt{1-\delta^{2}}
$$

and

$$
\mathrm{E}\left[\kappa^{-1}(U) H^{2} \mid T=t\right]=\delta^{2} a_{t}^{2}(\alpha, \beta) \kappa+\left[1-\delta^{2}\right]+\delta a_{t}(\alpha, \beta) \tau \sqrt{1-\delta^{2}},
$$

where $\kappa=\mathrm{E}\left[\kappa^{-1}(U) \mid T=t\right], \tau=\mathrm{E}\left[\kappa^{-1 / 2}(U) W_{\Phi}\left(\frac{\delta a_{t}(\alpha, \beta)}{\kappa^{1 / 2}(u) \sqrt{1-\delta^{2}}}\right) \mid T=t\right]$ and $W_{\Phi}(u)=\phi(u) / \Phi(u)$, for $u \in \mathbb{R}$.

Proof. From the stochastic representation in (2.5), we have that the conditional distribution $T$, given $U=u$, is the BS distribution of Vilca et al. (2011). That is, $T \mid(U=u) \sim \operatorname{SN}-\operatorname{BS}\left(\alpha_{u}, \beta, \lambda\right)$, and its pdf is

$$
f_{T \mid U}(t \mid u)=2 \phi\left(a_{t}\left(\alpha_{u}, \beta\right)\right) \Phi\left(\lambda a_{t}\left(\alpha_{u}, \beta\right)\right) A_{t}\left(\alpha_{u}, \beta\right) .
$$


Consequently, the conditional pdf of $H$, given $T=t, U=u$, can be expressed as

$$
f_{H \mid T, U}(h \mid t, u)=\frac{f_{T \mid H, U}(t \mid h, u) f_{H \mid U}(h \mid u)}{f_{T \mid U}(t \mid u)},
$$

Then, the pdf in (2.9) is obtained by using the results presented in (2.10) and (2.11). Finally, the conditional expectations are derived from the following results $\mathrm{E}\left[\kappa^{-1}(U) H \mid t\right]=\mathrm{E}\left[\kappa^{-1}(U) \mathrm{E}(H \mid T, U) \mid t\right]$ and $\mathrm{E}\left[\kappa^{-1}(U) H^{2} \mid t\right]=\mathrm{E}\left[\kappa^{-1}(U) \mathrm{E}\left(H^{2} \mid T, U\right) \mid t\right]$. Specifically, we first use the conditional pdf of $H$, given $\mathrm{T}$ and $\mathrm{U}$, to obtain

$$
\mathrm{E}\left[\kappa^{-1}(U) H \mid t\right]=\delta a_{t}(\alpha, \beta) \mathrm{E}\left[\kappa^{-1}(U) \mid t\right]+\sqrt{1-\delta^{2}} \mathrm{E}\left[\kappa^{-1 / 2}(U) W_{\Phi}\left(\frac{\kappa^{-1 / 2}(U) \delta a_{t}(\alpha, \beta)}{\sqrt{1-\delta^{2}}}\right) \mid t\right]
$$

and

$$
\begin{aligned}
\mathrm{E}\left[\kappa^{-1}(U) H^{2} \mid t\right]= & \left(\delta a_{t}(\alpha, \beta)\right)^{2} \mathrm{E}\left[\kappa^{-1}(U) \mid t\right]+1-\delta^{2} \\
& +\delta a_{t}(\alpha, \beta) \sqrt{1-\delta^{2}} \mathrm{E}\left[\kappa^{-1 / 2}(U) W_{\Phi}\left(\frac{\kappa^{-1 / 2}(U) \delta a_{t}(\alpha, \beta)}{\sqrt{1-\delta^{2}}}\right) \mid t\right]
\end{aligned}
$$

Next, we present the mean and variance of the $T \sim \operatorname{SMSN}-B S(\alpha, \beta, \lambda ; G)$.

Theorem 4. Let $T \sim \operatorname{SMSN-BS}(\alpha, \beta, \lambda ; G)$. Then,

(i) if $\Phi_{\mathrm{SMSN}}(. ; \lambda)$ is the cdf of the $S M S N$ distribution, then the cdf of $T$ is given by $F_{T}(t ; \alpha, \beta, \lambda)=$ $\Phi_{\mathrm{SMSN}}\left(a_{t}(\alpha, \beta) ; \lambda\right)$. Moreover,

a) $F_{T}(t ; \alpha, \beta,-\lambda)=2 \Phi_{\mathrm{SMN}}\left(a_{t}(\alpha, \beta)\right)-\Phi_{\mathrm{SMSN}}\left(a_{t}(\alpha, \beta) ; \lambda\right)$, where $\Phi_{\mathrm{SMN}}($.$) is the cdf of the$ SMN distribution;

b) $F_{T}(t ; \alpha, \beta, \lambda=1)=\Phi_{\mathrm{SMN}}^{2}\left(a_{t}(\alpha, \beta)\right)$;

c) Let $S \sim \operatorname{SMSN-BS}(\alpha, \beta, \lambda ; G)$ independent of $T$. Then, $V=\max (T, S)$ has density given by

$$
f_{V}(v)=2 \phi_{\mathrm{SMSN}}\left(a_{t}(\alpha, \beta)\right) \Phi_{\mathrm{SMSN}}\left(a_{t}(\alpha, \beta)\right) A_{t}(\alpha, \beta)
$$

(ii) if there is $\boldsymbol{\nu}_{\infty}$ such as $\lim _{\boldsymbol{\nu} \rightarrow \boldsymbol{\nu}_{\infty}} \phi_{\mathrm{SMSN}}(z ; \lambda)=f_{0}(z ; \lambda)$ for all $z$, where $f_{0}(. ; \lambda)$ is the pdf of the $\operatorname{SN}(0,1, \lambda)$ distribution, then $\lim _{\boldsymbol{\nu} \rightarrow \boldsymbol{\nu}_{\infty}} f_{T}(t)=f_{S N}(t ; \lambda)$ for all $t>0$, where $f_{S N}(. ; \lambda)$ is the pdf of a $\mathrm{SN}-\mathrm{BS}(\alpha, \beta, \lambda)$ distribution; 
iii) the distribution of $d(T)=a_{T}^{2}(\alpha, \beta)=\frac{1}{\alpha^{2}}\left(\frac{T}{\beta}+\frac{\beta}{T}-2\right)$ does not depend on $\lambda$.

Remark 1. a) From Theorem 4 (i) and Vilca and Leiva (2006), the p-th percentile of the distribution of $T, t_{p}=F_{T}^{-1}(p ; \alpha, \beta, \lambda)$, is given by

$$
t_{p}=\frac{\beta}{4}\left(\alpha z_{p}+\sqrt{\alpha^{2} z_{p}^{2}+4}\right)^{2}
$$

where $z_{p}$ is the $p$-th percentile of the SMSN distribution. For $\lambda=0$, the distribution of $T$ reduces to that based on the $S M N$ distribution, and so $t_{0.5}=\beta$;

b) The distribution of $V$ in Theorem 4 (i)(Part c), is a type of Birnbaum-Saunders distribution based on a generalized skew-normal distribution (Gupta and Gupta, 2008);

c) The result in Theorem 4 (iii) is extremely useful for testing the goodness of fit and for detecting outliers. In addition, the distribution of $d(T)$ is the same as under the BS based on the SMN distribution of Balakrishnan et al. (2009). For more information see Lange and Sinsheimer (1993).

\subsection{Special cases of the SMSN-BS family}

In this section, some special cases of the SMSN-BS family are discussed, which are based on the skew-normal (SN), skew-contaminated normal (SCN), skew-Slash (SSL) and skew-Student- $t$ (ST) distributions and $\kappa(U)=1 / U$. These cases are obtained from the stochastic representation given in (2.5) and Theorem 1. From Theorem 4 (iii) and Lange and Sinsheimer (1993), we have the distribution of $d(T)=a_{T}(\alpha, \beta)$, which is useful to obtain simulated envelopes, and it will be specified for each special case;

i) The skew-contaminated normal BS distribution

The SCN distribution can be utilized to generate a BS type distribution, which we call contaminated skew-normal Birnbaum-Saunders (SCN-BS) distribution. Here $U$ is a discrete random variable with pdf having the form

$$
h(u ; \nu, \gamma)=\nu \mathbb{I}_{\{\gamma\}}(u)+(1-\nu) \mathbb{I}_{\{1\}}(u), \quad 0<\nu<1,0<\gamma<1,
$$


where $\mathbb{I}_{A}(\cdot)$ denotes the indicator function of the set $A$. Then, the pdf of $T$ is obtained from (2.7) and can be expressed as

$$
f_{T}(t)=2\left[\nu \phi\left(a_{t}(\alpha, \beta) ; 0, \frac{1}{\gamma}\right) \Phi\left(\gamma^{1 / 2} B_{t}\right)+(1-\nu) \phi\left(a_{t}(\alpha, \beta)\right) \Phi\left(B_{t}\right)\right] A_{t}(\alpha, \beta) .
$$

For this distribution we use the notation $T \sim \mathrm{SNC}-\mathrm{BS}(\alpha, \beta, \lambda ; \nu, \gamma)$. In this case, we have

$$
\kappa_{r}=\frac{2 A_{t}(\alpha, \beta)}{f_{T}(t)}\left[\nu \gamma^{r} \phi\left(a_{t}(\alpha, \beta) ; 0, \gamma^{-1}\right) \Phi\left(\gamma^{1 / 2} B_{t}\right)+(1-\nu) \phi\left(a_{t}(\alpha, \beta)\right) \Phi\left(B_{t}\right)\right]
$$

and

$$
\tau_{r}=\frac{2 A_{t}(\alpha, \beta)}{f_{T}(t)}\left[\nu \gamma^{r / 2} \phi\left(a_{t}(\alpha, \beta) ; 0, \gamma^{-1}\right) \Phi\left(\gamma^{1 / 2} B_{t}\right)+(1-\nu) \phi\left(a_{t}(\alpha, \beta)\right) \Phi\left(B_{t}\right)\right] .
$$

The parameter $\nu$ can be interpreted as the proportion of outliers and $\gamma$ can be interpreted as a scale factor. When $\gamma=1$, the SCN-BS distribution reduces to the SN-BS distribution of Vilca et al. (2011). Finally, $d(T)$ has cdf given by $\operatorname{Pr}(d(T) \leq v)=\nu \operatorname{Pr}\left(\chi_{1}^{2} \leq \gamma v\right)+(1-\nu) \operatorname{Pr}\left(\chi_{1}^{2} \leq v\right)$

ii) The skew slash Birnbaum-Saunders distribution

The SSL distribution is used to generate a BS type distribution as follows. Consider $U$ having a $\operatorname{Beta}(\nu, 1)$ distribution, and the resulting distribution is called the skew-slash BirnbaumSaunders (SSL-BS) distribution. The pdf of $T$ has the form

$$
f_{T}(t)=2 \nu \int_{0}^{1} u^{\nu-1} \phi\left(a_{t}(\alpha, \beta) ; 0,1 / u\right) \Phi\left(u^{1 / 2} B_{t}\right) d u A_{t}(\alpha, \beta) .
$$

For this distribution, we use the notation $T \sim \mathrm{SSL}-\mathrm{BS}(\alpha, \beta, \lambda ; \nu)$. In this case, the conditional moments are given by

$$
\kappa_{r}=\frac{\nu \Gamma\left(\frac{2 \nu+2 r+1}{2}\right) A_{t}(\alpha, \beta)}{f_{T}(t) 2^{-(\nu+r+1)} \sqrt{\pi}} P_{1}\left(\frac{2 \nu+2 r+1}{2}, \frac{a_{t}^{2}(\alpha, \beta)}{2}\right) a_{t}(\alpha, \beta)^{-(2 \nu+2 r+1)} \mathrm{E}\left[\Phi\left(S^{1 / 2} B_{t}\right)\right]
$$

and

$$
\tau_{r}=\frac{\nu \Gamma\left(\frac{2 \nu+r+1}{2}\right) A_{t}(\alpha, \beta)}{f_{T}(t) 2^{-(\nu+r / 2+1 / 2)} \sqrt{\pi}^{2}}\left(a_{t}^{2}(\alpha, \beta)+B_{t}^{2}\right)^{-\frac{2 \nu+r+1}{2}} P_{1}\left(\frac{2 \nu+r+1}{2}, \frac{a_{t}^{2}(\alpha, \beta)+B_{t}^{2}}{2}\right)
$$

where $P_{x}(a, b)$ denotes the distribution function of the $\operatorname{gamma}(a, b)$ distribution evaluated at $x$ and $S \sim \operatorname{Gamma}\left(\frac{2 \nu+2 r+1}{2}, \frac{a_{t}^{2}(\alpha, \beta)}{2}\right) \mathbb{I}_{(0,1)}$, a truncated gamma distribution on $(0,1)$, with the parameter values in parentheses before truncation. In this case the cdf of $d(T)$ is 


$$
\operatorname{Pr}(d(T) \leq v)=\operatorname{Pr}\left(\chi_{1}^{2} \leq v\right)-\frac{2^{\nu} \Gamma(\nu+1 / 2)}{v^{\nu} \Gamma(1 / 2)} \operatorname{Pr}\left(\chi_{2 \nu+1}^{2} \leq v\right)
$$

iii) The skew-Student-t $B S$ distribution

The skew-Student- $t$ (ST) distribution has been used as an alternative model to the skewnormal one to obtain qualitatively robust parameter estimates. Special cases of the ST distribution are the skew-Cauchy model, when $\nu=1$, and the skew-normal model, when $\nu \rightarrow \infty$. The resulting BS type is called the skew-Student- $t$ Birnbaum-Saunders (ST-BS) distribution. In this case $U \sim \operatorname{Gamma}(\nu / 2, \nu / 2)$, and the pdf of $T$ is given by

$$
f_{T}(t)=2 t\left(a_{t}(\alpha, \beta) \mid 0,1 ; \nu\right) T\left(\sqrt{\frac{\nu+1}{a_{t}^{2}(\alpha, \beta)+\nu}} B_{t} ; \nu+1\right) A_{t}(\alpha, \beta), t>0,
$$

where $t(. \mid 0,1 ; \nu)$ and $T(. ; \nu)$ denote, respectively, the pdf and cdf of the standard Student- $t$ distribution. In this case, the conditional expectations are given by

$$
\kappa_{r}=\frac{\Gamma\left(\frac{\nu+2 r+1}{2}\right)\left(a_{t}^{2}(\alpha, \beta)+\nu\right)^{-\frac{\nu+2 r+1}{2}} A_{t}(\alpha, \beta)}{f_{T}(t) 2^{-(r+1)} \nu^{-\nu / 2} \Gamma(\nu / 2) \sqrt{(\pi)}} T\left(\sqrt{\frac{\nu+2 r+1}{a_{t}^{2}(\alpha, \beta)+\nu}} B_{t} ; \nu+2 r+1\right)
$$

and

$$
\tau_{r}=\frac{2^{(r+1) / 2} \nu^{\nu / 2} \Gamma\left(\frac{\nu+r+1}{2}\right)\left(a_{t}^{2}(\alpha, \beta)+\nu+B_{t}^{2}\right)^{-\frac{\nu+r+1}{2}} A_{t}(\alpha, \beta)}{f_{T}(t) \Gamma(\nu / 2) \sqrt{(\pi)}^{2}} .
$$

Finally, in this distribution $d(T) \sim F(1, \nu)$.

Figures 2.1 and 2.2 show the densities plots, for some values of the parameters, of the distributions ST-BS, SCN-BS and SSL-BS. They are rescaled so they have the same value at the origin. From Figure 2.2 (a) we can conclude that if the distribution of data is concentrated close of zero, then the SN-BS distribution, with negative $\lambda=-3$ may be more appropriate to fit the data than the BS or t-BS distribution, while on the other hand for positive $\lambda$ the SN-BS density function tends to become more leptokurtic than the BS or St-BS density functions, as can be seen in Figure 2.1 (a). Moreover, from Part (b)-(d) of the figures, we can see that the SMSN-BS distribution possesses heavier tails than the SN-BS distribution. 


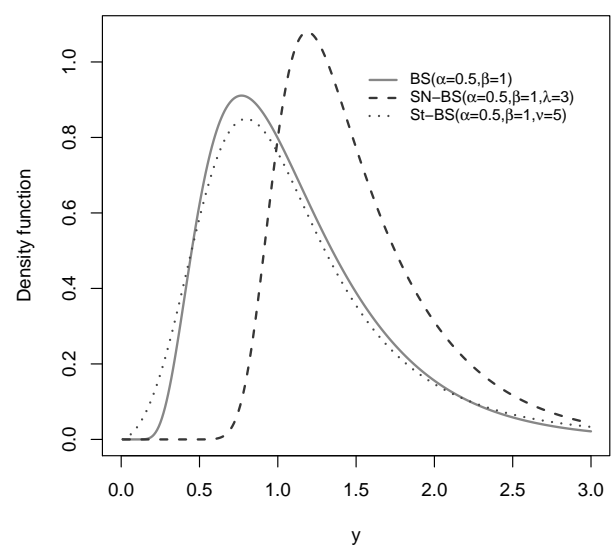

(a)

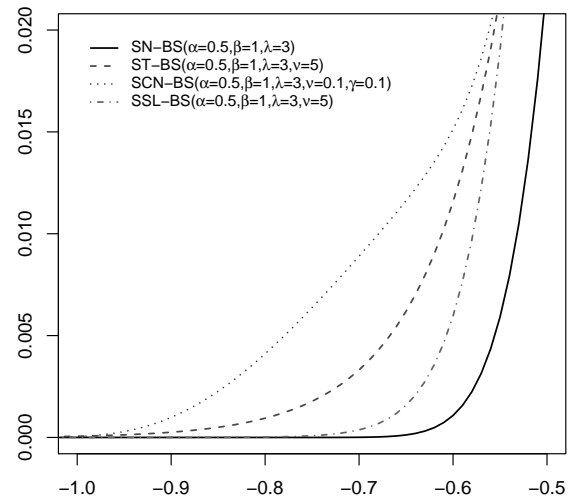

(c)

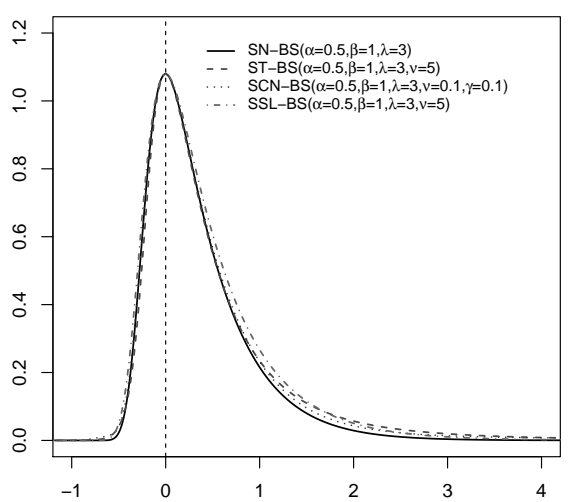

(b)

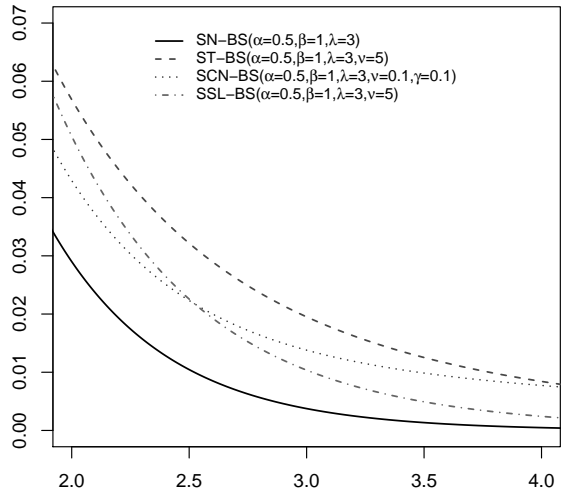

(d)

Figure 2.1: Density plots of: (a) BS, SN-BS and St-BS distributions, (b) $S N-B S, S T-B S, S N C-B S$ and SSL-BS distributions. (c) and (d) show left and right tails of the densities, all for $\lambda=3$. 


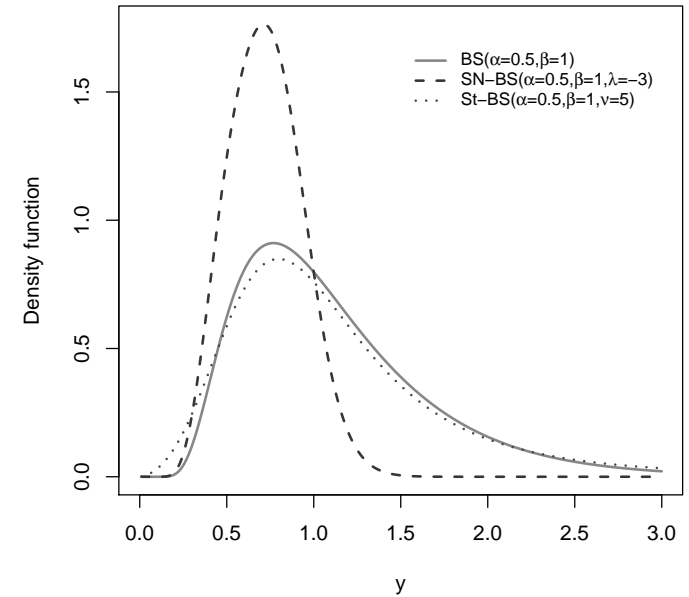

(a)

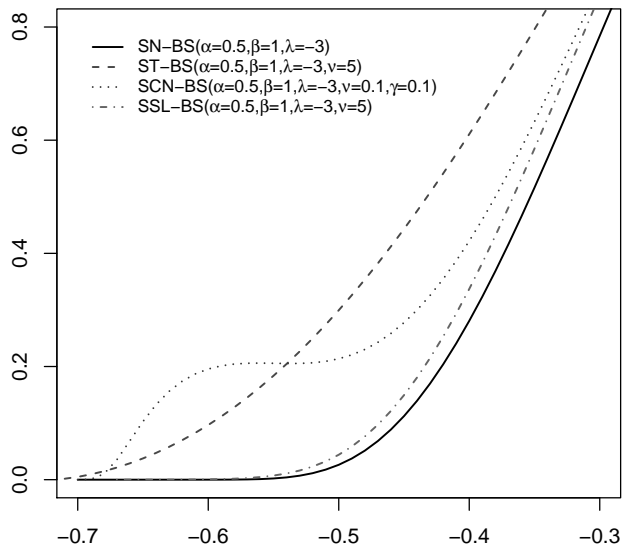

(c)

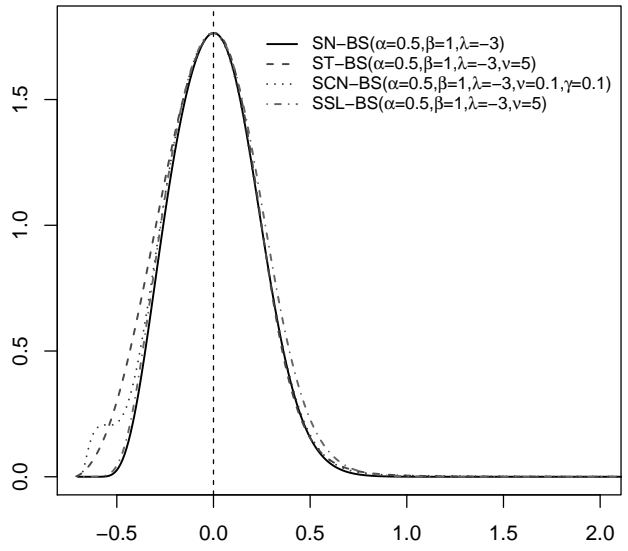

(b)

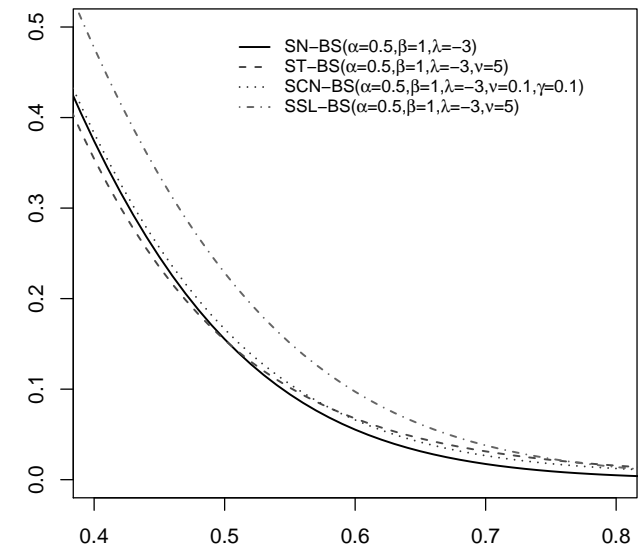

(d)

Figure 2.2: Density plots of: (a) $B S, S N-B S$ and $S t-B S$ distributions, (b) $S N-B S, S T-B S, S N C-B S$ and $S S L-B S$ distributions. (c) and (d) show left and right tails of the densities, all for $\lambda=-3$.

\subsection{Maximum likelihood estimation}

In this section, we discuss the ML estimation for the parameters of the SMSN-BS model through an EM algorithm. Let $T_{1}, \ldots, T_{n}$ be a random sample of size $n$ such as $T_{i} \sim \operatorname{SMSN-BS}(\alpha, \beta, \lambda ; G)$, for $i=1, \ldots, n$. Thus, the log-likelihood function for $\boldsymbol{\theta}=(\alpha, \beta, \lambda)^{\top}$ is $\ell(\boldsymbol{\theta})=\sum_{i=1}^{n} \ell_{i}(\boldsymbol{\theta})$, where $\ell_{i}(\boldsymbol{\theta})=\log \left(\phi_{S M S N}\left(a_{t_{i}}(\alpha, \beta)\right)\right)+\log \left(A_{t_{i}}(\alpha, \beta)\right), i=1, \ldots, n$. 


\subsubsection{The ML estimation based on the EM-algorithm}

The EM-algorithm is a well-known technique for ML estimation when unobserved (or missing) data or latent variables are present while modeling. This algorithm enables computationally efficient determination of the ML estimates when iterative methods are required. Specifically, let $\mathbf{t}_{o}=\left(t_{1}, \ldots, t_{n}\right)^{\top}$, and $\mathbf{h}=\left(h_{1}, \ldots, h_{n}\right)^{\top}$ and $\mathbf{u}=\left(u_{1}, \ldots, u_{n}\right)^{\top}$ denote observed and unobserved data, respectively. The complete data $\mathbf{t}_{c}=\left(\mathbf{t}_{o}, \mathbf{h}, \mathbf{u}\right)$ corresponds to the original observed data $\mathbf{t}_{o}$ augmented with $\mathbf{h}$ and $\mathbf{u}$. We now describe the implementation of the EM-algorithm for the ML estimation of the parameters of SMSN-BS distributions, following the ideas of Vilca et al. (2011). Note that the SMSN-BS model can be written hierarchically as

$$
\begin{aligned}
T_{i} \mid\left(H_{i}=h_{i}, U_{i}=u_{i}\right) & \stackrel{\text { ind }}{\sim} \operatorname{EBS}\left(\alpha_{\delta, u_{i}}, \beta, \sigma=2, \lambda_{h_{u_{i}}}\right), \\
H_{i} \mid U_{i}=u_{i} & \stackrel{\text { iid }}{\sim} \operatorname{HN}\left(0, \kappa\left(u_{i}\right)\right), \\
U_{i} & \sim H\left(u_{i}, \boldsymbol{\nu}\right) \quad i=1, \ldots, n,
\end{aligned}
$$

where $\alpha_{\delta, u_{i}}=\alpha \kappa^{1 / 2}\left(u_{i}\right) \sqrt{1-\delta^{2}}$ and $\lambda_{h_{u_{i}}}=-\delta h_{i} /\left(\kappa^{1 / 2}\left(u_{i}\right) \sqrt{1-\delta^{2}}\right)$. Then, under the hierarchical representation given in (2.14)-(2.16), it follows that the complete-data log-likelihood function associated with $\mathbf{t}_{c}$ can be expressed as

$$
\ell_{c}\left(\boldsymbol{\theta} \mid \mathbf{t}_{c}\right)=c-n \log \left(\alpha_{\delta}\right)+\sum_{i=1}^{n} \log \left(\frac{t_{i}+\beta}{\sqrt{\beta}}\right)-\frac{1}{2\left(1-\delta^{2}\right)} \sum_{i=1}^{n} \frac{1}{\kappa\left(u_{i}\right)}\left(a_{t_{i}}(\alpha, \beta)-\delta h_{u_{i}}\right)^{2}
$$

where $c$ is a constant that does not depend on $\boldsymbol{\theta}$. Let $\widehat{\boldsymbol{\theta}}^{(k)}=\left(\widehat{\alpha}^{(k)}, \widehat{\beta}^{(k)}, \widehat{\delta}^{(k)}\right)^{\top}$ be the ML estimate of $\boldsymbol{\theta}=(\alpha, \beta, \delta)^{\top}$ at the $k$-th iteration.

The conditional expectation of the complete log-likelihood function given by $Q(\boldsymbol{\theta} \mid \widehat{\boldsymbol{\theta}})=\mathbb{E}\left[\ell_{c}\left(\boldsymbol{\theta} \mid \mathbf{t}_{c}\right) \mid \widehat{\boldsymbol{\theta}}\right]=$ $\sum_{i=1}^{n} Q_{i}(\boldsymbol{\theta} \mid \widehat{\boldsymbol{\theta}})$, where

$$
Q_{i}(\boldsymbol{\theta} \mid \widehat{\boldsymbol{\theta}})=c-\log \left(\alpha_{\delta}\right)+\log \left(\frac{t_{i}+\beta}{\sqrt{\beta}}\right)-\frac{1}{2 \alpha_{\delta}^{2}} \widehat{\kappa}_{i} \xi^{2}\left(t_{i} ; \beta\right)+\frac{\lambda}{\alpha_{\delta}} \widehat{h}_{u_{i}} \xi\left(t_{i} ; \beta\right)-\frac{\lambda^{2}}{2} \widehat{h}^{2} u_{i},
$$

with $\xi\left(t_{i} ; \beta\right)=\sqrt{t_{i} / \beta}-\sqrt{\beta / t_{i}}$ and $\lambda=\delta / \sqrt{1-\delta^{2}}$. Hence, calculation of $Q(\boldsymbol{\theta} \mid \widehat{\boldsymbol{\theta}})$ requires the expressions $\widehat{\kappa}_{i}=\mathrm{E}\left[\kappa^{-1}\left(U_{i}\right) \mid \widehat{\boldsymbol{\theta}}^{(k)}, t_{i}\right], \widehat{h}_{u_{i}}=\mathrm{E}\left[\kappa^{-1}\left(U_{i}\right) H_{i} \mid \widehat{\boldsymbol{\theta}}^{(k)}, t_{i}\right]$ and $\widehat{h_{u_{i}}^{2}}=\mathrm{E}\left[\kappa^{-1}\left(U_{i}\right) H_{i}^{2} \mid \widehat{\boldsymbol{\theta}}^{(k)}, t_{i}\right]$, $i=1, \ldots, n$. By using properties of the conditional distributions and using results in Theorem 3, 
the two last terms are readily evaluated to be

$$
\begin{aligned}
\widehat{h}_{u_{i}} & =\widehat{\delta} a_{t_{i}}(\widehat{\alpha}, \widehat{\beta}) \widehat{\kappa}_{i}+\widehat{\tau}_{i} \sqrt{1-\widehat{\delta}^{2}} \\
{\widehat{h^{2}}}_{u_{i}} & =\widehat{\delta}^{2} a_{t_{i}}^{2}(\widehat{\alpha}, \widehat{\beta}) \widehat{\kappa}_{i}+\left[1-\widehat{\delta}^{2}\right]+\widehat{\delta} a_{t_{i}}(\widehat{\alpha}, \widehat{\beta}) \widehat{\tau}_{i} \sqrt{1-\widehat{\delta}^{2}}
\end{aligned}
$$

We can see that the quantities $\widehat{\kappa}_{i}, \widehat{h}_{u_{i}}$ and ${\widehat{h^{2}}}_{u_{i}}$ depend on the distribution of $U$. Thus, we then have the following EM algorithm:

E-step. Given $\boldsymbol{\theta}=\widehat{\boldsymbol{\theta}}^{(k)}$, compute $\widehat{\kappa}_{i}^{(k)}, \widehat{h}_{i}^{(k)}$ and ${\widehat{h^{2}}}^{(k)}$, for $i=1, \ldots, n$, using results in (2.17) and $(2.18)$;

CM-step 1. Fix $\widehat{\beta}^{(k)}$ and update $\widehat{\alpha}^{(k)}$ and $\widehat{\delta}^{(k)}$ as

$$
\begin{aligned}
{\widehat{\alpha^{2}}}^{(k+1)} & =\frac{1}{n} \sum_{i=1}^{n} \widehat{\kappa}_{i} \xi^{2}\left(t_{i} ; \widehat{\beta}^{(k)}\right)+\left[1-\widehat{\tau}_{h}\right]\left[\frac{\sum_{i=1}^{n}{\widehat{h_{u_{i}}}}^{(k)} \xi\left(t_{i} ; \widehat{\beta}^{(k)}\right)}{n \widehat{\tau}_{h_{u_{i}}}}\right]^{2} \text { and } \\
\widehat{\delta}^{(k+1)} & =\frac{1}{\widehat{\alpha}^{(k+1)}} \frac{\sum_{i=1}^{n} \widehat{h}_{u_{i}}^{(k)} \xi\left(t_{i} ; \widehat{\beta}^{(k)}\right)}{n \widehat{\tau}_{h}}
\end{aligned}
$$

where $\widehat{\tau}_{h}=\frac{\sum_{i=1}^{n}{\widehat{h^{2}}}_{u_{i}}^{(k)}}{n}$;

CM-step 2. Fix $\widehat{\alpha}^{(k+1)}$ and $\widehat{\delta}^{(k+1)}$ and update $\widehat{\beta}^{(k)}$ using

$$
\widehat{\beta}^{(k+1)}=\underset{\beta}{\operatorname{argmax}} Q\left(\widehat{\alpha}^{(k+1)}, \beta, \widehat{\delta}^{(k+1)} \mid \widehat{\boldsymbol{\theta}}^{(k)}\right) .
$$

The iterations are repeated until a suitable convergence is attained based on the requirement $\left\|\widehat{\boldsymbol{\theta}}^{(k+1)}-\widehat{\boldsymbol{\theta}}^{(k)}\right\|<10^{-8}$

Remark 2. From the above EM algorithm, it is important to point out some points for this iterative optimization procedures that we present next:

(i) In the EM algorithm, we get the $M L$ estimate for the parameter $\delta$. Thus, because of the invariance property of the $M L$ estimates and by using (2.2), we have that the ML estimate of $\lambda$ given by

$$
\widehat{\lambda}=\widehat{\delta} / \sqrt{1-\widehat{\delta}^{2}}
$$

(ii) The estimate $\boldsymbol{\nu}$ is obtained through the profiled maximized log-likelihood in function of $\boldsymbol{\nu}$, as in Balakrishnan et al. (2009). For example, for the ST-BS and SSL-BS models, we have fixed integer values for $\nu$ from 3 to 100, and 2 to 100, respectively, choosing the value of $\nu$ that maximizes the likelihood function; 
(iii) Useful starting values are required to implement the above EM-algorithm, which may be obtained following the idea in Vilca et al. (2011), which is implemented in the function mmmeth () in the $\mathbf{R}$ package bssn (Maehara and Benites, 2015). First, we utilize the modified moment estimates proposed by $\mathrm{Ng}$ et al. (2003) to obtain the initial values for $\alpha$ and $\beta$, and then is used the $\mathbf{R}$ package $\mathbf{b s s n}$ (Maehara and Benites, 2015) to obtain an estimate for $\lambda$ under the SN-BS model. All these values are the initial values of the parameters. In general, if $\widehat{\alpha}^{(0)}$ and $\widehat{\beta}^{(0)}$ are the modified moment estimates of $\alpha$ and $\beta$, respectively, then by considering the random variables

$$
Z_{i}=\frac{1}{\widehat{\alpha}^{(0)}}\left(\sqrt{T_{i} / \widehat{\beta}^{(0)}}-\sqrt{\widehat{\beta}^{(0)} / T_{i}}\right) \sim \operatorname{SMSN}(0,1, \lambda ; G), i=1, \ldots, n,
$$

we obtain the estimates of $\lambda$ through EM algorithm in Basso et al. (2009), that is denoted by $\widehat{\lambda}^{(0)}$.

\subsubsection{The observed information matrix}

In this section we obtain the observed information matrix of the SMSN-BS model, defined as $\mathbf{J}_{o}(\boldsymbol{\theta} \mid \mathbf{t})=-\partial^{2} \ell(\boldsymbol{\theta} \mid \mathbf{t}) / \partial \boldsymbol{\theta} \partial \boldsymbol{\theta}^{\top}$. It is well known that, under some regularity conditions, the covariance matrix of the maximum likelihood estimates $\widehat{\boldsymbol{\theta}}$ can be approximated by the inverse of $\mathbf{J}_{o}(\boldsymbol{\theta} \mid \mathbf{t})$. Following Basford et al. (1997) and Lin et al. (2007), we evaluate

$$
\mathbf{J}_{o}(\widehat{\boldsymbol{\theta}} \mid \mathbf{t})=\sum_{i=1}^{n} \widehat{\mathbf{s}}_{i} \widehat{\mathbf{s}}_{i}^{\top},
$$

where $\widehat{\mathbf{s}}_{i}=\partial \log f\left(t_{i} ; \boldsymbol{\theta}\right) /\left.\partial \boldsymbol{\theta}\right|_{\boldsymbol{\theta}=\widehat{\boldsymbol{\theta}}}$. We consider now the vector $\widehat{\mathbf{s}}_{i}$ partitioned in term of components of the parameters in $\boldsymbol{\theta}$ as $\widehat{\mathbf{s}}_{i}=\left(\widehat{s}_{i, \alpha}, \widehat{s}_{i, \beta}, \widehat{s}_{i, \lambda}\right)^{\top}$, whose components are given by

$$
\widehat{s}_{i, \alpha}=\frac{D_{\alpha} f\left(t_{i} ; \boldsymbol{\theta}\right)}{f\left(t_{i} ; \boldsymbol{\theta}\right)}, \quad \widehat{s}_{i, \beta}=\frac{D_{\beta} f\left(t_{i} ; \boldsymbol{\theta}\right)}{f\left(t_{i} ; \boldsymbol{\theta}\right)} \text { and } \widehat{s}_{i, \lambda}=\frac{D_{\lambda} f\left(t_{i} ; \boldsymbol{\theta}\right)}{f\left(t_{i} ; \boldsymbol{\theta}\right)}
$$

where $D_{\alpha} f\left(t_{i} ; \boldsymbol{\theta}\right)=\frac{\partial}{\partial \alpha} f\left(t_{i} ; \boldsymbol{\theta}\right), D_{\beta} f\left(t_{i} ; \boldsymbol{\theta}\right)=\frac{\partial}{\partial \beta} f\left(t_{i} ; \boldsymbol{\theta}\right)$ and $D_{\lambda} f\left(t_{i} ; \boldsymbol{\theta}\right)=\frac{\partial}{\partial \lambda} f\left(t_{i} ; \boldsymbol{\theta}\right)$. Let us define

$$
I_{i}^{\Phi}(w)=\int_{0}^{\infty} \kappa^{-w}(u) \exp \left\{-\frac{1}{2} \kappa^{-1}(u) a_{t_{i}}^{2}(\alpha, \beta)\right\} \Phi\left(\kappa^{-1 / 2}(u) B_{t_{i}}\right) d H(u)
$$

and

$$
I_{i}^{\phi}(w)=\int_{0}^{\infty} \kappa^{-w}(u) \exp \left\{-\frac{1}{2} \kappa^{-1}(u) a_{t_{i}}^{2}(\alpha, \beta)\right\} \phi\left(\kappa^{-1 / 2}(u) B_{t_{i}}\right) d H(u),
$$


where $B_{t_{i}}=\lambda a_{t_{i}}(\alpha, \beta), \quad i=1, \ldots, n$. After some algebraic manipulation, we obtain

$$
\begin{aligned}
D_{\alpha}\left(f\left(t_{i} ; \boldsymbol{\theta}\right)\right) & =\frac{2}{\sqrt{2 \pi}}\left[\frac{\partial A_{t_{i}}}{\partial \alpha} I_{i}^{\Phi}(1 / 2)-a_{t_{i}} A_{t_{i}} \frac{\partial a_{t_{i}}}{\partial \alpha} I_{i}^{\Phi}(3 / 2)+\lambda A_{t_{i}} \frac{\partial a_{t_{i}}}{\partial \alpha} I_{i}^{\phi}(1)\right], \\
D_{\beta}\left(f\left(t_{i} ; \boldsymbol{\theta}\right)\right) & =\frac{2}{\sqrt{2 \pi}}\left[\frac{\partial A_{t_{i}}}{\partial \beta} I_{i}^{\Phi}(1 / 2)-a_{t_{i}} A_{t_{i}} \frac{\partial a_{t_{i}}}{\partial \beta} I_{i}^{\Phi}(3 / 2)+\lambda A_{t_{i}} \frac{\partial a_{t_{i}}}{\partial \beta} I_{i}^{\phi}(1)\right], \\
D_{\lambda}\left(f\left(t_{i} ; \boldsymbol{\theta}\right)\right) & =\frac{2}{\sqrt{2 \pi}} a_{t_{i}} A_{t_{i}} I_{i}^{\phi}(1) .
\end{aligned}
$$

Following Lachos et al. (2010a), we obtain a closed-form expression for the quantities $I_{i}^{\Phi}(w)$ and $I_{i}^{\phi}(w)$, for the SNC-BS, SSL-BS and ST-BS distributions:

(i) The $S C N-B S$ distribution

$$
\begin{aligned}
& I_{i}^{\Phi}(w)=\sqrt{2 \pi}\left\{\nu \gamma^{w-1 / 2} \phi\left(a_{t_{i}}(\alpha, \beta) ; 0, \frac{1}{\gamma}\right) \Phi\left(\gamma^{1 / 2} B_{t_{i}}\right)+(1-\nu) \phi\left(a_{t_{i}}(\alpha, \beta)\right) \Phi\left(B_{t_{i}}\right)\right\}, \\
& I_{i}^{\phi}(w)=\nu \gamma^{w-1 / 2} \phi\left(\left(a_{t_{i}}^{2}(\alpha, \beta)+B_{t_{i}}^{2}\right)^{1 / 2} ; 0, \frac{1}{\gamma}\right)+(1-\nu) \phi\left(\left(a_{t_{i}}^{2}(\alpha, \beta)+B_{t_{i}}^{2}\right)^{1 / 2} ; 0,1\right) .
\end{aligned}
$$

(ii) The $S S L-B S$ distribution

$$
\begin{aligned}
I_{i}^{\Phi}(w) & =\frac{2^{2+\nu} \Gamma(w+\nu)}{\left[a_{t_{i}}(\alpha, \beta)\right]^{2 w+2 \nu}} P_{1}\left(w+\nu, a_{t_{i}}^{2}(\alpha, \beta) / 2\right) E\left[\Phi\left(S_{i}^{1 / 2}\right) B_{t_{i}}\right] \\
I_{i}^{\phi}(w) & =\frac{\nu 2^{w+\nu} \Gamma(w+\nu)}{\sqrt{2 \pi}\left(a_{t_{i}}^{2}(\alpha, \beta)+B_{t_{i}}^{2}\right) w+\nu} P_{1}\left(w+\nu, \frac{a_{t_{i}}^{2}(\alpha, \beta)+B_{t_{i}}^{2}}{2}\right) .
\end{aligned}
$$

(iii) The $S T$-BS distribution

$$
\begin{aligned}
I_{i}^{\Phi}(w) & =\frac{2^{w} \nu^{\nu / 2} \Gamma(w+\nu / 2)}{\Gamma(\nu / 2)\left(\nu+a_{t_{i}}^{2}(\alpha, \beta)\right)^{\nu / 2+w}} T\left(\frac{B_{t_{i}}}{\left(a_{t_{i}}^{2}(\alpha, \beta)+\nu\right)^{1 / 2}} \sqrt{2 w+\nu} ; 2 w+\nu\right), \\
I_{i}^{\phi}(w) & =\frac{2^{w} \nu^{\nu / 2}}{\sqrt{2 \pi} \Gamma(\nu / 2)}\left(\frac{1}{a_{t_{i}}^{2}(\alpha, \beta)+B_{t_{i}}^{2}+\nu}\right)^{\frac{\nu+2 w}{2}} \Gamma\left(\frac{\nu+2 w}{2}\right) .
\end{aligned}
$$

\subsection{Simulation study}

In this section, we carry out a numerical illustration to evaluate the performance of the method developed in the preceding sections, showing the quality of the estimation method through the EM algorithm proposed in Section 2.4 and the finite-sample performance of the estimates by means of Monte Carlo simulations. 


\subsubsection{Experiment 1: Parameter recovery}

We simulated samples of size $n=500$ from the SMSN-BS distribution using $M=1000$ replications. The true parameter values are $\alpha=0.5, \beta=1$ and $\lambda=1.5$ under the ST-BS $(\nu=3$ and $\nu=5)$, SSL-BS $(\nu=3$ and $\nu=5)$ and SCN-BS $(\nu=0.1$ and $\gamma=0.1$; and $\nu=0.3$ and $\gamma=0.3$ ) models. For all scenarios, we computed the means of the parameter estimates, the square root of the mean square error (RMSE), the bias (Bias) and the Monte Carlo standard deviation (MC-Sd), for each parameter over the 1000 replicates. Moreover, via the observed Information Matrix (IM) derived in (2.19), we also computed the average value of the approximate standard error (IM-SE), and the percentage of times that the confidence intervals (CI) cover the true value of the parameter (COV-MC). All these quantities are presented in Table 2.1. Note that regardless of the fixed value of $\nu$, the results suggest that the proposed SMSN-BS model produced satisfactory estimates. We can also see in Table 2.1 that the estimation method of the standard errors provides relatively close results (IM SE and MC Sd) and the 95\% coverage probability (COV-MC) defined as $\operatorname{CP}(\hat{\theta})=\frac{1}{m} \sum_{j=1}^{m} I\left(\theta \in\left[\hat{\theta}_{L}, \hat{\theta}_{U}\right]\right)$, where $I$ is the indicator function such that $\theta$ lies in the interval $\left[\hat{\theta}_{L}, \hat{\theta}_{U}\right]$, with $\hat{\theta}_{L}$ and $\hat{\theta}_{U}$ the estimated lower and upper bounds of the $95 \%$ CI, respectively. The COV-MC for the parameters is quite stable, indicating that the proposed asymptotic approximations for the variance estimates of the ML estimates are reliable.

Table 2.1: Means and MC-Sd of EM estimates based on 1000 samples, with IM-SE (the average value of the approximate standard error obtained through the information-based method) and Cov-MC (the coverage probability) from fitting the model. True values of parameters are in parentheses.

\begin{tabular}{|c|c|c|c|c|c|c|c|c|c|}
\hline & & BS & SN-BS & \multicolumn{2}{|c|}{ ST-BS } & \multicolumn{2}{|c|}{ SCN-BS } & \multicolumn{2}{|c|}{ SSL-BS } \\
\hline & & & & \multicolumn{2}{|c|}{$\nu$} & \multicolumn{2}{|c|}{$(\nu, \gamma)$} & \multicolumn{2}{|c|}{$\nu$} \\
\hline Parameter & & & & 3 & 5 & $(0.1,0.1)$ & $(0.3,0.3)$ & 3 & 5 \\
\hline \multirow[t]{4}{*}{$\alpha(0.5)$} & Mean & 0.4989 & 0.5025 & 0.4980 & 0.4870 & 0.5025 & 0.5055 & 0.4996 & 0.4805 \\
\hline & IM-SE & 0.0160 & 0.0401 & 0.0386 & 0.0367 & 0.0406 & 0.0396 & 0.0448 & 0.0390 \\
\hline & MC-Sd & 0.0160 & 0.0341 & 0.0451 & 0.0387 & 0.0307 & 0.0283 & 0.0623 & 0.0512 \\
\hline & COV-MC & $94.9 \%$ & $96.8 \%$ & $90.6 \%$ & $89.4 \%$ & $98.90 \%$ & $99.50 \%$ & $82.3 \%$ & $82.9 \%$ \\
\hline \multirow[t]{4}{*}{$\beta(1.0)$} & Mean & 0.9994 & 0.9989 & 1.0060 & 1.0052 & 0.9980 & 0.9919 & 1.0127 & 1.0168 \\
\hline & IM-SE & 0.0217 & 0.0526 & 0.0468 & 0.0463 & 0.0501 & 0.0538 & 0.0717 & 0.0632 \\
\hline & MC-Sd & 0.0211 & 0.0433 & 0.0468 & 0.0360 & 0.0360 & 0.0351 & 0.0895 & 0.0723 \\
\hline & COV-MC & $94.9 \%$ & $94.8 \%$ & $94.8 \%$ & $97.3 \%$ & $99.47 \%$ & $99.33 \%$ & $94.1 \%$ & $95.5 \%$ \\
\hline \multirow[t]{4}{*}{$\lambda(1.5)$} & Mean & - & 1.5085 & 1.5037 & 1.5190 & 1.5476 & 1.5775 & 1.5116 & 1.4752 \\
\hline & IM-SE & - & 0.3914 & 0.3222 & 0.3284 & 0.3614 & 0.3612 & 0.4181 & 0.3806 \\
\hline & MC-Sd & - & 0.3339 & 0.3077 & 0.2509 & 0.2302 & 0.2061 & 0.4435 & 0.4061 \\
\hline & COV-MC & - & $97.4 \%$ & $95.3 \%$ & $97.9 \%$ & $99.86 \%$ & $99.72 \%$ & $97.5 \%$ & $97.8 \%$ \\
\hline
\end{tabular}




\subsubsection{Experiment 2: Asymptotic properties}

The main focus in this simulation study is to show the asymptotic properties of the ML estimates. Our strategy is to consider artificial samples of SMSN-BS models, by choosing samples sizes $n=100,200,300,400,500,600,700,800,900$ and 1000 . The true values of the parameters are $\alpha=0.5, \beta=1$ and $\lambda=1.5$. For each combination of parameters and sample sizes, we generated 1000 random samples of the SN-BS, ST-BS $(\nu=3)$, SSL-BS $(\nu=3)$ and SCN-BS $(\nu=0.1, \gamma=0.1)$ models. To analyze asymptotic properties of the ML estimates, we computed the square root of the mean square error (RMSE), the Bias and the Monte Carlo standard deviation (MC-Sd) for each parameter over the 500 replicates. For the parameter $\theta$, these quantities are defined, respectively, by

$$
\operatorname{MC}-\operatorname{SD}(\widehat{\theta})=\sqrt{\frac{1}{m-1} \sum_{j=1}^{m}\left(\widehat{\theta}^{(j)}-\overline{\hat{\theta}}\right)^{2}} \text { and } \operatorname{RMSE}(\widehat{\theta})=\sqrt{\operatorname{MC}^{2} \operatorname{Sd}^{2}(\widehat{\theta})+\operatorname{Bias}^{2}(\widehat{\theta})}
$$

where $\operatorname{Bias}(\widehat{\theta})=\overline{\widehat{\theta}}-\theta$, with $\overline{\hat{\theta}}=\frac{1}{m} \sum_{j=1}^{m} \widehat{\theta}^{(j)}$ is the Monte Carlo mean and $\theta^{(j)}$ is the estimate of $\theta$ from the $j$-th sample, $j=1, \ldots, m$. Figure 2.3 presents the Bias and RMSE of the ML estimates of the parameters $\alpha, \beta$ and $\lambda$, from which we can note that the Bias and RMSE become smaller as the sample size $n$ increases, as one would expect. Note that estimates of the parameters are not all accurate in all considered cases for small $n$, in terms of bias, especially the ML estimates of $\lambda$.

$\alpha$
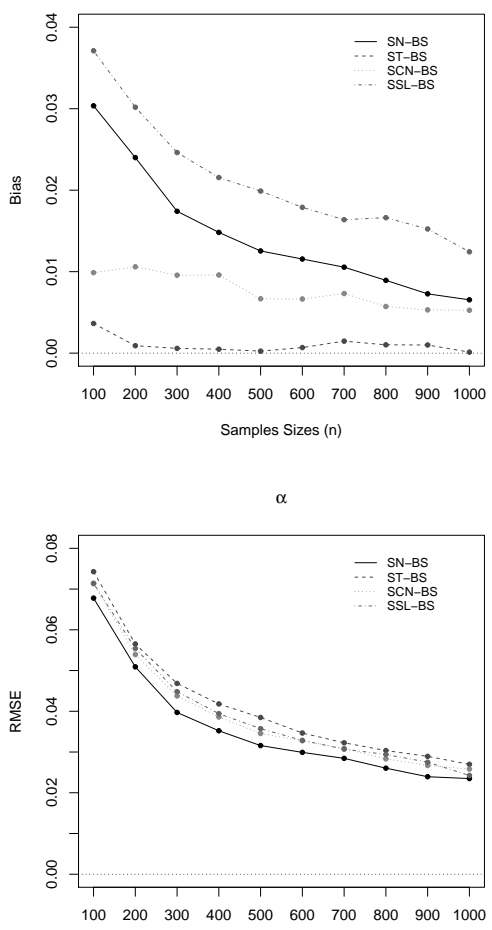

Samples Sizes (n) $\beta$

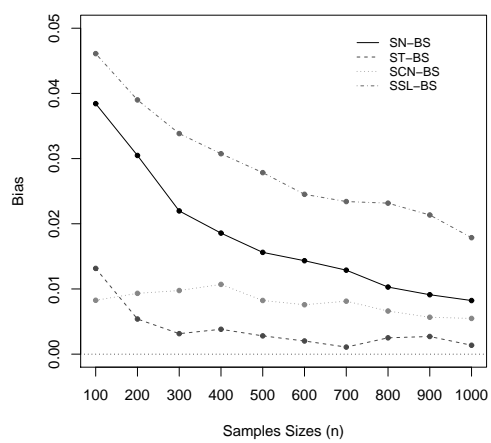

$\beta$

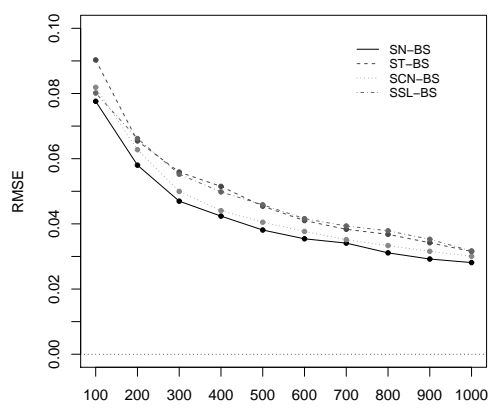

Samples Sizes (n)

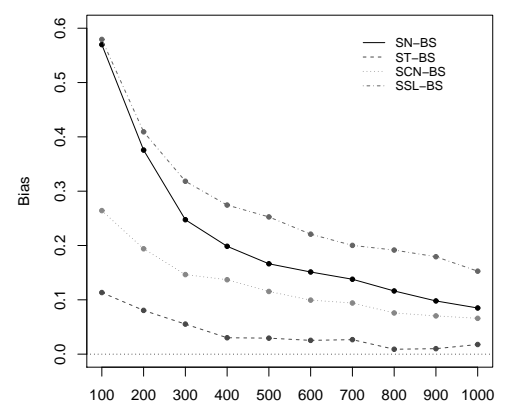

Samples Sizes (n)

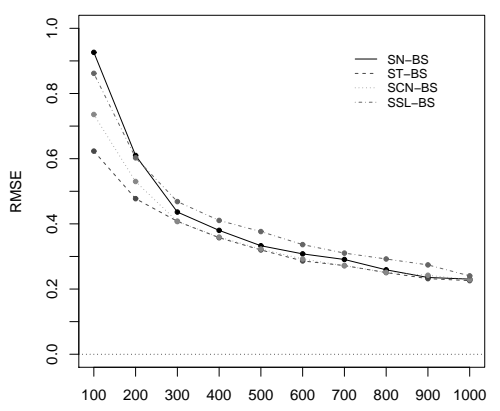

Samples Sizes (n)

Figure 2.3: Bias (upper panel) and RMSE (lower panel) of parameters $\alpha, \beta$ and $\lambda$, respectively. 


\subsubsection{Experiment 3: Robustness properties of EM estimates}

The purpose of this simulation study is to discuss robustness aspects of the SMSN-BS model, which is studied through of the influence of a single outlying observation on the ML estimates of the model parameters. We simulated one dataset from some special cases of SMSN-BS models with fixed $\nu$, considering the SN-BS, ST-BS $(\nu=3)$, SCN-BS $(\nu=0.1, \gamma=0.1)$ and SSL-BS $(\nu=3)$ models, with $\alpha=0.5, \beta=1$ and $\lambda=1.5$. For these cases, we generated 1000 samples of size $n=500$, and so we assessed how much the EM estimates are influenced in the presence of outliers, by replacing a single observation $t_{150}$ by $t_{150}=t_{150}+\vartheta$, with $\vartheta=1, \ldots, 10$. For each replication, we obtained the parameter estimates with and without outliers, in all models. We evaluated the relative change in the estimates of $\boldsymbol{\theta}=(\alpha, \beta, \lambda)^{\top}$ through

$$
R C\left(\widehat{\theta}_{i}(\vartheta)\right)=\left|\frac{\widehat{\theta}_{i}(\vartheta)-\widehat{\theta}_{i}}{\widehat{\theta}_{i}}\right|,
$$

where $\widehat{\theta}_{i}(\vartheta)$ and $\widehat{\theta}_{i}$ denote the ECM estimates of $\theta_{i}$ with and without perturbation, respectively. Figure 2.4 shows the average values of the relative changes undergone by all the parameters. We note that for all parameters, the average relative changes suddenly increase in SN-BS model as the $\vartheta$ value grows. In contrast, for the ST-BS, SSL-BS and SCN-BS models, the measures vary little, indicating they are more robust than the SN-BS model in the ability to accommodate discrepant observations.
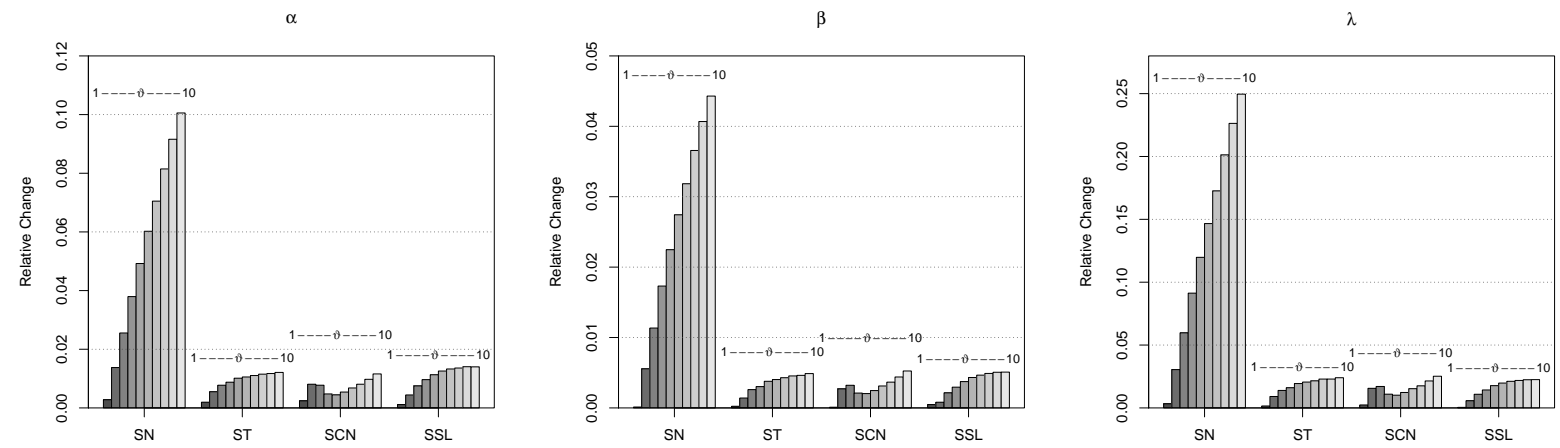

Figure 2.4: Average relative changes of estimates for different perturbations $\vartheta$

\subsection{Breaking stress dataset}

In this application, we consider a real uncensored dataset from Nichols and Padgett (2006) on breaking stress of carbon fibers (in Gba). This dataset has also been analyzed by Cordeiro et al. 
(2013) to illustrate their approach. We consider our model for the variable tensile strength, which is represented as

$$
T_{i} \sim \operatorname{SMSN-BS}(\alpha, \beta, \lambda ; G), \quad i=1, \ldots, 100
$$

The ML estimates of the five models (BS, SN-BS, ST-BS, SCN-BS and SSL-BS) are reported in Table 2.2, along with the corresponding standard errors (SE), obtained via the information-based procedure presented in Section 2.4.2. Also, we report the percentile estimates of the percentiles $t_{p}$, with $p=0.1 ; 0.25 ; 0.5 ; 0.75 ; 0.9$.

Table 2.2: Parameter estimates and SE of the SMSN-BS models and percentile estimates

\begin{tabular}{ccccccccccc}
\hline \hline \multirow{2}{*}{ Parameter } & \multicolumn{2}{c}{ BS } & \multicolumn{2}{c}{ SN-BS } & \multicolumn{2}{c}{ ST-BS } & \multicolumn{2}{c}{ SCN-BS } & \multicolumn{2}{c}{ SSL-BS } \\
\hline & ML & SE & ML & SE & ML & SE & ML & SE & ML & SE \\
\hline$\alpha$ & 0.4622 & 0.0270 & 0.7440 & 0.0625 & 0.5497 & 0.0824 & 0.1756 & 0.0140 & 0.5045 & 0.0714 \\
$\beta$ & 2.3660 & 0.1275 & 4.0943 & 0.3003 & 3.7786 & 0.3127 & 3.4357 & 0.1432 & 3.8912 & 0.3221 \\
$\lambda$ & - & - & -3.8061 & 1.5467 & -2.5337 & 1.1576 & -1.7897 & 0.6119 & -2.8920 & 1.3143 \\
$\nu$ & - & - & - & - & 5.8272 & - & 0.7003 & - & 2.0481 & - \\
$\gamma$ & - & - & - & - & - & - & 0.0599 & - & - & - \\
\hline$t_{10}(1.349)$ & 1.3195 & 1.2861 & 1.3522 & 1.2542 & & 1.3586 \\
$t_{25}(1.840)$ & 1.7345 & & 1.7823 & & 1.9007 & 1.8067 & 1.8833 \\
$t_{50}(2.700)$ & 2.3660 & 2.4923 & 2.5696 & 2.6387 & 2.5557 \\
$t_{75}(3.220)$ & 3.2274 & 3.2692 & 3.2538 & 3.2041 & 3.2669 \\
$t_{90}(3.682)$ & 4.2425 & 3.9657 & 3.8937 & 3.7024 & 3.9286 \\
\hline \hline
\end{tabular}

Table 2.3 compares the fit of members of SMSN-BS models based on symmetric (SMN, in parentheses) and asymmetric (SMSN) models, using the following model selection criteria: Akaike information criterion (AIC), Bayesian information criterion (BIC) and the efficient determination criterion (EDC). From this table, we note that all criteria indicate that the ST-BS, SCN-BS and SSL-BS models outperform the SN-BS model, indicating that distributions with heavier tails provide a better fit than the SN-BS distribution. In addition, the BS distribution based on SMSN distribution provides a better fit than the BS distribution based on the SMN distribution.

Table 2.3: Some information criteria (columns 2-4), log-likelihood (columns 5): SMSN-BS model vs corresponding SMN-BS model (in parentheses).

\begin{tabular}{ccccc}
\hline \hline Models & AIC & BIC & EDC & log-lik \\
\hline SN (N) & $292.437(304.122)$ & $300.252(309.332)$ & $292.437(304.122)$ & $-143.218(-150.061)$ \\
ST (T) & $288.361(297.116)$ & $296.176(302.326)$ & $288.361(297.116)$ & $-141.180(-146.558)$ \\
SCN (CN) & $286.372(297.149)$ & $294.188(304.965)$ & $286.372(297.149)$ & $-140.186(-145.575)$ \\
SSL (SL) & $288.550(297.295)$ & $296.366(302.505)$ & $288.550(297.295)$ & $-141.275(-146.647)$ \\
\hline \hline
\end{tabular}


Figure 2.5 shows the histogram of the Stress dataset superimposed on the fitted curves and the estimated pdf and cdf fitted distributions for BS, SN-BS and SMSN-BS densities. From these plots, we conclude that the SCN-BS models yield the best fits and that they can be adequate for these data.

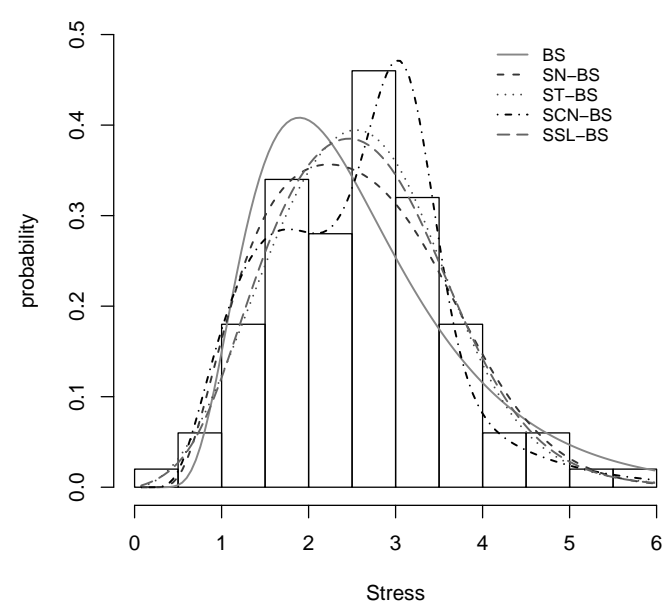

(a)

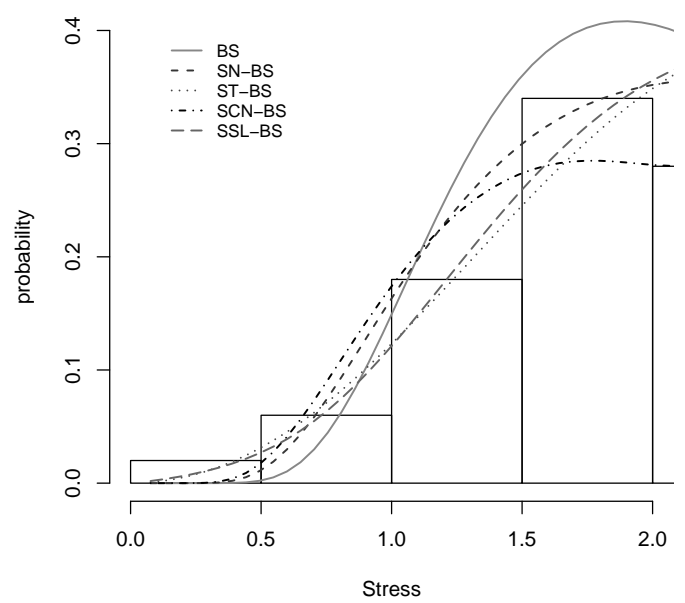

(c)

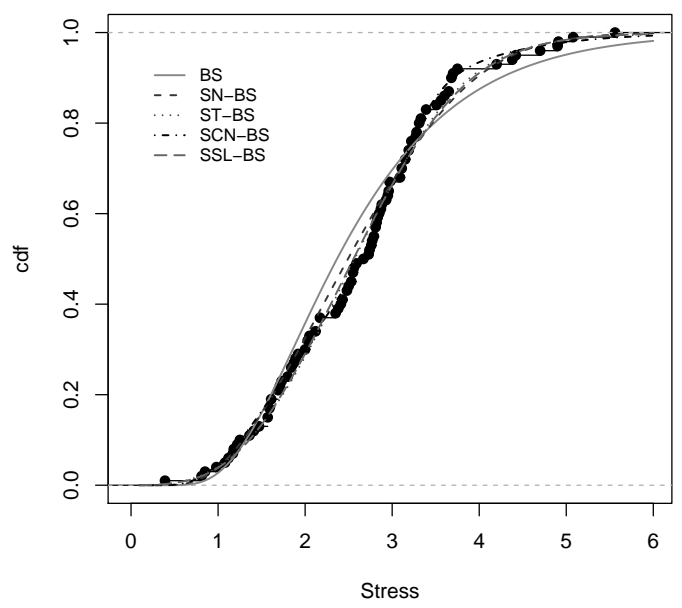

(b)

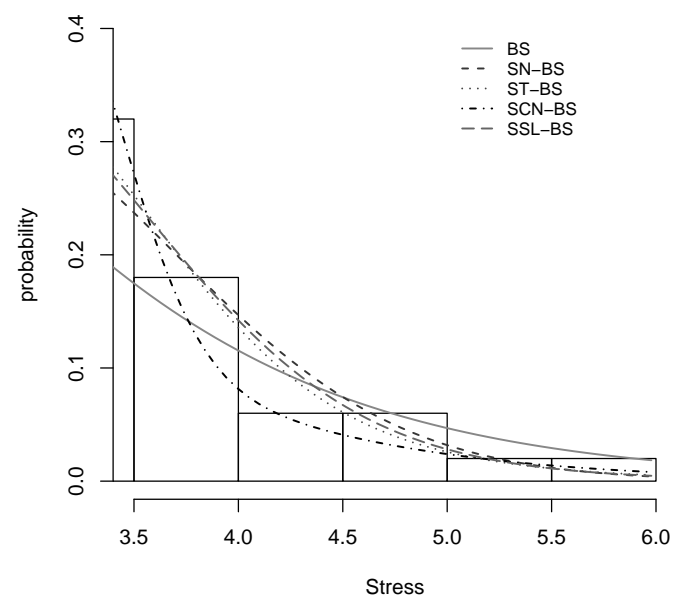

(d)

Figure 2.5: Stress. Histogram of the Stress data with five $M L$-fitted densities $B S, S N-B S$ and $S M S N-B S$ overlaid. 


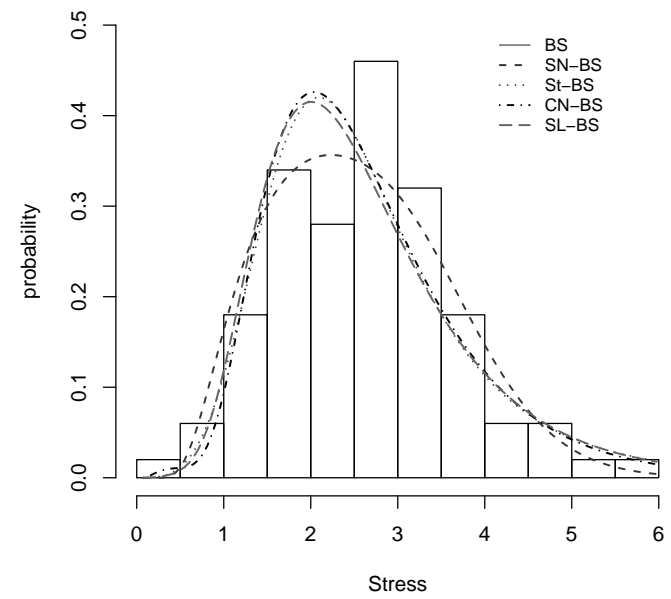

(a)

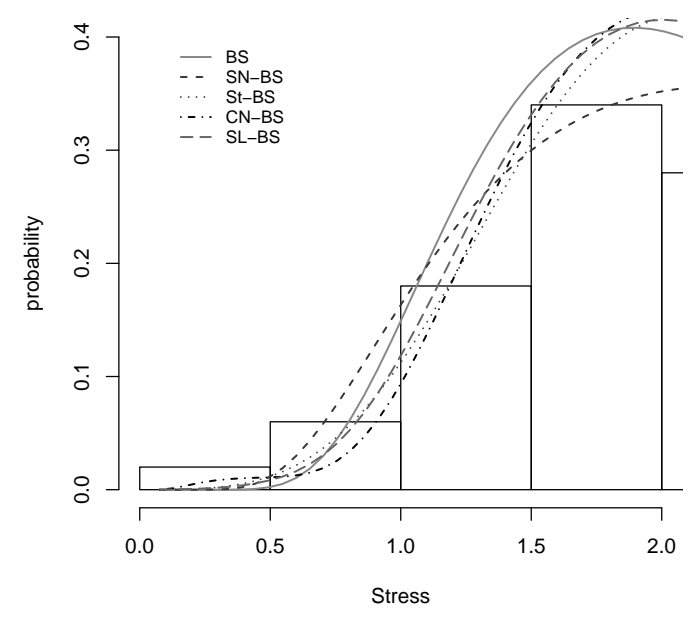

(c)

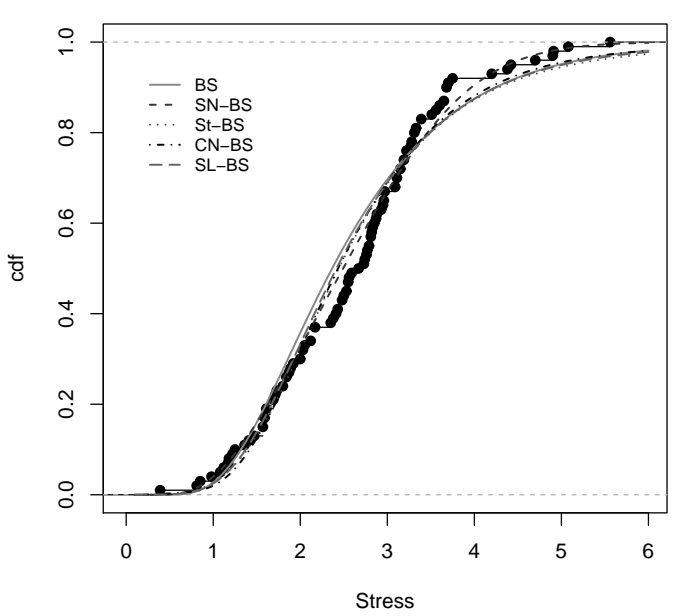

(b)

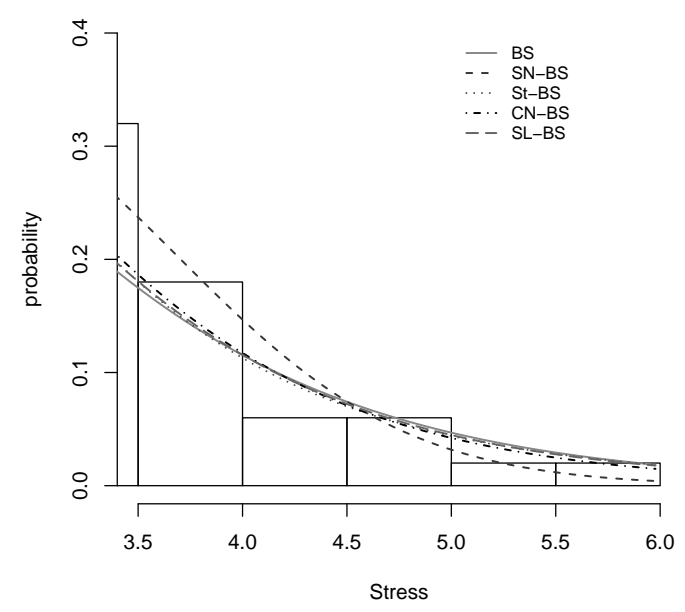

(d)

Figure 2.6: Stress. Histogram of the Stress data with five $M L$-fitted densities BS, SN-BS and SMN-BS overlaid.

Figure 2.6 shows the histogram of the Stress dataset superimposed on the fitted curves and the estimated pdf and cdf fitted distributions for BS, SN-BS and SMN-BS densities. From these plots, we conclude that the SMSN-BS models yield better fits than symmetric models. Moreover, by replacing the ML estimates of $\boldsymbol{\theta}$ in $d(T)$ we constructed the QQ-plots and envelopes by simulation for the SN-BS, SCN-BS and SSL-BS model as depicted in Figure 2.7. The estimated envelope for the SCN model indicates no points outside the confidence band (Figure 2.7(d)). Therefore, these plots enable us to claim that the SCN model provides a better fit to the data than the other models. 


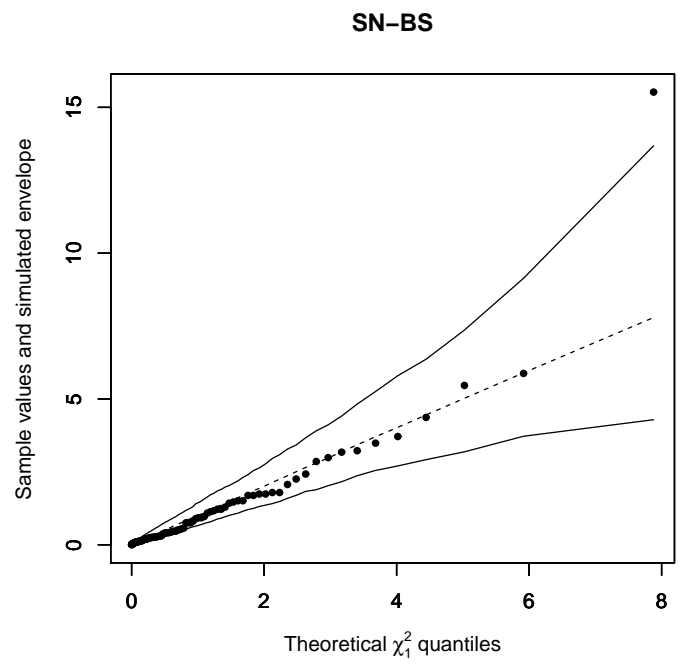

(a)

SSL-BS

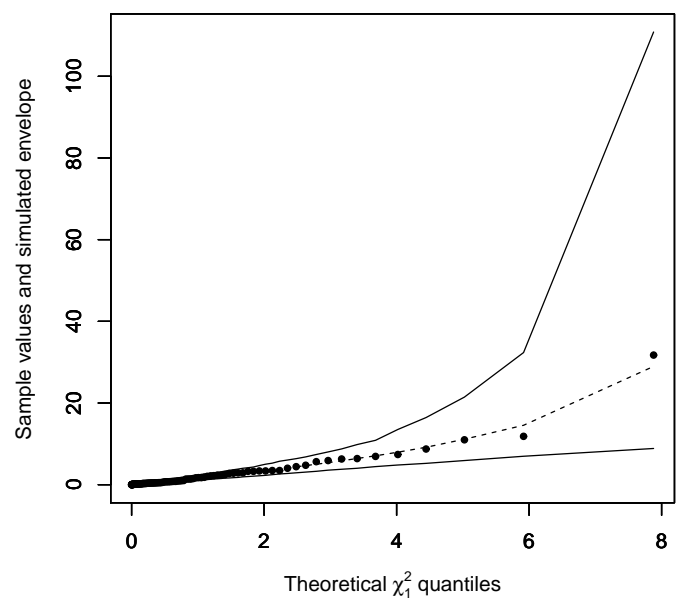

(c)

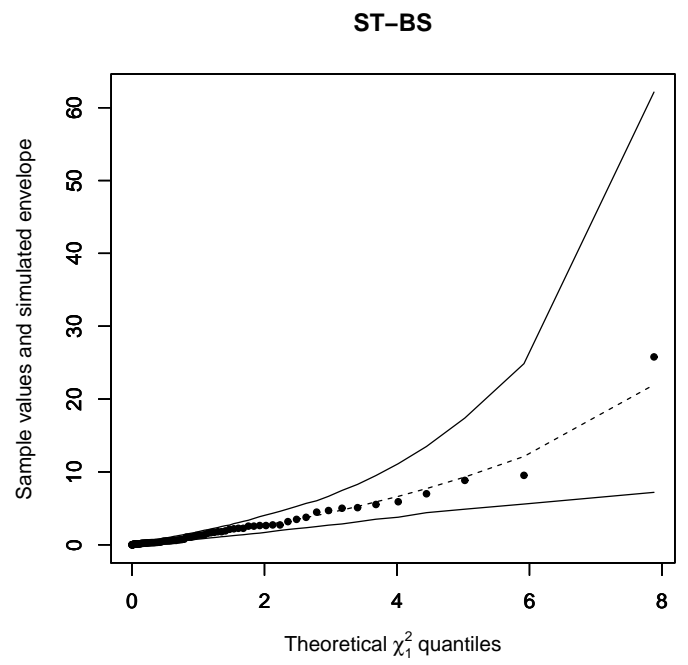

(b)

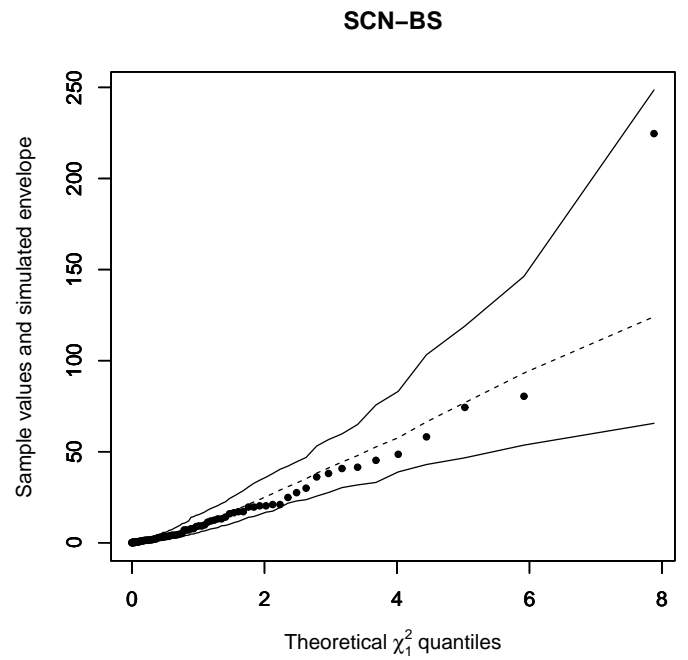

(d)

Figure 2.7: Stress. $Q-Q$ plots and simulated envelopes.

\subsection{Concluding remarks}

In this work, we have developed a new class of SMSN-BS distributions, extending the distributions proposed by Birnbaum and Saunders (1969a), Vilca et al. (2011) and Balakrishnan et al. (2009), which in turn were extensions of BS distributions based on the normal, skew-normal and scale mixtures of normal distributions, respectively. The SMSN-BS distributions have as special cases the distributions based on the skew-normal, skew-t, skew-contaminated normal, skew-slash models. So, our approach provides a robust alternative to parameter estimation in BS models. This 
class of SMSN-BS distributions allows us to predict extreme percentiles very well, especially in the left-tail. These extreme left percentiles are not easily estimated when using BS models based on symmetrical distributions. In fact, if the data follow an SMSN-BS distribution, with a negative $\lambda$ as in Figure 2.2, and we instead fit the data either by the BS model or a BS model based on a symmetric distribution, we end up overestimating the lower percentiles. Furthermore, other advantages of the classes of SMSN-BS distributions are easy of simulating data and nice hierarchical representation that facilitates the implementation of an EM algorithm for the maximum-likelihood estimation and determines the observed information matrix. We illustrated our results by using numerical examples, showing the flexibility and inherent robustness of the estimation procedure in the SMSN-BS model. 


\title{
Chapter 3
}

\section{Sinh-skew-normal/Independent}

\section{Regression Models}

\begin{abstract}
Skew-normal/independent (SNI) distributions form an attractive class of asymmetric heavytailed distributions that also accommodate skewness. We use this class of distributions here to derive a generalization of sinh-normal distributions (Rieck, 1989), called the sinh-skew-normal/independent (sinh-SNI) distribution. Based on this distribution, we then propose a general class of nonlinear regression models, generalizing the regression models of Rieck and Nedelman (1991) that have been used extensively in Birnbaum-Saunders regression models. The proposed regression models have a nice hierarchical representation that facilitates easy implementation of an EM-algorithm for the maximum likelihood estimation of model parameters and provide a robust alternative to estimation of parameters. Simulation studies as well as applications to a real dataset are presented to illustrate the usefulness of the proposed model as well as all the inferential methods developed here.
\end{abstract}

Keywords: Nonlinear regression; Birnbaum-Saunders distribution; EM-algorithm; Robust estimation; Skew-normal/Independent distribution; Sinh-normal distribution.

\subsection{Introduction}

The normal distribution has played a dominant role in both theoretical and applied statistics, but it is known not to always provide an adequate representation for many datasets in practice. According to Johnson (1949), a way to overcome this problem is to build non-normal distributions 
by transforming a normal random variable suitably. Some examples are the Birnbaum-Saunders (BS) (Birnbaum and Saunders, 1969a), sinh-normal Rieck (1989), log-normal (Johnson et al., 1994) and Johnson distributions, among several others. From a lifetime data viewpoint, the relationship between sinh-normal and Birnbaum-Saunders distributions have been discussed by Rieck (1989) and Leiva et al. (2010). In addition, Rieck and Nedelman (1991) considered that relationship in regression models, especially when the lifetime data are modeled by the distribution of Birnbaum and Saunders (1969a), in which the scale parameter depends on covariates. The BS distribution has been widely used including in engineering, industry, business and medical sciences. In a business context, Paula et al. (2011) considered a type of BS model based on the Student- $t$ distribution and applied it to insurance modeling. Lemonte and Cordeiro (2009) proposed a Birnbaum-Saunders nonlinear regression model (BS-NLM) based on the BS distribution, or simply sinh-normal nonregression models (sinh-normal-NLM), which represent a generalization of the linear regression model of Rieck and Nedelman (1991).

As the sinh-normal distribution is symmetric, it can be used in the same way as symmetric or elliptical distributions are used in building regression models. However, the sinh-normal distribution may not be suitable to represent data containing outlying observations due to its close relationship with the normal distribution. To overcome this problem, the relationship between sinh-normal and normal distributions is utilized to obtain a general class of sinh-normal distributions based on the family of scale mixtures of normal (SMN) (Andrews and Mallows, 1974) distributions, or simply normal /Independent (NI) (Lange and Sinsheimer, 1993) distribution, which contains many important unimodal and symmetric distributions such as the contaminated normal, slash, Student-t and Laplace distributions, as special cases. Generalization of the sinh-normal distribution based on the NI distributions can be found in Paula et al. (2011), Vilca et al. (2015) and Vilca et al. (2017). When the data display skewness, the skew-normal (SN) distribution of Azzalini (1985) could be used in place of the normal distribution to accommodate the tails (left or right) in a better way. This idea has been considered in the Birnbaum-Saunders distribution setting by Vilca et al. (2011) in the prediction of extreme percentiles and Santana et al. (2011) subsequently proposed a Birnbaum-Saunders linear regression model in which the normal distribution has been replaced by the skew-normal distribution. A generalization of the skew-normal distribution, following the lines of Andrews and Mallows (1974) and Lange and Sinsheimer (1993), has been proposed by Branco and Dey (2001), called scale mixtures of skew-normal (SMSN) distributions, or simply skew-normal /Independent (SNI) distributions.

The aim of this paper is to provide a generalization of the sinh-Normal ( $\sinh -\mathrm{N})$ distribution based 
on the SNI, called sinh-skew-normal/independent (sinh-SNI) distributions. This new family of distributions is attractive as it simultaneously models skewness and heavy tails. This class contains generalizations based on the skew-normal (SN), the skew-t (ST), the skew-slash (SSL), the skewcontaminated normal (SCN) distributions, and those based on symmetric class of NI distributions, called sinh-NI distribution; see Vilca et al. (2015). This generalization also results in making the inference robust against departures (for example, by the presence of few outlying observations) from the sinh-normal and sinh-SN distributions.

This family of sinh-SNI distributions is quite flexible and convenient for use in practice, and also can be used in regression setup. Inspired by Lemonte and Cordeiro (2009) and Vilca et al. (2015), who developed the Birnbaum-Saunders nonlinear regression model under sinh-N and sinh-NI distributions, respectively, we consider here an extension of that model in which the symmetric NI distribution is replaced by the asymmetric SNI distribution. The resulting model is referred to as sinh-SNI nonlinear regression model (sinh-SNI-NLM). We investigate some inferential aspects of this model and the robust estimation of parameters in a manner similar to that of the SNI model. A key feature of this regression model is that it can be formulated in a flexible hierarchical representation that is useful for some theoretical derivations such as the computation of the maximum likelihood (ML) estimates of the model parameters efficiently through an EM-algorithm (Dempster et al., 1977).

The rest of this paper is organized as follows. In Section 3.2, we briefly review sinh-normal and SNI distributions and then introduce the sinh-SNI distribution, with some of its properties that are needed in the estimation of parameters. In Section 3.3, we present the sinh-SNI nonlinear regression models. An EM-algorithm for determining the ML estimates is then presented and the observed information matrix is derived. In Sections 3.4 and 3.5, some numerical examples using both simulated and real data are given to illustrate the usefulness of the proposed models as well as the inferential methods developed here. Finally, some concluding remarks are made in Section 3.6 .

\subsection{The Sinh-SNI distribution}

First, we briefly describe the sinh-Normal distribution of Rieck (1989). A r.v. $Y$ is said to have a sinh-Normal distribution if it is related to the standard normal distribution through the stochastic representation $Y=\mu+\sigma \operatorname{arcsinh}\left(\alpha Z_{0} / 2\right)$, where $Z_{0} \sim \mathrm{N}(0,1)$. Following Balakrishnan et al. (2009) and Vilca et al. (2017), we extend this sinh-normal distribution by replacing the standard normal 
distribution by a standard SNI distribution. Recall that a random variable $Z$ is said to have a standard SNI distribution if it is related to the skew-normal distribution through the stochastic representation

$$
Z=U^{-1 / 2} Z_{0}
$$

where $Z_{0}$ follows a standard skew-normal distribution, denoted by $Z_{0} \sim \mathrm{S} N(0,1, \lambda)$, and $U$ is a positive r.v. independent of $Z_{0}$. It is well-known that $Z_{0}$ can be represented stochastically as $Z_{0}=\delta H+X_{1} \sqrt{1-\delta^{2}}$, where $H=\left|X_{0}\right|$ and $X_{1}$ are independent, and they have half-normal and normal distributions, respectively. So, a r.v. $Y$ stochastically represented in terms of $Z=$ $\delta U^{-1 / 2} H+\sqrt{1-\delta^{2}} U^{-1 / 2} X_{1}$ by

$$
Y=\mu+\sigma \operatorname{arcsinh}\left(\frac{\alpha Z}{2}\right)=\mu+\sigma \operatorname{arcsinh}\left(\frac{\alpha}{2}\left[\delta U^{-1 / 2} H+\sqrt{1-\delta^{2}} U^{-1 / 2} X_{1}\right]\right)
$$

is said to have a sinh-SNI distribution with shape parameter $\alpha>0$, location parameter $\mu \in \mathbb{R}$, scale parameter $\sigma>0$ and skewness parameter $\lambda \in \mathbb{R}$. The notation $Y \sim \operatorname{Sinh}-\operatorname{SNI}(\alpha, \mu, \sigma, \lambda ; G)$ is used for this distribution, where $G($.$) is the cumulative distribution function (cdf) of U$ (indexed by a scalar or vector parameter $\boldsymbol{\nu}$ ). Some special cases of this distribution are as follows. When $\lambda=0$, the distribution reduces to the distribution discussed by Balakrishnan et al. (2009) and Vilca et al. (2017) which extends the sinh-normal distribution upon replacing the standard normal distribution by a SNI distribution. When $U=1$, the SNI distribution reduces to the skewed sinhnormal distribution (Leiva et al., 2010). Moreover, when $\sigma=2$, the r.v. $T=\exp (Y)$ follows a scale mixture of skew-normal Birnbaum-Saunders (SNI-BS) distribution recently discussed by Maehara et al. (2017).

The stochastic representation in (3.2) is useful for generating random numbers from the sinhSNI distribution and also in deriving their structural properties and in implementing the EMalgorithm for calculating the maximum likelihood estimates of the model parameters. Moreover, from the stochastic representation, we observe that the conditional distribution of $Y$, given $U=u$, follows the sinh-skew-normal (sinh-SN) distribution, i.e., $Y \mid(U=u) \sim \operatorname{Sinh}-\operatorname{SN}\left(\alpha_{u}, \mu, \sigma, \lambda\right)$, where $\alpha_{u}=u^{-1 / 2} \alpha$. From this fact, the probability density function (pdf) of $Y$ can be expressed as

$$
f_{Y}(y)=\frac{1}{\sigma} \phi_{\mathrm{SNI}}\left(\xi_{2 y}\right) \xi_{1 y}=\frac{1}{\sigma} \int_{0}^{\infty} \phi\left(\xi_{2 y} ; 0, u^{-1}\right) \Phi\left(\lambda u^{1 / 2} \xi_{2 y}\right) d G(u) \xi_{1 y}, y \in \mathbb{R}
$$

where $\phi_{\mathrm{SNI}}(\cdot)$ is the pdf of $Z$ in $(3.1), \xi_{1 y}=\xi_{1}(y ; \alpha, \mu, \sigma)=\frac{2}{\alpha} \cosh \left(\frac{y-\mu}{\sigma}\right), \xi_{2 y}=\xi_{2}(y ; \alpha, \mu, \sigma)$ $=\frac{2}{\alpha} \sinh \left(\frac{y-\mu}{\sigma}\right), \phi\left(\psi, \eta^{2}\right)$ denotes the $N\left(\psi, \eta^{2}\right)$ density function, and $\Phi(\cdot)$ is the cdf of the standard 
normal distribution. It can be shown easily that if $Y \sim \sinh -\operatorname{SNI}(\alpha, \mu, \sigma, \lambda ; G)$, then $W=a+b Y \sim$ sinh-SNI $(\alpha, a+b \mu,|b| \sigma, \lambda ; G)$, where $a \in \mathbb{R}$ and $b \neq 0$. This result implies that the sinh-SNI family is closed under linear transformation. The moment generating function (mgf) of $Y$ can be expressed as

$$
M_{Y}(s)=\exp (\gamma s) \int_{0}^{\infty}\left[\frac{K_{(2 s+1) / 2}\left(\alpha_{u}^{-2} ; \lambda\right)+K_{(2 s-1) / 2}\left(\alpha_{u}^{-2} ; \lambda\right)}{K_{1 / 2}\left(\alpha_{u}^{-2}\right)}\right] d G(u), \mathrm{s} \in \mathbb{R}
$$

where $\alpha_{u}=u^{-1 / 2} \alpha$ and

$$
K_{\nu}(w ; \lambda)=\frac{1}{2} \int_{-\infty}^{\infty} \exp (-w \cosh (u)-\nu u) \Phi\left(\frac{2 \lambda}{\alpha} \sinh \left(\frac{u}{2}\right)\right) \mathrm{d} u
$$

which satisfies $K_{\nu}(w ; \lambda=0)=K_{\nu}(w) / 2$, with $K_{\nu}(\cdot)$ denoting the modified Bessel function of the third kind defined by

$$
K_{\nu}(w)=\frac{1}{2} \int_{-\infty}^{\infty} \exp (-w \cosh (u)-\nu u) \mathrm{d} u
$$

Thus, the moments of $Y$ may be approximated for some special cases.

Theorem 5. Let $Y \sim \operatorname{Sinh}-\operatorname{SNI}(\alpha, \mu, \sigma, \lambda ; G)$ and $H \mid(U=u) \sim \operatorname{HN}\left(0, u^{-1}\right)$. Then, the conditional distribution of $Y$, given $H=h$ and $U=u$, has its pdf as

$$
f_{Y \mid H, U}(y \mid h, u)=\phi\left(\lambda_{h, u}+\xi_{2}\left(y ; \alpha_{\delta, u}, \mu, \sigma\right)\right) \xi_{1}\left(y ; \alpha_{\delta, u}, \mu, \sigma\right), \quad y \in \mathbb{R}
$$

where $\alpha_{\delta, u}=u^{1 / 2} \alpha \sqrt{1-\delta^{2}}, \lambda_{h, u}=-u^{1 / 2} \delta h / \sqrt{1-\delta^{2}}, \xi_{2}\left(y ; \alpha_{\delta, u}, \mu, \sigma\right)$ and $\xi_{1}\left(y ; \alpha_{\delta, u}, \mu, \sigma\right)$ are as given in (3.3) with $\alpha_{\delta, u}$ instead of $\alpha$, and $\delta=\lambda / \sqrt{1+\lambda^{2}}$.

The conditional distribution of $Y$, given $H=h$ and $U=u$, is the four-parameter SHN distribution proposed by Leiva et al. (2010), denoted by $Y \mid(H=h, U=u) \sim \operatorname{SHN}\left(\alpha_{\delta, u}, \mu, \sigma=2, \lambda_{h, u}\right)$. This will be used later in the development of EM-algorithm for determining the maximum likelihood estimates of the model parameters.

Theorem 6. Let $Y \sim \operatorname{Sinh}-\operatorname{SNI}(\alpha, \mu, \sigma, \lambda ; H)$. Then, $H \mid(Y=y, U=u)$ has a standard $H N$ distribution with $p d f$

$$
f_{H \mid Y, U}(h \mid y, u)=\frac{\phi\left(h ; \delta \xi_{2}(y ; \alpha, \mu, \sigma), u^{-1}\left(1-\delta^{2}\right)\right)}{\Phi\left(\lambda \xi_{2}\left(y ; \alpha_{u}, \mu, \sigma\right)\right)}, \quad h>0,
$$


where $\alpha_{u}=\alpha u^{-1 / 2}$. Moreover,

$$
\begin{aligned}
\mathrm{E}[U H \mid Y=y] & =\delta \xi_{2}(y ; \alpha, \mu, \sigma) \kappa_{y}+\tau_{y} \sqrt{1-\delta^{2}} \\
\mathrm{E}\left[U H^{2} \mid Y=y\right] & =\delta^{2} \xi_{2}^{2}(y ; \alpha, \mu, \sigma) \kappa_{y}+\left[1-\delta^{2}\right]+\delta \xi_{2}(y ; \alpha, \mu, \sigma) \tau_{y} \sqrt{1-\delta^{2}}
\end{aligned}
$$

where $\kappa_{y}=\mathrm{E}[U \mid Y=y]$ and $\tau_{y}=\mathrm{E}\left[U^{1 / 2} W_{\Phi}\left(\frac{\delta \xi_{2}\left(y ; \alpha_{U}, \mu, \sigma\right)}{\sqrt{1-\delta^{2}}}\right) \mid Y=y\right]$, with $W_{\Phi}(u)=\phi(u) / \Phi(u)$ for $u \in \mathbb{R}$.

Proof. Following the idea of Lachos et al. (2010a), by using conditional expectation properties, we have $\mathrm{E}[U H \mid Y=y]=\mathrm{E}[U \mathrm{E}(H \mid Y, U) \mid Y=y]$ and $\mathrm{E}\left[U H^{2} \mid Y=y\right]=\mathrm{E}\left[U \mathrm{E}\left(H^{2} \mid Y, U\right) \mid Y=y\right]$. These conditional expectations depend on the conditional distribution of $H$, given $Y$ and $U$. Thus, we have

$$
\begin{aligned}
\mathrm{E}[U H \mid Y=y]= & \delta \xi_{2}(y ; \alpha, \mu, \sigma) \mathrm{E}[U \mid Y=y]+\sqrt{1-\delta^{2}} \mathrm{E}[g(U, Y) \mid Y=y], \\
\mathrm{E}\left[U H^{2} \mid Y=y\right]= & \left(\delta \xi_{2}(y ; \alpha, \mu, \sigma)\right)^{2} \mathrm{E}[U \mid Y=y]+1-\delta^{2} \\
& +\delta \xi_{2}(y ; \alpha, \mu, \sigma) \sqrt{1-\delta^{2}} \mathrm{E}[g(U, Y) \mid Y=y],
\end{aligned}
$$

where $g(u, y)=u^{1 / 2} W_{\Phi}\left(\frac{\delta \xi_{2}\left(y ; \alpha_{u}, \mu, \sigma\right)}{\sqrt{1-\delta^{2}}}\right)$.

Remark 3. Let $Y \sim \operatorname{Sinh}-\operatorname{SNI}(\alpha, \mu, \sigma, \lambda ; G)$. Then:

i) If $\Phi_{\mathrm{SNI}}(. ; \lambda)$ is the cdf of a standard SNI distribution, then the $c d f$ of $Y$ is given by $F_{Y}(y ; \alpha, \mu, \sigma, \lambda)=$ $\Phi_{\mathrm{SNI}}\left(\xi_{2}(y ; \alpha, \mu, \sigma) ; \lambda\right)$. Moreover,

(a) $F_{Y}(y ; \alpha, \mu, \sigma,-\lambda)=2 \Phi_{\mathrm{NI}}\left(\xi_{2}(y ; \alpha, \mu, \sigma)\right)-\Phi_{\mathrm{SNI}}\left(\xi_{2}(y ; \alpha, \mu, \sigma) ; \lambda\right)$, where $\Phi_{\mathrm{NI}}($.$) is the c d f$ of a standard NI distribution;

(b) $F_{Y}(y ; \alpha, \mu, \sigma, \lambda=1)=\Phi_{\mathrm{NI}}^{2}\left(\xi_{2}(y ; \alpha, \mu, \sigma)\right)$;

(c) Let $S \sim \operatorname{Sinh}-\operatorname{SNI}(\alpha, \mu, \sigma, \lambda ; G)$ be independent of $Y$. Then, $V=\max (Y, S)$ has its density as

$$
f_{V}(v)=\frac{2}{\sigma} \phi_{\mathrm{SNI}}\left(\xi_{2}(v ; \alpha, \mu, \sigma)\right) \Phi_{\mathrm{SNI}}\left(\xi_{2}(v ; \alpha, \mu, \sigma)\right) \xi_{1}(v ; \alpha, \mu, \sigma)
$$

(ii) If there is $\boldsymbol{\nu}_{\infty}$ such that $\lim _{\boldsymbol{\nu} \rightarrow \boldsymbol{\nu}_{\infty}} \phi_{\mathrm{SNI}}(z ; \lambda)=f_{0}(z ; \lambda)$ for all $z$, where $f_{0}(. ; \lambda)$ is the pdf of the $\operatorname{SN}(0,1, \lambda)$ distribution, then $\lim _{\boldsymbol{\nu} \rightarrow \boldsymbol{\nu}_{\infty}} f_{Y}(y)=f_{S N}(y ; \lambda)$ for all $y \in \mathbb{R}$, where $f_{S N}(. ; \lambda)$ is the pdf of a $\operatorname{Sinh}-\mathrm{SN}(\alpha, \mu, \sigma, \lambda)$ distribution; 
(iii) The distribution of $d(Y)=\xi_{2}^{2}(Y ; \alpha, \mu, \sigma)$ does not depend on $\lambda$. The random variable $d(Y)$ is quite useful for testing the goodness of fit of the model as well as for detecting outliers in data; see Lange and Sinsheimer (1993).

\subsubsection{Special cases of the Sinh-SNI family}

Some special cases of the sinh-SNI family are based on the skew-normal (SN), the skewcontaminated normal (SCN), skew-slash (SSL) and skew-Student- $t$ (ST) models. The resulting distributions are referred to as

(i) the sinh-skew normal (sinh-SN),

(ii) sinh-skew-contaminated normal (sinh-SCN),

(iii) sinh-skew-slash (sinh-SSL),

(iv) sinh-skew-Student- $t$ ( $\sinh -\mathrm{ST})$

distributions, respectively. Inspired by the works of Balakrishnan et al. (2009) and Vilca et al. (2017), the special cases are reported in Appendix A. Plots of the pdf are given in Figures 3.1 and
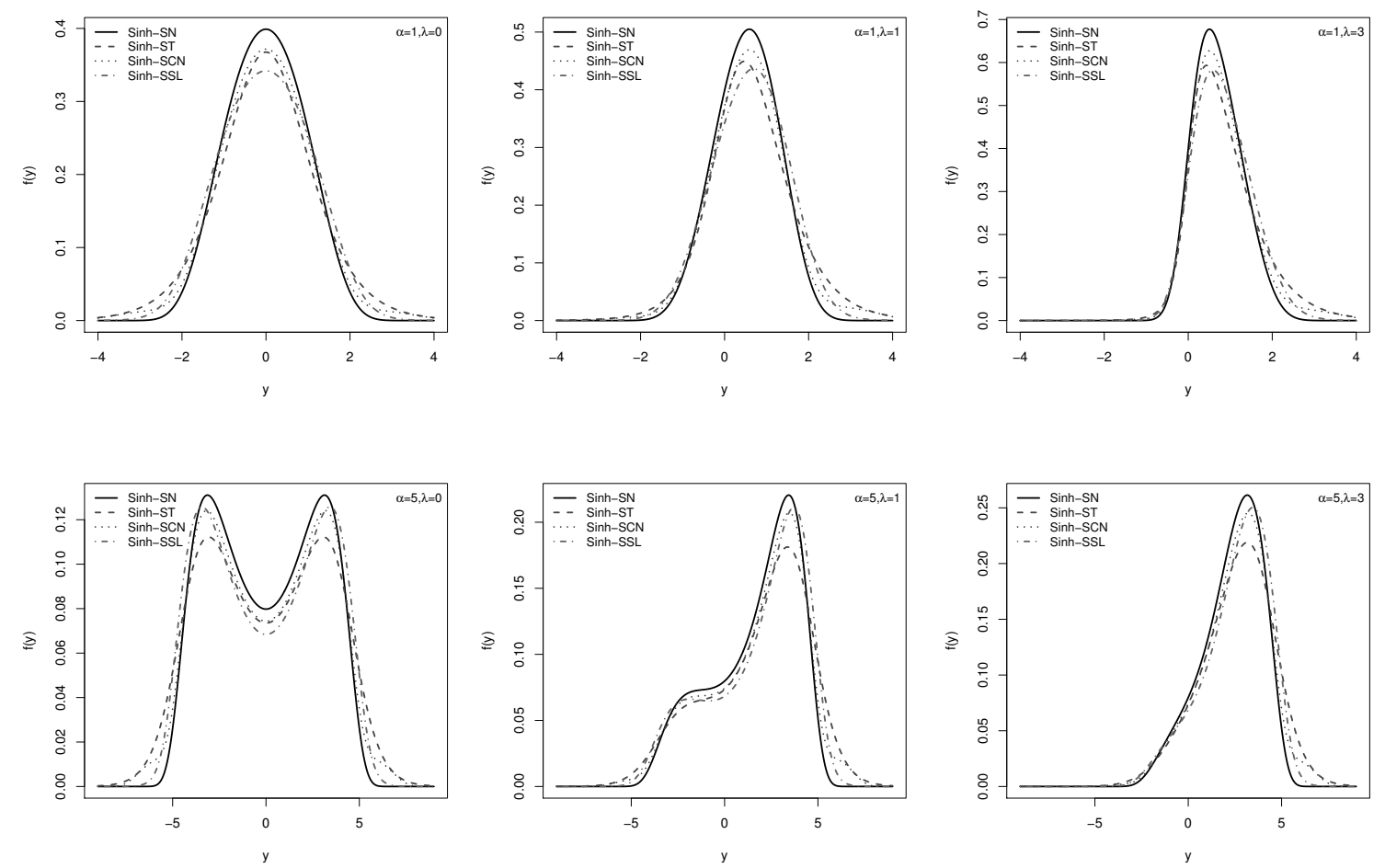

Figure 3.1: Density plots of the sinh-SNI distributions based on SN SCN, SSL and ST distributions for $\alpha=1$ (left) and $\alpha=5$ (right), respectively, when $\lambda=0,1,3$.

3.2 for values of the shape parameters $\alpha=1,5$ and the values of the parameters $\lambda=0, \pm 1, \pm 3$, 

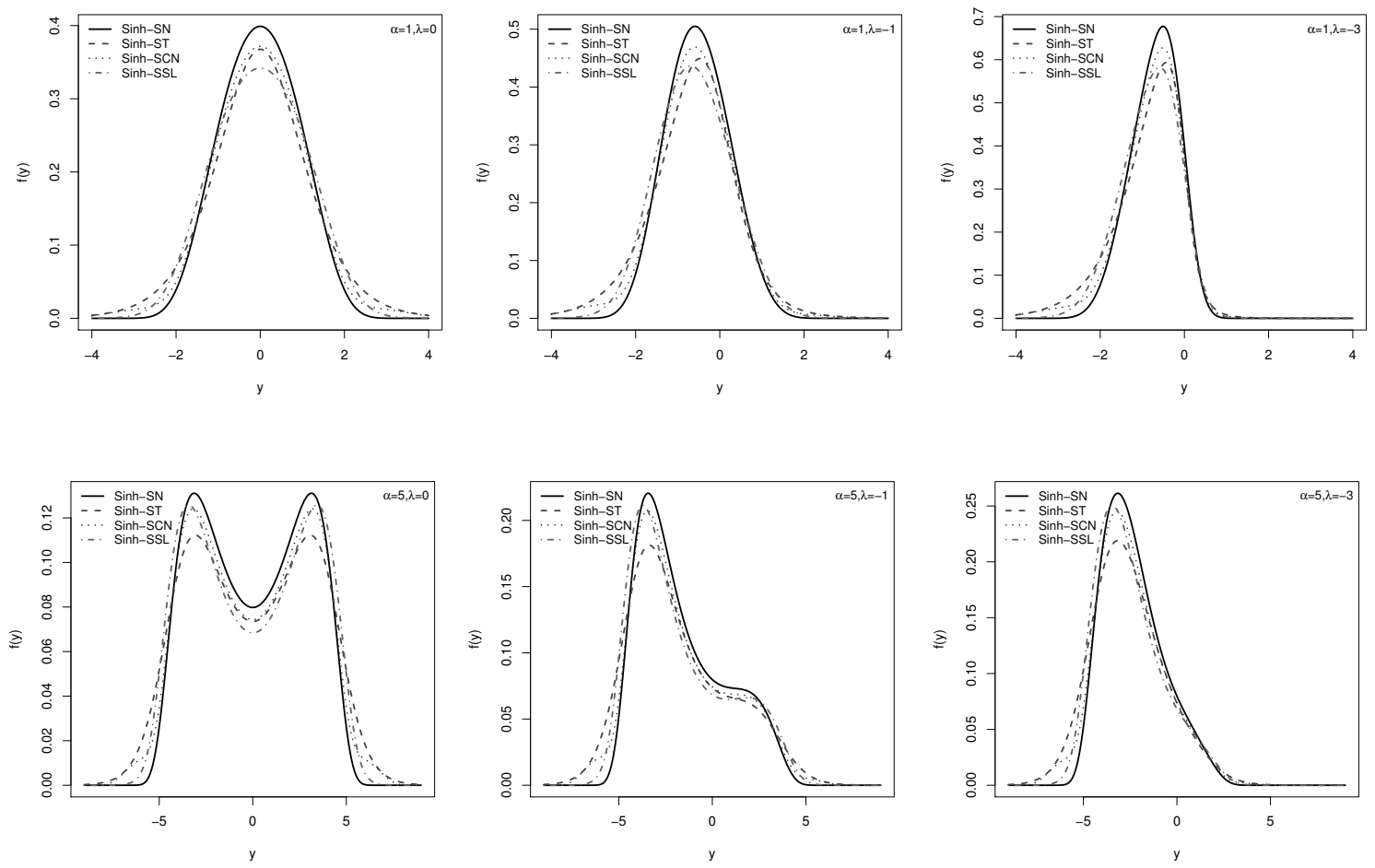

Figure 3.2: Density plots of the sinh-SNI distributions based on SN, SCN, SSL and ST distributions for $\alpha=1$ (first line) and $\alpha=5$ (second line), respectively, when $\lambda=0,-1,-3$.

with $\beta=1$ in all cases. These plots show the great flexibility that the new distribution possesses, and hence it can be very useful in modeling data revealing different shapes. For $\lambda=0$, we get symmetric distributions, and for $\lambda \neq 0$ asymmetric distributions; it can also exhibit bi-modality when $\alpha>2$ and $|\lambda|$ is close to one, but when $\lambda$ increases the density becomes unimodal. As in Rieck and Nedelman (1991), we will next use the class of sinh-SNI distributions to develop a flexible regression model.

\subsection{The proposed regression model}

In this section, we introduce the sinh-SNI regression model (sinh-SNI-RM) following the ideas of Lemonte and Cordeiro (2009) and Vilca et al. (2017). Consider the regression model

$$
Y_{i}=f\left(\boldsymbol{\beta} ; \mathbf{x}_{i}\right)+\varepsilon_{i}, i=1, \ldots, n,
$$

where $Y_{i}$ is the response variable, $\mathbf{x}_{i}$ is an $m \times 1$ vector of known explanatory variables associated with the $i$ th observable response $Y_{i}$, and $\mu_{i}(\boldsymbol{\beta})=f\left(\boldsymbol{\beta} ; \mathbf{x}_{i}\right)$ is an injective and twice differentiable function with $\boldsymbol{\beta}=\left(\beta_{1}, \ldots, \beta_{p}\right)$ being a vector of unknown nonlinear parameters. We assume that 
the errors

$$
\varepsilon_{i} \sim \operatorname{Sinh}-\operatorname{SNI}(\alpha, 0, \sigma=2, \lambda ; G), i=1, \ldots, n
$$

are independent. So, $Y_{i} \sim \operatorname{Sinh}-\operatorname{SNI}\left(\alpha, f\left(\boldsymbol{\beta} ; \mathbf{x}_{i}\right), \sigma=2, \lambda ; G\right)$ with pdf

$$
f_{Y_{i}}\left(y_{i}\right)=\frac{1}{2} \phi_{\mathrm{SNI}}\left(\xi_{2 i}\right) \xi_{1 i}
$$

where $\xi_{1 i}=\xi_{1}\left(y_{i} ; \alpha, \boldsymbol{\beta}\right)$ and $\xi_{2 i}=\xi_{2}\left(y_{i} ; \alpha, \boldsymbol{\beta}\right)$ are as in $(3.3)$, with $\sigma=2$ and $\mu_{i}=f\left(\boldsymbol{\beta} ; \mathbf{x}_{i}\right)$ (here, we use $\xi_{1 i}$ and $\xi_{2 i}$ instead of $\xi_{1 y_{i}}$ and $\xi_{2 y_{i}}$, respectively). Some special cases of the proposed regression model are as follows:

(i) for $U=1$ and $\lambda=0$, we obtain the nonlinear regression model of Lemonte and Cordeiro (2009);

(ii) for $U=1$ and $\mu_{i}=\mathbf{x}_{i}^{\top} \boldsymbol{\beta}$, we obtain the skew-BS log-linear regression model proposed by Santana et al. (2011);

(iii) for $\lambda=0$, we obtain the nonlinear regression model proposed by Vilca et al. (2015) and Vilca et al. (2017);

(iv) for $\lambda=0, U \sim \operatorname{Gamma}(\nu / 2, \nu / 2)$ and $\mu_{i}=\mathbf{x}_{i}^{\top} \boldsymbol{\beta}$, we obtain the linear regression model of Paula et al. (2011).

\subsubsection{ML estimation using an EM-algorithm}

The well-known EM algorithm is a useful tool for finding maximum likelihood (ML) estimates in incomplete-data problems. Each iteration of the EM algorithm has two steps: the expectation step (E-step), wherein we calculate the conditional expectation of the complete-data log-likelihood given the observed data ( $Q$-function) and the (current) parameter estimate, and the maximization step (M-step), wherein the (next) parameter estimate is found by maximization of the $Q$-function. One may refer to Dempster et al. (1977), for example. This maximization method is considered in the sinh-SNI-RM for finding the ML estimates of model parameters. Thus, by using the results in Section 3.2, Theorem 5 and the representation in (3.2), the regression model can be expressed in a 
hierarchical form as

$$
\begin{aligned}
Y_{i} \mid\left(H_{i}=h_{i}, U_{i}=u_{i}\right) & \stackrel{\operatorname{ind}}{\sim} \sim \operatorname{SHN}\left(\alpha_{\delta, u_{i}}, f\left(\boldsymbol{\beta} ; \mathbf{x}_{i}\right), \sigma=2, \lambda_{h_{i}, u_{i}}\right), \\
H_{i} \mid U_{i}=u_{i} & \stackrel{\text { ind }}{\sim} \sim H N\left(0, u_{i}^{-1}\right), \\
U_{i} & \stackrel{\text { ind }}{\sim} h_{U}\left(u_{i}\right), \quad i=1, \ldots, n,
\end{aligned}
$$

where $\alpha_{\delta, u_{i}}=u_{i}^{1 / 2} \alpha \sqrt{1-\delta^{2}}$ and $\lambda_{h_{i}, u_{i}}=-u_{i}^{1 / 2} \delta h_{i} /\left(\sqrt{1-\delta^{2}}\right)$. We assume that the parameter vector $\boldsymbol{\nu}$ that indexes the pdf $h_{U}(\cdot)$ is known. An optimal value of $\boldsymbol{\nu}$ can then be chosen by using the Schwarz information criterion; see Spiegelhalter et al. (2002) and Lange et al. (1989).

Let $\mathbf{u}=\left(u_{1}, \ldots, u_{n}\right)^{\top}$ be the unobserved data. Then, the complete data is $\mathbf{y}_{c}=\left(\mathbf{y}^{\top}, \mathbf{u}^{\top}\right)^{\top}$ which correspond to the original data $\mathbf{y}=\left(y_{1}, \ldots, y_{n}\right)^{\top}$ augmented with $\mathbf{u}$. Thus, under the hierarchical representation given in equations (3.8)-(3.10), it follows that the complete-data log-likelihood function for $\boldsymbol{\theta}=\left(\alpha, \boldsymbol{\beta}^{\top}, \lambda\right)^{\top}$, given $\mathbf{y}_{c}$ (without the additive constant), can be expressed as

$$
\ell_{c}\left(\boldsymbol{\theta} \mid \mathbf{y}_{c}\right)=-\frac{n}{2} \log \left(1-\delta^{2}\right)+\sum_{i=1}^{n} \log \left(\xi_{1 i}\right)-\frac{1}{2\left(1-\delta^{2}\right)} \sum_{i=1}^{n} u_{i}\left(\xi_{2 i}-\delta h_{u_{i}}\right)^{2}
$$

where $\xi_{1 i}$ and $\xi_{2 i}$ are as in (3.7). The conditional expectation of the complete-data log-likelihood function has the form

$$
\begin{aligned}
Q\left(\boldsymbol{\theta} \mid \widehat{\boldsymbol{\theta}}^{(k)}\right)= & -\frac{n}{2} \log \left(1-\delta^{2}\right)+\sum_{i=1}^{n} \log \left(\xi_{1 i}^{(k)}\right)-\frac{1}{2\left(1-\delta^{2}\right)} \sum_{i=1}^{n} \widehat{\kappa}_{i}^{(k)} \xi_{2 i}^{2(k)} \\
& +\frac{\delta}{1-\delta^{2}} \sum_{i=1}^{n} \widehat{h}_{u_{i}}^{(k)} \xi_{2 i}^{2(k)}-\frac{\delta^{2}}{2\left(1-\delta^{2}\right)} \sum_{i=1}^{n}{\widehat{h^{2}}}_{u_{i}}^{(k)},
\end{aligned}
$$

where the expressions $\widehat{\kappa}_{i}=\mathrm{E}\left[U_{i} \mid \widehat{\boldsymbol{\theta}}^{(k)}, y_{i}\right], \widehat{h}_{u_{i}}=\mathrm{E}\left[U_{i} H_{i} \mid \widehat{\boldsymbol{\theta}}^{(k)}, y_{i}\right]$ and $\widehat{h_{u_{i}}^{2}}=\mathrm{E}\left[U_{i} H_{i}^{2} \mid \widehat{\boldsymbol{\theta}}^{(k)}, y_{i}\right], i=$ $1, \ldots, n$, are obtained from Theorem 6 and $\widehat{\boldsymbol{\theta}}^{(k)}=\left(\widehat{\alpha}^{(k)}, \widehat{\boldsymbol{\beta}}^{(k)}, \widehat{\lambda}^{(k)}\right)^{\top}$ denotes the estimate of $\boldsymbol{\theta}$ at the $k$-th iteration. The quantities $\widehat{h}_{u_{i}}$ and $\widehat{h_{u_{i}}^{2}}$ are given by

$$
\begin{aligned}
\widehat{h}_{u_{i}} & =\widehat{\delta} \widehat{\xi}_{2 i} \widehat{\kappa}_{i}+\widehat{\tau}_{i} \sqrt{1-\widehat{\delta}^{2}} \\
\widehat{h}^{2} u_{i} & =\widehat{\delta}^{2} \widehat{\xi}_{2 i}^{2} \widehat{\kappa}_{i}+\left[1-\widehat{\delta}^{2}\right]+\widehat{\delta} \widehat{\xi}_{2 i} \widehat{\tau}_{i} \sqrt{1-\widehat{\delta}^{2}}
\end{aligned}
$$

and $\widehat{\kappa}_{i}$ depends on the distribution of $U$. Thus, we have the following ECM algorithm:

E-step. Given $\boldsymbol{\theta}=\widehat{\boldsymbol{\theta}}^{(k)}$, compute $\widehat{\kappa}_{i}^{(k)}, \widehat{h}_{u_{i}}^{(k)}$ and ${\widehat{h^{2}}}_{u_{i}}^{(k)}$, for $i=1, \ldots, n$, using the expressions in (3.13) and (3.14); 
CM-step 1. Fix $\widehat{\boldsymbol{\beta}}^{(k)}$ and update $\widehat{\alpha}^{(k)}$ and $\widehat{\delta}^{(k)}$ as

$$
\begin{aligned}
{\widehat{\alpha^{2}}}^{(k+1)} & =\frac{1}{n} \sum_{i=1}^{n} \widehat{\kappa}_{i} \xi_{2 i}^{2}\left(y_{i} ; 1, \widehat{\boldsymbol{\beta}}^{(k)}\right)+\left[1-\widehat{\tau}_{h_{u_{i}}}^{(k)}\right]\left[\frac{\sum_{i=1}^{n} \widehat{h}_{u_{i}}^{(k)} \xi_{2 i}\left(y_{i} ; 1, \widehat{\boldsymbol{\beta}}^{(k)}\right)}{n \widehat{\tau}_{h_{u_{i}}}^{(k)}}\right]^{2}, \\
\widehat{\delta}^{(k+1)} & =\frac{1}{\widehat{\alpha}^{(k+1)}} \frac{\sum_{i=1}^{n} \widehat{h}_{u_{i}}^{(k)} \xi_{2 i}\left(y_{i} ; 1, \widehat{\boldsymbol{\beta}}^{(k)}\right)}{n \widehat{\tau}_{h_{u_{i}}}^{(k)}}
\end{aligned}
$$

where $\xi_{2 i}\left(y_{i} ; 1, \boldsymbol{\beta}\right)=2 \sinh \left(\frac{y_{i}-f\left(\boldsymbol{\beta} ; \mathbf{x}_{i}\right)}{2}\right)$ and $\widehat{\tau}_{h_{u_{i}}}^{(k)}=(1 / n) \sum_{i=1}^{n}{\widehat{h^{2}}}_{u_{i}}^{(k)}$;

CM-step 2. Fix $\widehat{\alpha}^{(k+1)}$ and $\widehat{\delta}^{(k+1)}$ and update $\widehat{\boldsymbol{\beta}}^{(k)}$ using

$$
\widehat{\boldsymbol{\beta}}^{(k+1)}=\underset{\beta}{\operatorname{argmax}} Q\left(\widehat{\alpha}^{(k+1)}, \boldsymbol{\beta}, \widehat{\delta}^{(k+1)} \mid \widehat{\boldsymbol{\theta}}^{(k)}\right) .
$$

This process is iterated until convergence, i.e., the distance between two successive evaluations of the actual $\log$-likelihood $\ell(\boldsymbol{\theta})$, such as

$$
\left|\ell\left(\widehat{\boldsymbol{\theta}}^{(r+1)}\right)-\ell\left(\widehat{\boldsymbol{\theta}}^{(r)}\right)\right| \text { or }\left|\ell\left(\widehat{\boldsymbol{\theta}}^{(r+1)}\right) / \ell\left(\widehat{\boldsymbol{\theta}}^{(r)}\right)-1\right|,
$$

becomes small enough to the desired level of accuracy.

Remark 4. It is of interest to mention the following points in the implementation of the EMalgorithm described above:

1) From the above algorithm, we get the $M L$ estimate of the parameter $\delta$. Then, from the invariance property of the $M L$ estimates, we have the $M L$ estimate of $\lambda$ as $\widehat{\lambda}=\widehat{\delta} / \sqrt{1-\widehat{\delta}^{2}}$;

2) The estimates in CM-Step 1 are similar to the estimates in Santana et al. (2011). To ensure the effectiveness of the EM algorithm, suitable starting values are required. This can be done, for example, by using modified moment (MM) estimates as starting values; see Kundu et al. (2013);

3) The explicit closed-form expressions for these estimates in CM-Step 1 make the EM algorithm to be an efficient method for computing the ML estimates.

\subsubsection{The observed information matrix}

In this section, we obtain the observed information matrix of the sinh-SNI-RM, defined by

$$
\mathbf{J}_{o}(\boldsymbol{\theta} \mid \mathbf{y})=-\partial^{2} \ell(\boldsymbol{\theta} \mid \mathbf{y}) / \partial \boldsymbol{\theta} \partial \boldsymbol{\theta}^{\top}
$$


It is well known that, under some regularity conditions, the covariance matrix of the maximum likelihood estimates $\widehat{\boldsymbol{\theta}}$ can be approximated by the inverse of $\mathbf{J}_{o}(\boldsymbol{\Theta} \mid \mathbf{y})$. Following Basford et al. (1997) and Lin et al. (2007), we evaluate

$$
\mathbf{J}_{o}(\widehat{\boldsymbol{\theta}} \mid \mathbf{y})=\sum_{i=1}^{n} \widehat{\mathbf{s}}_{i}^{\top} \widehat{\mathbf{s}}_{i},
$$

where $\widehat{\mathbf{s}}_{i}=\partial\left(\log f\left(y_{i} ; \boldsymbol{\theta}_{j}\right)\right) /\left.\partial \boldsymbol{\theta}\right|_{\boldsymbol{\theta}=\widehat{\boldsymbol{\theta}}}$. We now consider the vector $\widehat{\mathbf{s}}_{i}$, which is partitioned into components corresponding to all the parameters in $\boldsymbol{\theta}$, as $\widehat{\mathbf{s}}_{i}=\left(\widehat{s}_{i, \alpha}, \widehat{s}_{i, \boldsymbol{\beta}}, \widehat{s}_{i, \lambda}\right)^{\top}$, whose components are given by

$$
\widehat{s}_{i, \alpha}=\frac{D_{\alpha}\left(f\left(y_{i} ; \boldsymbol{\theta}\right)\right)}{f\left(y_{i} ; \boldsymbol{\theta}\right)}, \quad \widehat{s}_{i, \boldsymbol{\beta}}=\frac{D_{\boldsymbol{\beta}}\left(f\left(y_{i} ; \boldsymbol{\theta}\right)\right)}{f\left(y_{i} ; \boldsymbol{\theta}\right)}, \quad \widehat{s}_{i, \lambda}=\frac{D_{\lambda}\left(f\left(y_{i} ; \boldsymbol{\theta}\right)\right)}{f\left(y_{i} ; \boldsymbol{\theta}\right)}
$$

where $D \boldsymbol{\eta}\left(f\left(y_{i} ; \boldsymbol{\theta}\right)\right)=\partial f\left(y_{i} ; \boldsymbol{\theta}\right) / \partial \boldsymbol{\eta}, \boldsymbol{\eta}=\alpha, \boldsymbol{\beta}, \lambda$. For the sinh-SN, we obtain

$$
\begin{aligned}
D_{\boldsymbol{\tau}}\left(f\left(y_{i} ; \boldsymbol{\theta}\right)\right)= & \phi\left(\xi_{2 i}\right)\left[\frac{\partial \xi_{1 i}}{\partial \boldsymbol{\tau}} \Phi\left(\lambda \xi_{2 i}\right)-\frac{1}{2} \xi_{1 i} \frac{\partial \xi_{2 i}^{2}}{\partial \boldsymbol{\eta}} \Phi\left(\lambda \xi_{2 i}\right)+\lambda \xi_{2 i} \frac{\partial \xi_{2 i}}{\partial \boldsymbol{\tau}} \phi\left(\lambda \xi_{2 i}\right)\right], \\
\boldsymbol{\tau}=\alpha \quad \text { or } \quad \boldsymbol{\beta} & \\
D_{\lambda}\left(f\left(y_{i} ; \boldsymbol{\theta}\right)\right)= & \xi_{1 i} \xi_{2 i} \phi\left(\xi_{2 i}\right) \phi\left(\lambda \xi_{2 i}\right) .
\end{aligned}
$$

Let us use the following notations

$$
\begin{aligned}
& I_{i}^{\Phi}(w)=\int_{0}^{\infty} u^{w} \exp \left\{-\frac{1}{2} u \xi_{2 i}^{2}\right\} \Phi\left(u^{1 / 2} \lambda \xi_{2 i}\right) d G(u), \\
& I_{i}^{\phi}(w)=\int_{0}^{\infty} u^{w} \exp \left\{-\frac{1}{2} u \xi_{2 i}^{2}\right\} \phi\left(u^{1 / 2} \lambda \xi_{2 i}\right) d G(u),
\end{aligned}
$$

where $i=1, \ldots, n$, for simplifying the expressions. Thus, we obtain

$$
\begin{aligned}
D_{\boldsymbol{\tau}}\left(f\left(y_{i} ; \boldsymbol{\theta}\right)\right)= & \frac{1}{\sqrt{2 \pi}}\left[\frac{\partial \xi_{1 i}}{\partial \boldsymbol{\tau}} I_{i}^{\Phi}(1 / 2)-\xi_{1 i} \xi_{2 i} \frac{\partial \xi_{2 i}}{\partial \boldsymbol{\tau}} I_{i}^{\Phi}(3 / 2)+\lambda \xi_{1 i} \frac{\partial \xi_{2 i}}{\partial \boldsymbol{\tau}} I_{i}^{\phi}(1)\right], \\
& \boldsymbol{\tau}=\alpha \quad \text { or } \quad \boldsymbol{\beta}, \\
D_{\lambda}\left(f\left(y_{i} ; \boldsymbol{\theta}\right)\right)= & \frac{\xi_{1 i} \xi_{2 i}}{\sqrt{2 \pi}} I_{i}^{\phi}(1) .
\end{aligned}
$$

Following Lachos et al. (2010a), we have closed-form expressions for the quantities $I_{i}^{\Phi}(w)$ and $I_{i}^{\phi}(w), i=1, \ldots, n$, for sinh-SCN, sinh-SSL and sinh-ST models as follows: 
- The sinh-SN model

$$
\begin{aligned}
I_{i}^{\Phi}(w) & =\sqrt{2 \pi} \phi\left(\xi_{2 i}\right) \Phi\left(\lambda \xi_{2 i}\right), \\
I_{i}^{\phi}(w) & =\phi\left(\left(\xi_{2 i}^{2}+\lambda^{2} \xi_{2 i}^{2}\right)^{1 / 2} ; 0,1\right) ;
\end{aligned}
$$

- The sinh-SCN model

$$
\begin{aligned}
I_{i}^{\Phi}(w) & =\sqrt{2 \pi}\left\{\nu \gamma^{w-1 / 2} \phi\left(\xi_{2 i} ; 0, \frac{1}{\gamma}\right) \Phi\left(\gamma^{1 / 2} \lambda \xi_{2 i}\right)+(1-\nu) \phi\left(\xi_{2 i}\right) \Phi\left(\lambda \xi_{2 i}\right)\right\} \\
I_{i}^{\phi}(w) & =\nu \gamma^{w-1 / 2} \phi\left(\left(\xi_{2 i}^{2}+\lambda^{2} \xi_{2 i}^{2}\right)^{1 / 2} ; 0, \frac{1}{\gamma}\right)+(1-\nu) \phi\left(\left(\xi_{2 i}^{2}+\lambda^{2} \xi_{2 i}^{2}\right)^{1 / 2} ; 0,1\right)
\end{aligned}
$$

- The sinh-SSL model

$$
\begin{aligned}
I_{i}^{\Phi}(w) & =\frac{2^{2+\nu} \Gamma(w+\nu)}{\left[\xi_{2 i}\right]^{2 w+2 \nu}} P_{1}\left(w+\nu, \xi_{2 i}^{2} / 2\right) E\left[\Phi\left(S_{i}^{1 / 2}\right) \lambda \xi_{2 i}\right] \\
I_{i}^{\phi}(w) & =\frac{\nu 2^{w+\nu} \Gamma(w+\nu)}{\sqrt{2 \pi}\left(\xi_{2 i}^{2}+\lambda^{2} \xi_{2 i}^{2}\right)^{w+\nu}} P_{1}\left(w+\nu, \frac{\xi_{2 i}^{2}+\lambda^{2} \xi_{2 i}^{2}}{2}\right),
\end{aligned}
$$

where $S_{i} \sim \operatorname{Gamma}\left(w+\nu, \xi_{2 i}^{2} / 2\right) I_{(0,1)}$

- The sinh-ST model

$$
\begin{aligned}
& I_{i r}^{\Phi}(w)=\frac{2^{w} \nu^{\nu / 2} \Gamma(w+\nu / 2)}{\Gamma(\nu / 2)\left(\nu+\xi_{2 i}^{2}\right)^{\nu / 2+w}} T\left(\frac{\lambda \xi_{2 i}}{\left(\xi_{2 i}^{2}+\nu\right)^{1 / 2}} \sqrt{2 w+\nu} ; 2 w+\nu\right), \\
& I_{i r}^{\phi}(w)=\frac{2^{w} \nu^{\nu / 2}}{\sqrt{2 \pi} \Gamma(\nu / 2)}\left(\xi_{2 i}^{2}+\lambda^{2} \xi_{2 i}^{2}+\nu\right)^{-\frac{\nu+2 w}{2}} \Gamma\left(\frac{\nu+2 w}{2}\right) .
\end{aligned}
$$

\subsection{Simulation study}

In this section, the quality of the estimation method (ECM algorithm) proposed in the preceding Section and the finite-sample performance of the estimates are evaluated using simulated data obtained by the Monte Carlo procedure for the regression model $Y_{i}=f\left(\boldsymbol{\beta} ; \mathbf{x}_{i}\right)+\varepsilon_{i}, i=1, \ldots, n$.

\subsubsection{Experiment 1: Recovery of parameters}

In this section, we use Monte Carlo simulations to evaluate the performance of the ML estimates of the parameters of the Sihn-SNI-RM obtained by using the proposed EM-algorithm. The samples 
sizes and the number of Monte Carlo replications considered are $n=50,100,200,400$ and $M=$ 1000, respectively, of the following regression models that are considered for illustrative purposes when the true values of the parameters are $\lambda=3$ and $\alpha=0.5,1$ :

$$
\mathrm{M} 1: f\left(\boldsymbol{\beta} ; \mathbf{x}_{i}\right)=\beta_{1}+\beta_{2} x_{1 i}+\beta_{3} x_{2 i} ; \quad \mathrm{M} 2: f\left(\boldsymbol{\beta} ; \mathbf{x}_{i}\right)=\beta_{1} z_{i 1}+\beta_{2} z i 2+\beta_{3} \exp \left(\beta_{4} x_{i}\right),
$$

where $\varepsilon_{i}$ follows the sinh-SNI distribution. For Model M1, the linear regression model (LM), we consider $\boldsymbol{\beta}=(1,2,3)^{\top}$. The results of the simulation study are only reported under the sinhST $(\nu=3)$ presented in Table 3.1 because the results were quite similar under other models. For Model M2, the nonlinear regression model $(\mathrm{NLM})$, we consider $\boldsymbol{\beta}=(4,5,3,1.5)^{\top}$ and the regression models under sinh-SN model, sinh-SCN S model $(\nu=0.1, \gamma=0.1)$, sinh-SSL $(\nu=3)$ and sinh-ST $(\nu=3)$ models. The corresponding results are in Tables 3.2-3.3 wherein the means of the parameter estimates, standard deviations (MC Sd) of the estimates across the Monte Carlo samples (MC Sd), the average (IM SE) values of the approximate standard deviations of the estimates obtained through the method described in Subsection 3.3.2, and the percentage of times that the confidence intervals cover the true value of the parameter (COV MC) are all presented. From Table 3.1 and Tables 3.2-3.3, we observe that under the sinh-ST model the performance of the EM-algorithm seems to be better for the linear regression model than the nonlinear regression model, and that it does not require a large sample size for the recovery of parameters. We also note that the estimation method of the standard errors provides relatively close results (IM SE and MC Sd) and the COV $\mathrm{MC}$ for the parameters is quite stable, indicating that the proposed asymptotic approximation for the variances of the ML estimates (Eq.(3.15)) is quite reliable. 
Table 3.1: Model M1: Mean and MC Sd are the respective mean estimates and standard deviations under the sinh-ST linear model, while IM SE is the average value of the approximate standard error obtained through the information-based method.

\begin{tabular}{|c|c|c|c|c|c|c|c|}
\hline$(\alpha)$ & $\mathrm{n}$ & Measure & $\widehat{\alpha}$ & $\widehat{\beta}_{1}(1)$ & $\widehat{\beta}_{2}(2)$ & $\widehat{\beta}_{3}(3)$ & $\widehat{\lambda}(3)$ \\
\hline \multirow{16}{*}{$(0.5)$} & \multirow[t]{4}{*}{50} & Mean & 0.4728 & 1.0249 & 1.9897 & 3.0099 & 3.4363 \\
\hline & & IM SE & 0.0988 & 0.1482 & 0.1878 & 0.1679 & 2.5514 \\
\hline & & MC Sd & 0.0913 & 0.1361 & 0.1769 & 0.1597 & 1.7402 \\
\hline & & $\mathrm{COV}$ & $92.3 \%$ & $95.3 \%$ & $95.4 \%$ & $94.2 \%$ & $96.9 \%$ \\
\hline & \multirow[t]{4}{*}{100} & Mean & 0.4927 & 1.0041 & 2.0046 & 3.0005 & 3.4737 \\
\hline & & IM SE & 0.0702 & 0.1004 & 0.1218 & 0.1133 & 1.5830 \\
\hline & & MC Sd & 0.0682 & 0.0965 & 0.1209 & 0.1144 & 1.4324 \\
\hline & & $\mathrm{COV}$ & $94.6 \%$ & $94.8 \%$ & $94.6 \%$ & $94.4 \%$ & $95.4 \%$ \\
\hline & \multirow[t]{4}{*}{200} & Mean & 0.4971 & 1.0056 & 1.9991 & 2.9963 & 3.2637 \\
\hline & & IM SE & 0.0499 & 0.0682 & 0.0834 & 0.0799 & 0.9773 \\
\hline & & MC Sd & 0.0495 & 0.0690 & 0.0810 & 0.0816 & 1.0487 \\
\hline & & $\mathrm{COV}$ & $94.9 \%$ & $94.6 \%$ & $95.5 \%$ & $94.2 \%$ & $95.3 \%$ \\
\hline & \multirow[t]{4}{*}{400} & Mean & 0.4975 & 1.0012 & 1.9998 & 3.0035 & 3.0859 \\
\hline & & IM SE & 0.0354 & 0.0502 & 0.0607 & 0.0590 & 0.6263 \\
\hline & & MC Sd & 0.0345 & 0.0489 & 0.0616 & 0.0604 & 0.6051 \\
\hline & & $\mathrm{COV}$ & $95.0 \%$ & $95.5 \%$ & $94.9 \%$ & $94.1 \%$ & $96.3 \%$ \\
\hline \multirow{16}{*}{$(1.0)$} & \multirow[t]{4}{*}{50} & Mean & 0.9443 & 1.0410 & 1.9872 & 3.0177 & 3.4661 \\
\hline & & IM SE & 0.2212 & 0.3020 & 0.3870 & 0.3599 & 2.6455 \\
\hline & & MC Sd & 0.1875 & 0.2749 & 0.3558 & 0.3398 & 1.7190 \\
\hline & & $\mathrm{COV}$ & $94.4 \%$ & $95.2 \%$ & $95.1 \%$ & $94.4 \%$ & $99.1 \%$ \\
\hline & \multirow[t]{4}{*}{100} & Mean & 0.9862 & 1.0118 & 2.0070 & 3.0065 & 3.5109 \\
\hline & & IM SE & 0.1569 & 0.2280 & 0.2568 & 0.2195 & 1.6856 \\
\hline & & MC Sd & 0.1513 & 0.2275 & 0.2450 & 0.2252 & 1.5215 \\
\hline & & $\mathrm{COV}$ & $94.2 \%$ & $94.5 \%$ & $95.5 \%$ & $93.6 \%$ & $97.2 \%$ \\
\hline & \multirow[t]{4}{*}{200} & Mean & 0.9929 & 1.0035 & 1.9968 & 3.0071 & 3.2769 \\
\hline & & IM SE & 0.1112 & 0.1477 & 0.1600 & 0.1648 & 1.0511 \\
\hline & & $\mathrm{MC} \mathrm{Sd}$ & 0.1094 & 0.1494 & 0.1571 & 0.1647 & 1.0667 \\
\hline & & $\mathrm{COV}$ & $94.5 \%$ & $94.5 \%$ & $95.1 \%$ & $95.1 \%$ & $95.7 \%$ \\
\hline & \multirow[t]{4}{*}{400} & Mean & 0.9932 & 1.0077 & 1.9999 & 2.9961 & 3.0976 \\
\hline & & IM SE & 0.0789 & 0.0990 & 0.1158 & 0.1152 & 0.6785 \\
\hline & & $\mathrm{MC} \mathrm{Sd}$ & 0.0769 & 0.1003 & 0.1199 & 0.1152 & 0.6781 \\
\hline & & $\mathrm{COV}$ & $95.7 \%$ & $94.9 \%$ & $94.1 \%$ & $93.7 \%$ & $96.0 \%$ \\
\hline
\end{tabular}


资

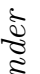

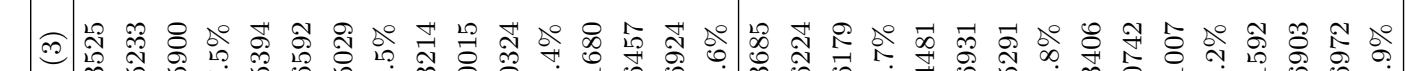
《र

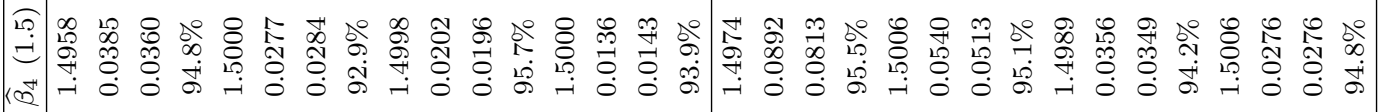

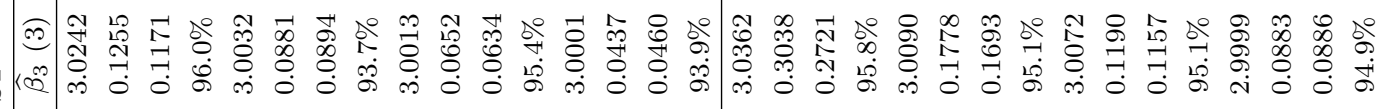

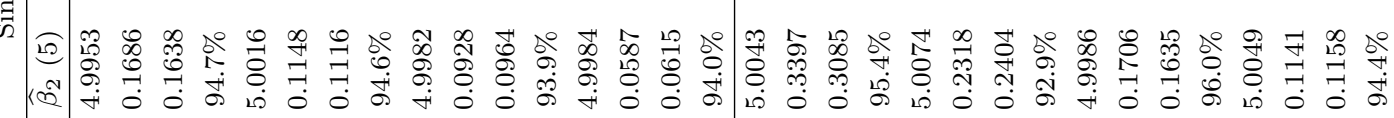

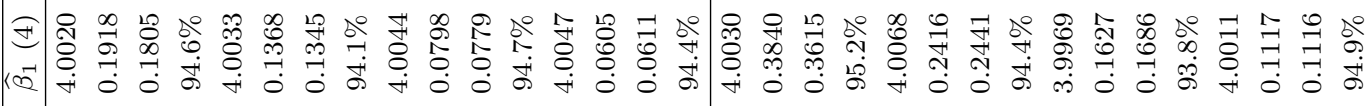

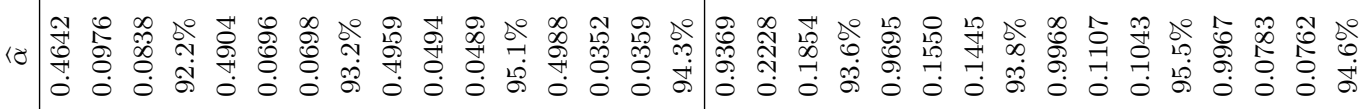

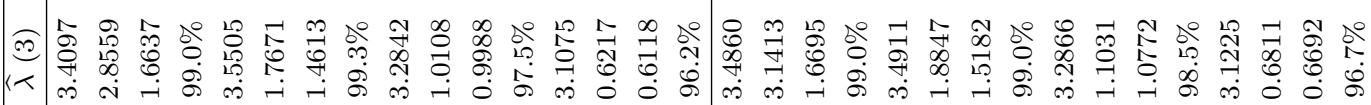

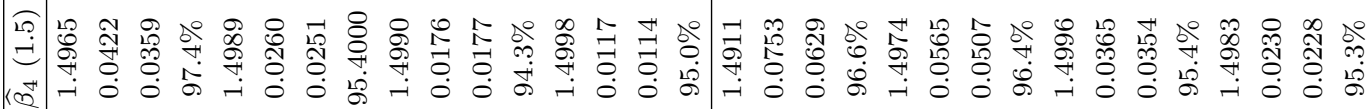
శึ. $\frac{0}{3} \stackrel{0}{\pi}$ हैं

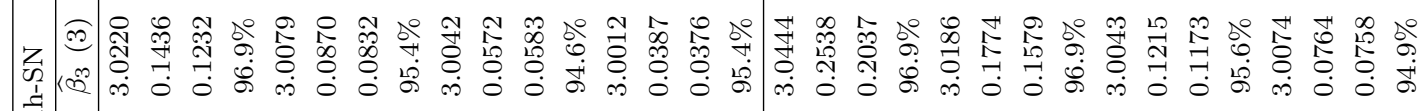
क्ष

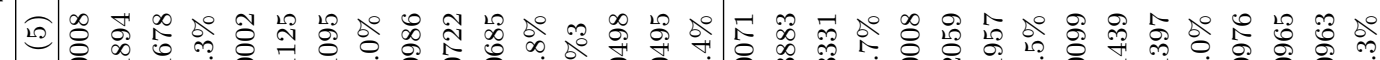
« 离离离

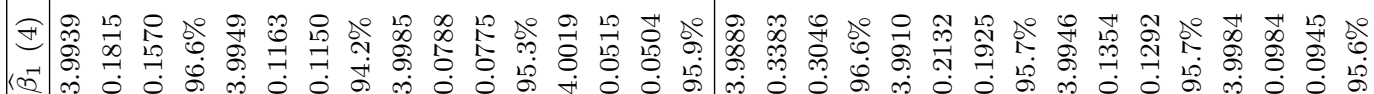
है

$\stackrel{8}{\circ}$

‡્丶 \&

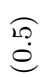

$\stackrel{\overbrace{}}{i}$ 
$\stackrel{\stackrel{\Xi}{\Xi}}{3}$

$\frac{\infty}{0}$

胥

¿

कू

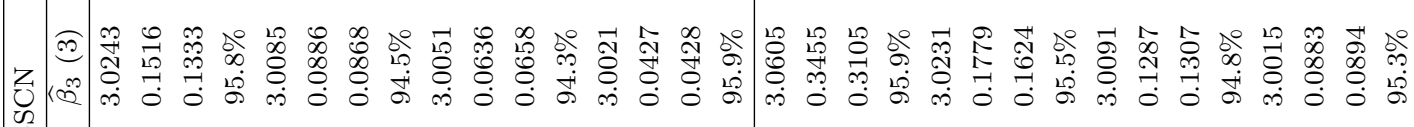

త్రిత్ర

जे

.

$\frac{0}{\pi}$

ฐึ๊

है है

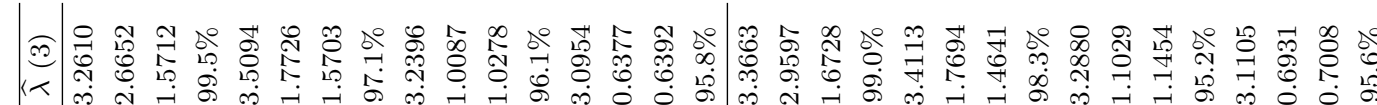

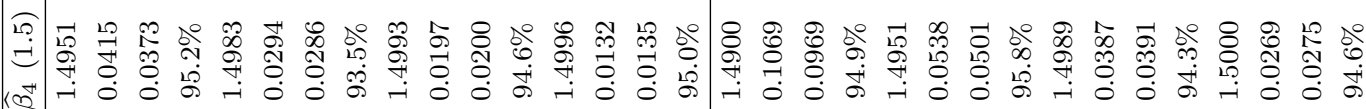
(ख)

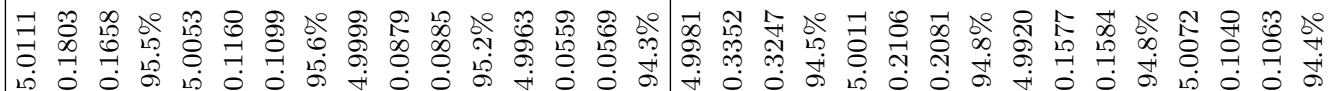

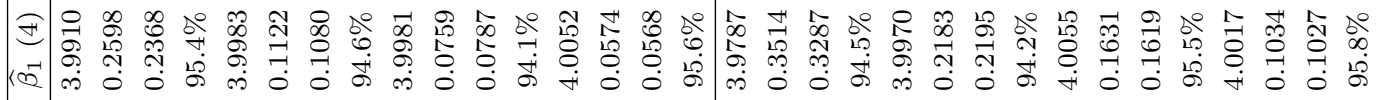

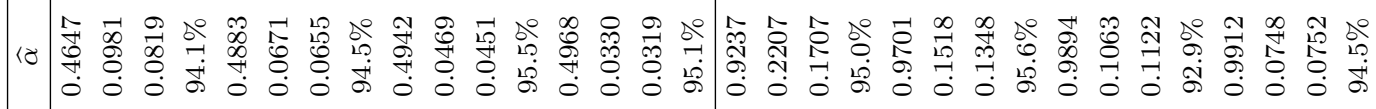

(⿻)

के

(艹)

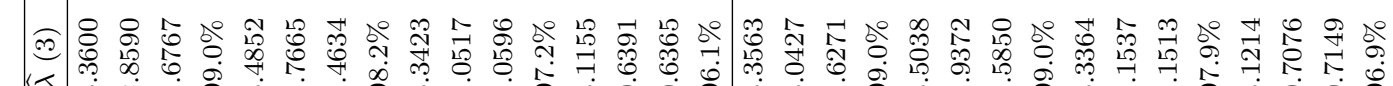

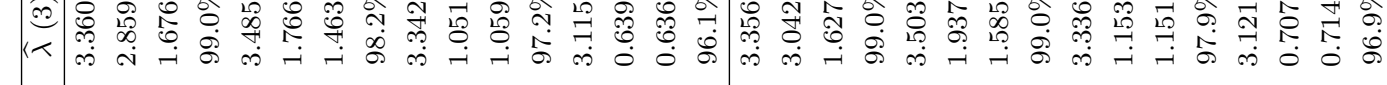

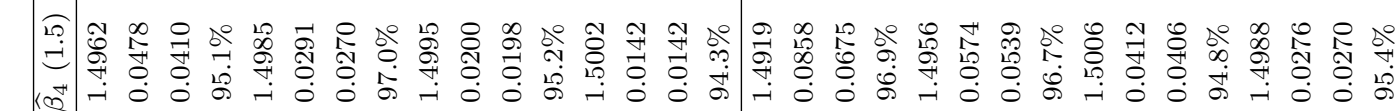

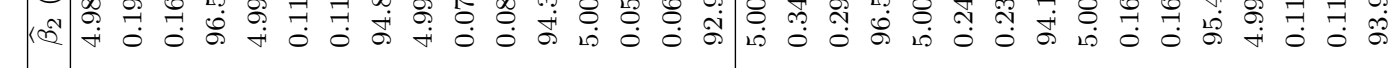

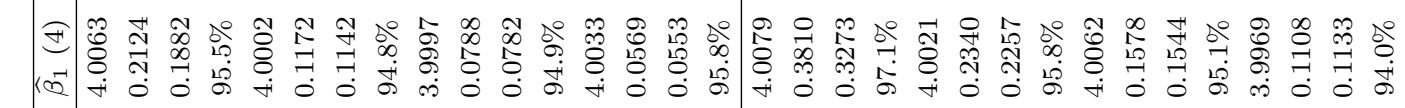




\subsubsection{Experiment 2: Asymptotic properties}

Our aim here is to demonstrate the asymptotic properties of the EM estimates. We chose sample sizes $n=100,250,500,800,1000,1500,2000,3000,4000$ and5000, from Model M2 with the choice of parameters $\alpha=0.5, \boldsymbol{\beta}=(4,5,3,1.5)^{\top}$ and $\lambda=3$. For each combination of parameters and sample size, we generated 1000 random samples from Model M2 under $\sinh -\mathrm{ST}(\nu=3)$, $\sinh -\mathrm{SSL}(\nu=3)$ and $\sinh -\mathrm{SCN}(\nu=0.1, \gamma=0.1)$ models. From the EM estimates of model parameters determined, we computed the root mean square error (RMSE), the bias (Bias) and the Monte Carlo standard deviation (MC-SD) for each parameter over the 1000 replicates. If $\theta_{k}$ is a component of $\boldsymbol{\theta}$, then these quantities are defined, respectively, by

$$
\operatorname{MC-SD}\left(\widehat{\theta}_{k}\right)=\frac{1}{m-1} \sum_{j=1}^{m}\left(\widehat{\theta}_{k}^{(j)}-\widehat{\widehat{\theta}}_{k}\right)^{2} \text { and } \operatorname{RMSE}\left(\widehat{\theta}_{k}\right)=\sqrt{\operatorname{MC}_{-\operatorname{Sd}^{2}}\left(\widehat{\theta}_{k}\right)+\operatorname{Bias}^{2}\left(\widehat{\theta}_{k}\right)}
$$

where $\operatorname{Bias}\left(\widehat{\theta}_{k}\right)=\overline{\widehat{\theta}_{k}}-\theta_{k}, \overline{\widehat{\theta}_{k}}=\sum_{j=1}^{m} \widehat{\theta}_{k}^{(j)} / m$ is the Monte Carlo mean and $\theta_{k}^{(j)}$ is the estimate of $\theta_{k}$ from the $j$ th sample, with $j=1, \ldots, m$. Figures 3.3 and 3.4 present the Bias and RMSE of the ML estimates of the parameters $\alpha, \boldsymbol{\beta}$, and $\lambda$. It is observed that the Bias and RMSE become smaller as the sample size $n$ increases, as one would expect. We also observe that the Bias of the ML estimate of $\lambda$ is relatively larger than that of the ML estimates of $\alpha$ and $\boldsymbol{\beta}$.

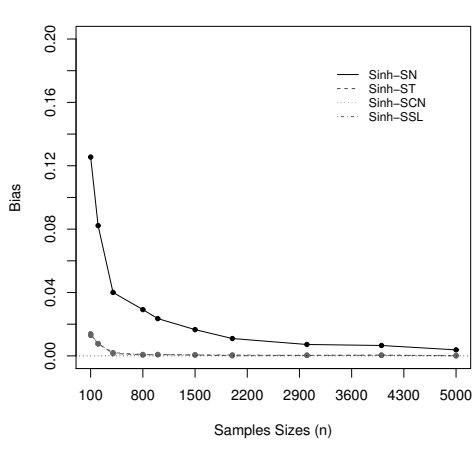

$\beta_{3}$

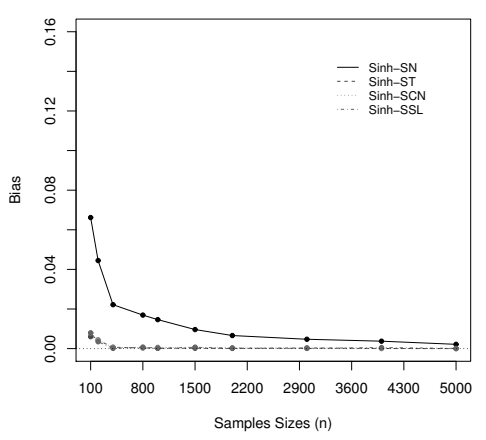

$\beta_{1}$

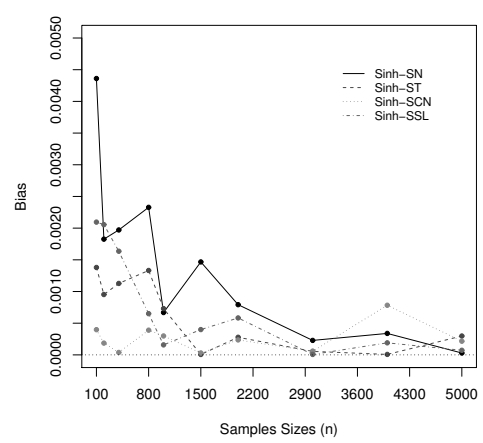

$\beta_{4}$

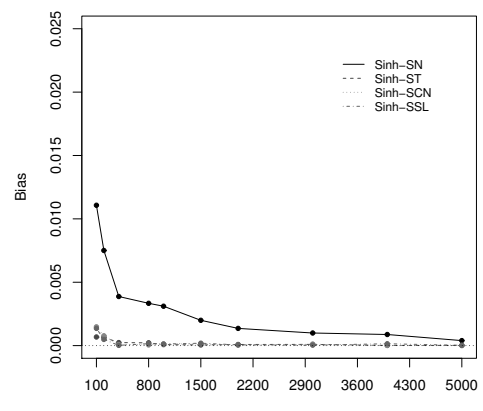

Samples Sizes (n)

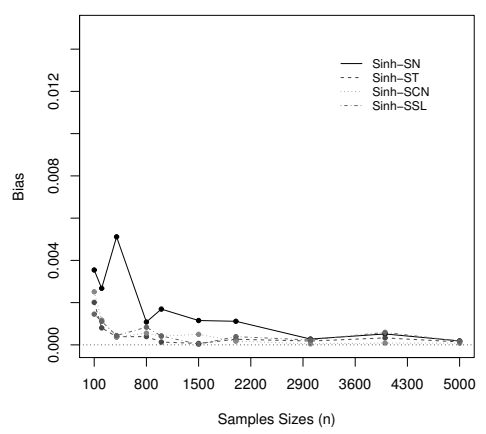

$\lambda$

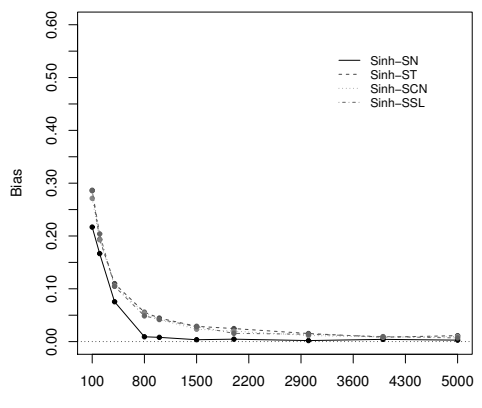

Figure 3.3: Bias of parameters $\alpha, \beta_{1}, \beta_{2}, \beta_{3}, \beta_{4}$, and $\lambda$ under sinh-SNI model. 
$\alpha$

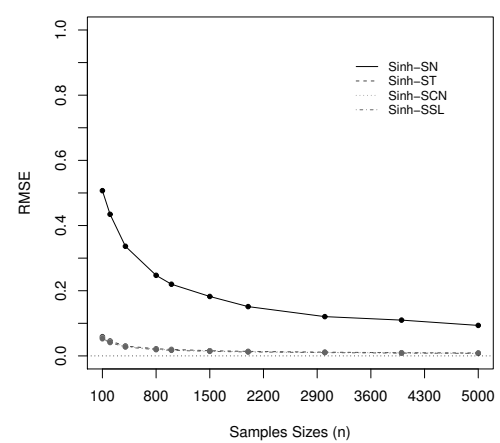

$\beta_{3}$

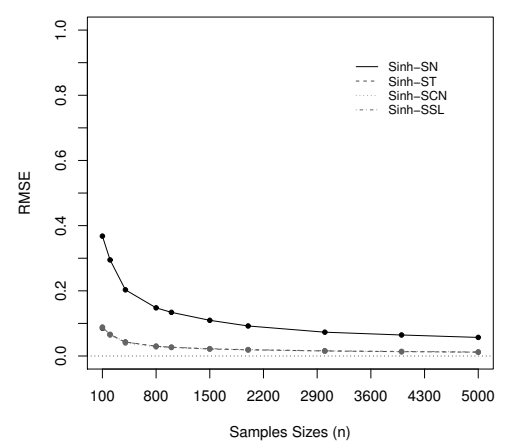

$\beta_{1}$

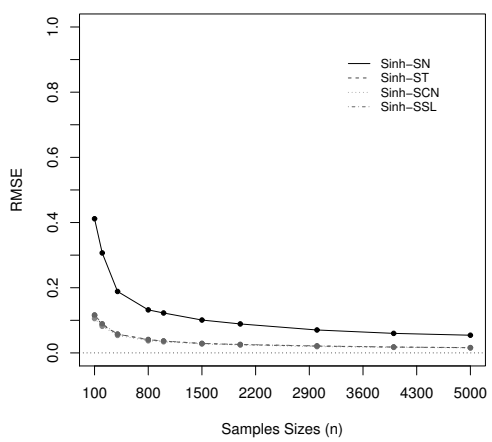

$\beta_{4}$

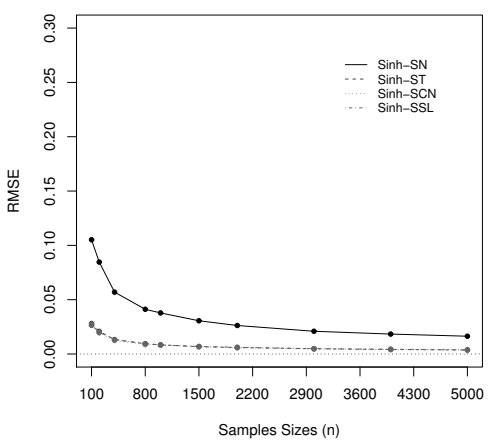

$\beta_{2}$
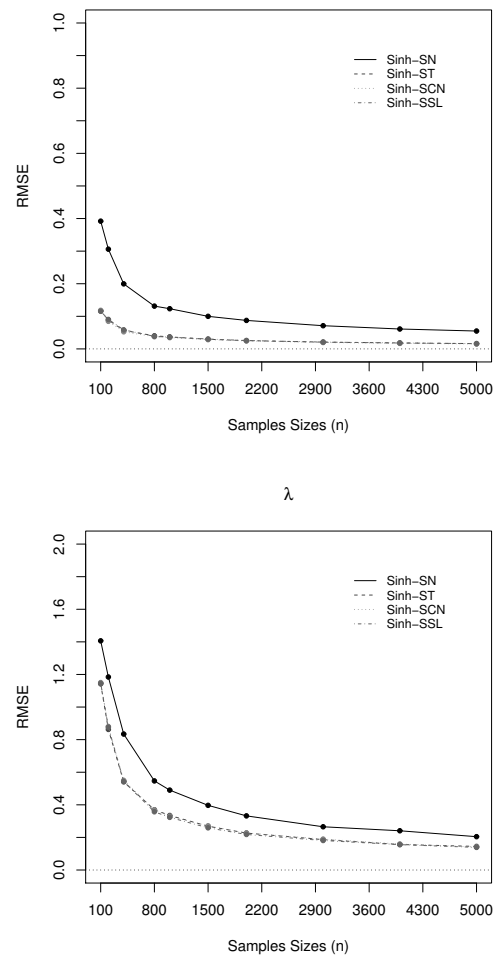

Figure 3.4: RMSE of MLEs of parameters $\alpha, \beta_{1}, \beta_{2}, \beta_{3}, \beta_{4}$, and $\lambda$ under sinh-SNI model.

\subsection{Illustrative examples}

In this section, we fit the proposed models to two datasets analized earlier by Rieck and Nedelman (1991) and Lin (1994). These examples are used to illustrate the flexibility of the proposed linear and nonlinear regression models under sinh-SN, sinh-ST sinh-SCN and sinh-SSL models. Standard errors (SE) are obtained by the method outlined in Section 3.3.2. The goodness-of-fit is evaluated through

$$
d_{i}=d\left(Y_{i}, \widehat{\boldsymbol{\theta}}\right)=\xi_{2}^{2}\left(Y_{i} ; \widehat{\alpha}, \widehat{\mu}_{i}, 2\right)
$$

mentioned earlier in Part (iii) of Remark 5, where $\widehat{\boldsymbol{\theta}}$ is the ML estimate of $\boldsymbol{\theta}$. For model selection criteria, we computed the log-likelihood values $\ell(\boldsymbol{\theta})$, the Akaike information criterion (AIC), the Bayesian information criterion (BIC), and the efficient determination criterion (EDC).

\subsubsection{Biaxial fatigue dataset}

This dataset was first by analyzed by Rieck and Nedelman (1991) in linear regression model. These data were considered by Farias and Lemonte (2011) in a nonlinear regression model and Santana et al. (2011) in a BS linear regression model by using the skew-normal distribution. These data correspond to 46 metal pieces that were tested until failure. The variables considered in this 
study are the number of cycles until failure $(N)$ and the work per cycle (in MJ / $\mathrm{m}^{3}$ ). Lemonte and Cordeiro (2009) proposed the nonlinear regression model

$$
Y_{i}=\beta_{1}+\beta_{2} \exp \left(-\beta_{3} / x_{i}\right)+\varepsilon_{i}, \quad i=1, \ldots, 46,
$$

where $Y_{i}=\log \left(N_{i}\right)$ is the logarithm of the number of cycles, $x_{i}$ is the $i$ th value of the work per cycle, $\beta_{1}, \beta_{2}$ and $\beta_{3}$ the regression coefficients, and $\varepsilon_{i} \sim \operatorname{Sinh}-\mathrm{N}(\alpha, 0, \sigma=2)$. Here, we consider $\varepsilon_{i} \sim \operatorname{Sinh}-\operatorname{SNI}(\alpha, 0, \sigma=2 ; H)$, based on SN-NLM, ST-NLM, SCN-NLM and SSL-NLM. Table 3.4 provides the ML estimates of the model parameters along with their standard errors (SE). Moreover, the log-likelihood function at the ML estimates and the information selection criteria such as AIC, BIC and EDC criteria (sinh-NI-NLM, in parentheses) are all reported. The criteria values indicate that the sinh-SNI-NLM models with heavy tails provide a significantly better fit than the sinh-NI-NLM model. Next, upon substituting the ML estimate of $\boldsymbol{\theta}$ in $d\left(Y_{i}\right)$, the goodnessof-fit presented in Figure 3.5 displays simulated envelopes (lines representing the 5th percentile, the mean and the 95 th percentile of 200 simulated points for each observation). This figure provides strong evidence that the sinh-SNI-NLM provides a better fit to these data than the sinh-NI-NLM model, with the best model being the SN-NLM.

Table 3.4: Biaxial dataset: ML estimates for the four selected sinh-SNI-NLMs, the log-likelihood values and the information criteria values, with sinh-NI-NLMs (in parentheses).

\begin{tabular}{c|cc|cc|cc|cc}
\hline \hline \multirow{2}{*}{ Parameter } & \multicolumn{2}{|c|}{ Sinh-SN } & \multicolumn{2}{c|}{ Sinh-ST } & \multicolumn{2}{c|}{ Sinh-SCN } & \multicolumn{2}{c}{ Sinh-SSL } \\
& Estimate & SE & Estimate & SE & Estimate & SE & Estimate & SE \\
\hline$\alpha$ & 0.7026 & 0.1185 & 0.6673 & 0.1223 & 0.6110 & 0.1080 & 0.67350 & 0.11419 \\
$\beta_{1}$ & 10.0779 & 1.0045 & 10.1018 & 0.9945 & 10.0910 & 0.9978 & 10.07918 & 1.00373 \\
$\beta_{2}$ & -5.6500 & 0.6608 & -5.6817 & 0.6667 & -5.6675 & 0.6632 & -5.65171 & 0.66098 \\
$\beta_{3}$ & -18.8645 & 7.3732 & -18.7276 & 7.1313 & -18.7920 & 7.2333 & -18.85769 & 7.35884 \\
$\lambda$ & -6.9827 & 8.3388 & -6.4896 & 7.4741 & -6.7127 & 7.8613 & -6.95720 & 8.29373 \\
$\nu$ & - & - & 16.4 & - & 0.5 & - & 12.1 & - \\
$\gamma$ & - & - & - & - & 0.6 & - & - & - \\
\hline$l(\widehat{\boldsymbol{\theta}})$ & $-19.3556(-22.3862)$ & $-19.8041(-22.8317)$ & $-19.5798(-22.5373)$ & $-19.3811(-22.2623)$ \\
AIC & $48.7112(52.7724)$ & $49.6082(53.6635)$ & $49.1596(53.0746)$ & $48.7622(52.5246)$ \\
BIC & $57.8544(60.0869)$ & $58.7515(60.9781)$ & $58.3028(60.3891)$ & $57.9054(59.8391)$ \\
EDC & $45.4935(50.1982)$ & $46.3906(51.0894)$ & $45.9419(50.5004)$ & $45.5445(49.9504)$ \\
\hline \hline
\end{tabular}

Figure 3.6 displays the scatter plots of the dataset and the fitted nonlinear models based on SNI and NI distributions. From this figure, we once again see strong evidence for the sinh-SNI-NLM model providing a better fit to these data. 

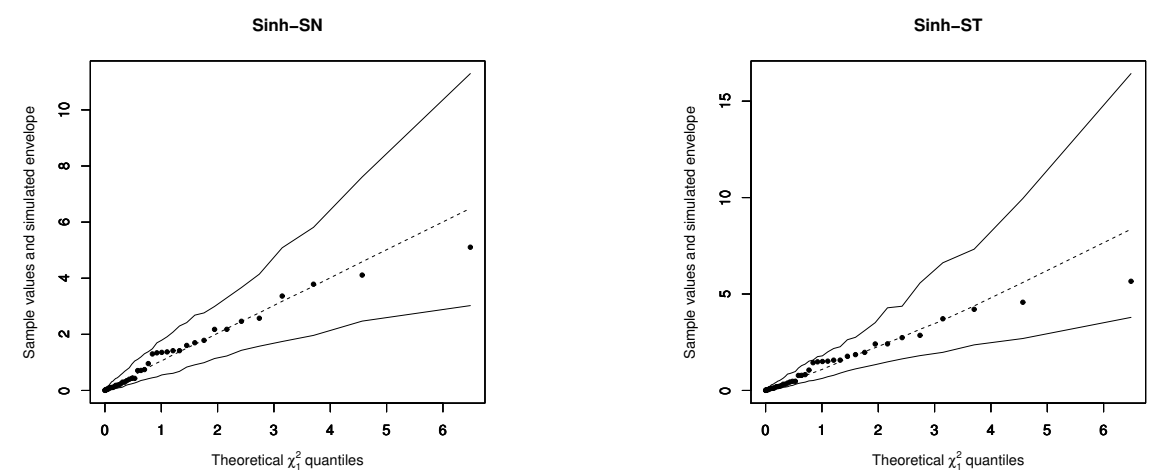

Sinh-SCN

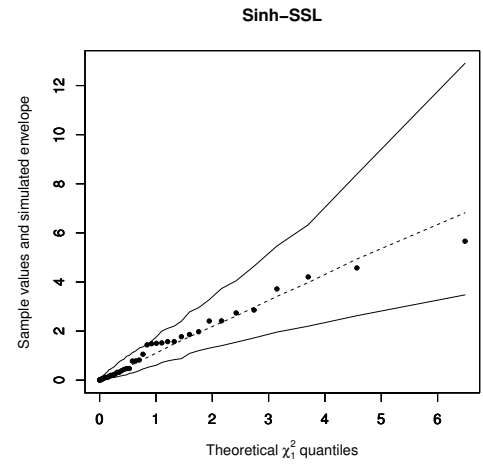

Figure 3.5: Biaxial dataset: Simulated envelopes for sinh-SNI-NLM based on SN, ST, SCN and SSL distributions.

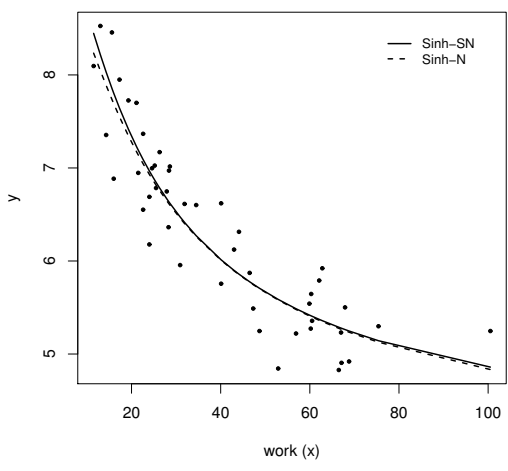

(a)

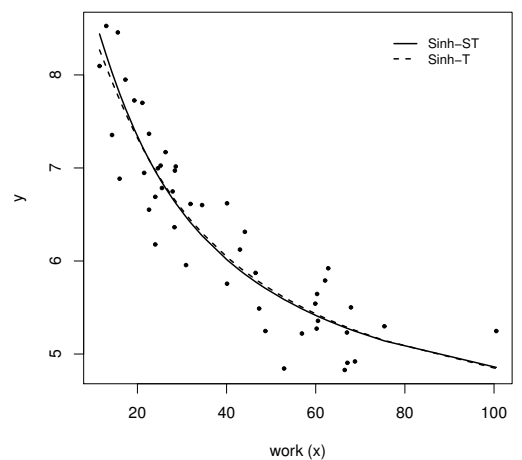

(b)

Figure 3.6: Biaxial dataset: Scatterplots and fitted lines based on sinh-SN-NLM and sinh-ST-NLM models.

\subsubsection{Martin Marietta dataset}

Here, we consider the Martin Marietta dataset analyzed by Ferreira et al. (2015) and Butler et al. (1990). This data set was also studied by Azzalini and Capitanio (2003) under asymmetrical 
models. On the basis of arguments presented by them, we consider here the linear regression

$$
Y_{i}=\beta_{0}+\beta_{1} \mathrm{CRSP}_{i} \varepsilon_{i}, \quad i=1, \ldots, 60,
$$

where the response $Y$ is the excess rate of return of the Martin Marietta company and CRSP is an index of the excess rate of return for the New York market as a whole. We assume that the error terms are distributed as $\varepsilon_{i} \sim \sinh -\operatorname{SNI}(\alpha, 0, \sigma=2 ; H)$. Data over a period of $n=60$ consecutive months are available. Table 3.5 displays the ML estimates of the model parameters under the sinh-SN, sinh-ST, sinh-SCN and sinh-SSL models and their corresponding standard errors (SE). For model comparison, we computed the $\ell(\boldsymbol{\theta})$ values, and the AIC, BIC and EDC criteria (sinh-NI model, in parentheses) values. The criteria values reveal that the regression model under sinh-SNI models with heavy tails present a significantly better fit than the symmetric sinh-NI models. We also note that the fit under the sinh-SCN and sinh-SSL models are significantly better than the sinh-SN model.

Table 3.5: Martin Marietta dataset: Results from fitting four selected sinh-SNI models (information criteria values under sinh-NI model, in parentheses).

\begin{tabular}{c|cc|cc|cc|cc}
\hline \hline \multirow{2}{*}{ Parameter } & \multicolumn{2}{|c|}{ Sinh-SN } & \multicolumn{2}{c|}{ Sinh-ST } & \multicolumn{2}{c|}{ Sinh-SCN } & \multicolumn{2}{c}{ Sinh-SSL } \\
& Estimate & SE & Estimate & SE & Estimate & SE & Estimate & SE \\
\hline$\alpha$ & 0.1373 & 0.0132 & 0.0625 & 0.0138 & 0.0670 & 0.0178 & 0.0493 & 0.0121 \\
$\beta_{0}$ & -0.0937 & 0.0143 & -0.0472 & 0.0210 & -0.0484 & 0.0264 & -0.0476 & 0.0237 \\
$\beta_{1}$ & 1.3891 & 0.3839 & 1.2455 & 0.2138 & 1.2665 & 0.2184 & 1.2631 & 0.2182 \\
$\lambda$ & 3.9496 & 2.0794 & 1.0841 & 0.8518 & 1.0594 & 0.9849 & 1.0717 & 0.9193 \\
$\nu$ & - & - & 3.1819 & - & 0.0836 & - & 1.1903 & - \\
$\gamma$ & - & - & - & - & 0.0526 & - & - & - \\
$\ell(\widehat{\boldsymbol{\theta}})$ & $65.7676(57.2889)$ & $73.0699(71.8111)$ & $74.0996(72.9468)$ & $73.6296(72.4625)$ \\
AIC & $-123.5353(-108.5779)$ & $-138.1398(-137.6221)$ & $-140.1992(-139.8936)$ & $-139.2592(-138.925)$ \\
BIC & $-115.1579(-102.2948)$ & $-129.7625(-131.3391)$ & $-131.8218(-133.6106)$ & $-130.8818(-132.642)$ \\
EDC & $-125.3385(-109.9303)$ & $-139.9431(-138.9746)$ & $-142.0024(-141.2461)$ & $-141.0624(-140.2775)$ \\
\hline \hline
\end{tabular}

Figure 3.7 displays the scatter plots of the dataset and the fitted linear models based on the sinh-SN and sinh-SCN models. These fitted linear models are similar to those obtained by Ferreira et al. (2015). From this figure, we can note some possible outlier observations, such as \#8, \#15 and \#34, under the fitted models. However, the effect of these observations are attenuated through the use of the sinh-SNI distributions as we will see later on.

In addition, Figure 3.8 displays simulated envelopes (lines representing the 5th percentile, the mean and the 95 th percentile of 200 simulated points for each observation) based on $d_{i}$ values 

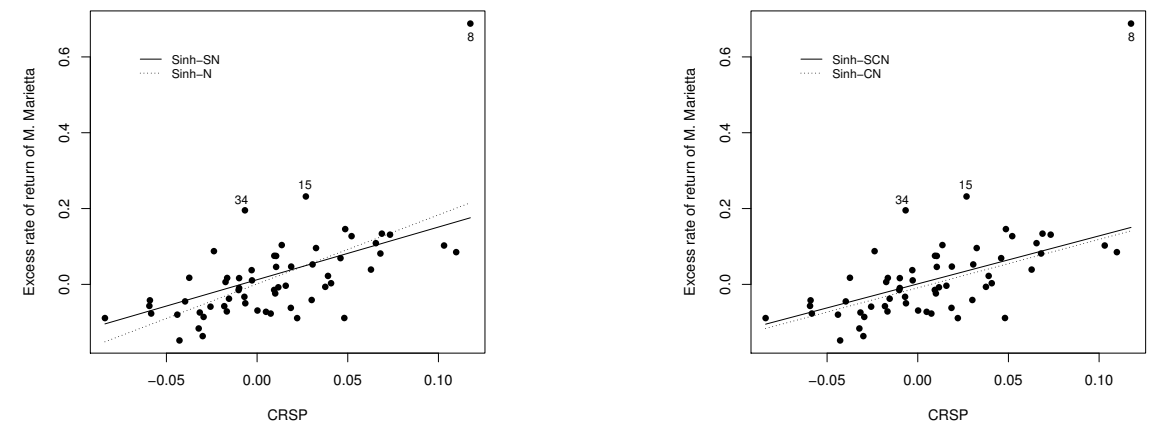

Figure 3.7: Martin Marietta dataset: Scatterplots and fitted lines under the sinh-SN (left) and sinh-SCN (right) models.

which once again strongly supports that the models with heavy tails produce a better fit to the current data, with the sinh-SCN and sinh-SSL models being significantly better.
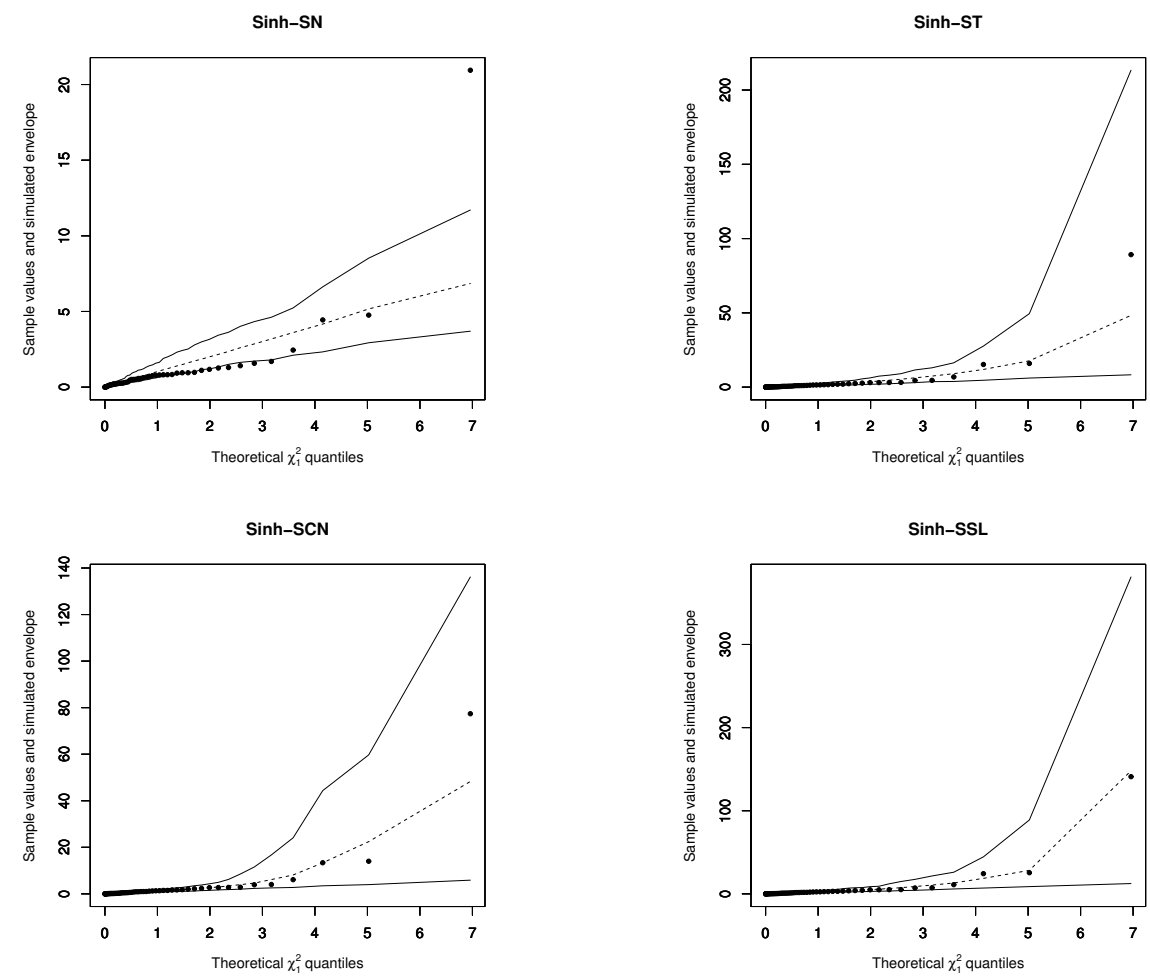

Figure 3.8: Martin Marietta dataset: Simulated envelopes under the four proposed models.

To identify outlying observations, we use the Mahalanobis distance $d_{i}$. Figure 3.9 displays such distances for the four fitted models, where the cutoff lines correspond to the quantile $\tau=0.95$. From the figure, we note that the observations \#8, \#15 and \#34 are detected as possible outliers under the fitted models. From the EM-algorithm, the estimated weights $\left(\widehat{\kappa}_{i}, i=1, \ldots, 60\right)$ for these observations are the smallest ones for all fitted asymmetric models with heavier tails as can be seen in Figure 3.10, thus confirming the robustness aspects of the ML estimates against outlying 
observations of the heavy-tailed sinh-SNI models, where segmented lines correspond to the sinhnormal model in which case $\kappa_{i}=1$, for all $i$. Here, we note that larger $d_{i}$ values imply a smaller $\kappa_{i}$ values, and so in the process of estimation of $\boldsymbol{\theta}$, little weight is given to the outliers. To reveal the impact of the detected influential observations, we follow the suggestion of Lee et al. (2006) and use the quantities TRC and MRC defined as follows:

$$
\mathrm{TRC}=\sum_{j=1}^{n_{p}}\left|\frac{\widehat{\theta}_{j}-\widehat{\theta}_{[i] j}}{\widehat{\theta}_{j}}\right| \quad \text { and } \quad \mathrm{MRC}=\max _{j=1, \ldots, n_{p}}\left|\frac{\widehat{\theta}_{j}-\widehat{\theta}_{[i] j}}{\widehat{\theta}_{j}}\right| \text {, }
$$

where $n_{p}$ is the dimension of $\boldsymbol{\theta}$ and the subscript [i] stands for the ML estimate of $\boldsymbol{\theta}$ when the $i$ th observation is deleted. Table 3.6 shows the comparisons of these measures for the fitted models without the observation \#8 where the biggest change takes place under the Sinh-SN distribution. As expected, the results indicate that the ML estimates are less sensitive in the presence of atypical data when distributions with heavier tails are assumed than the sinh-SN model.
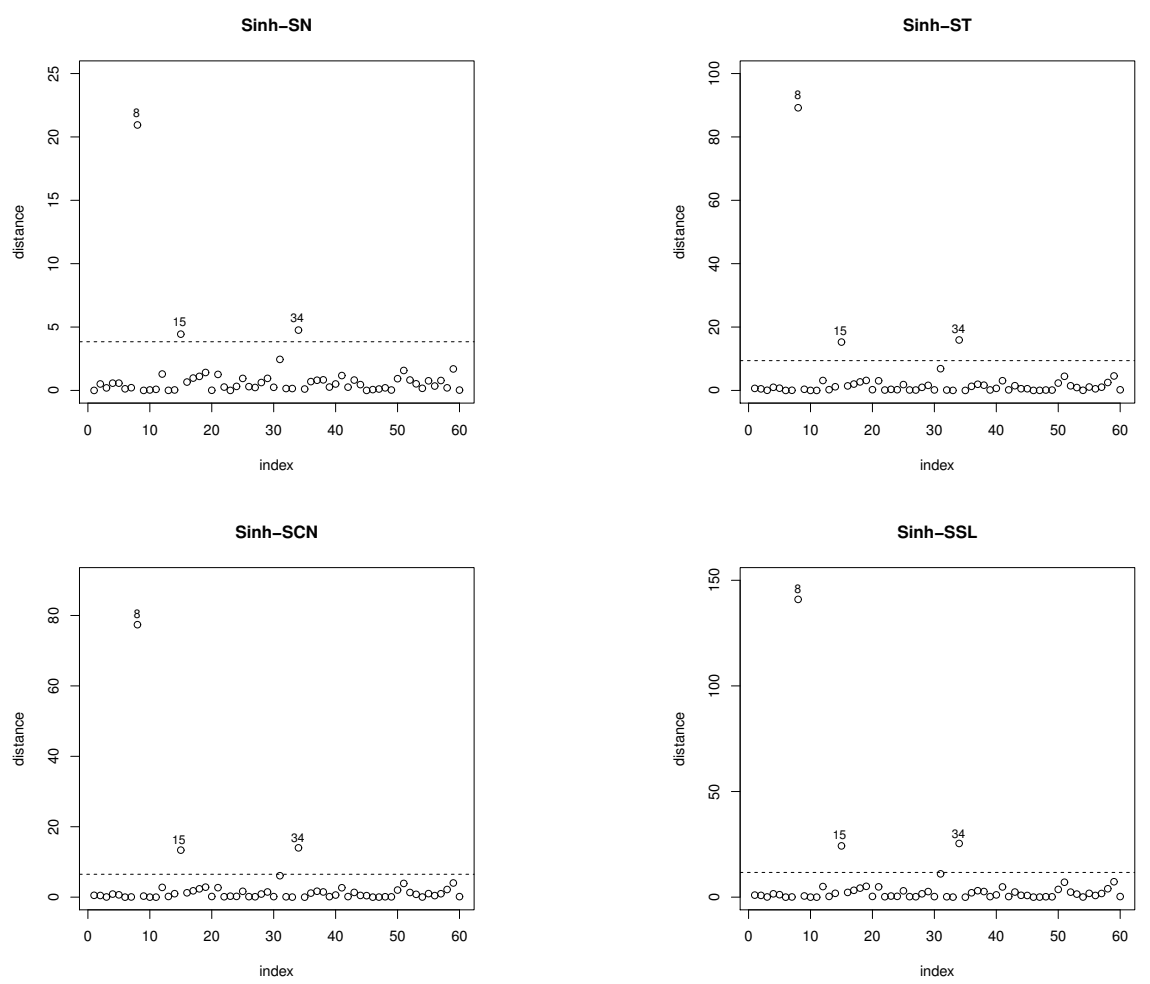

Figure 3.9: Martin Marietta dataset: Index plots of the distances $\left(d_{i}\right)$ for the fitted models. 

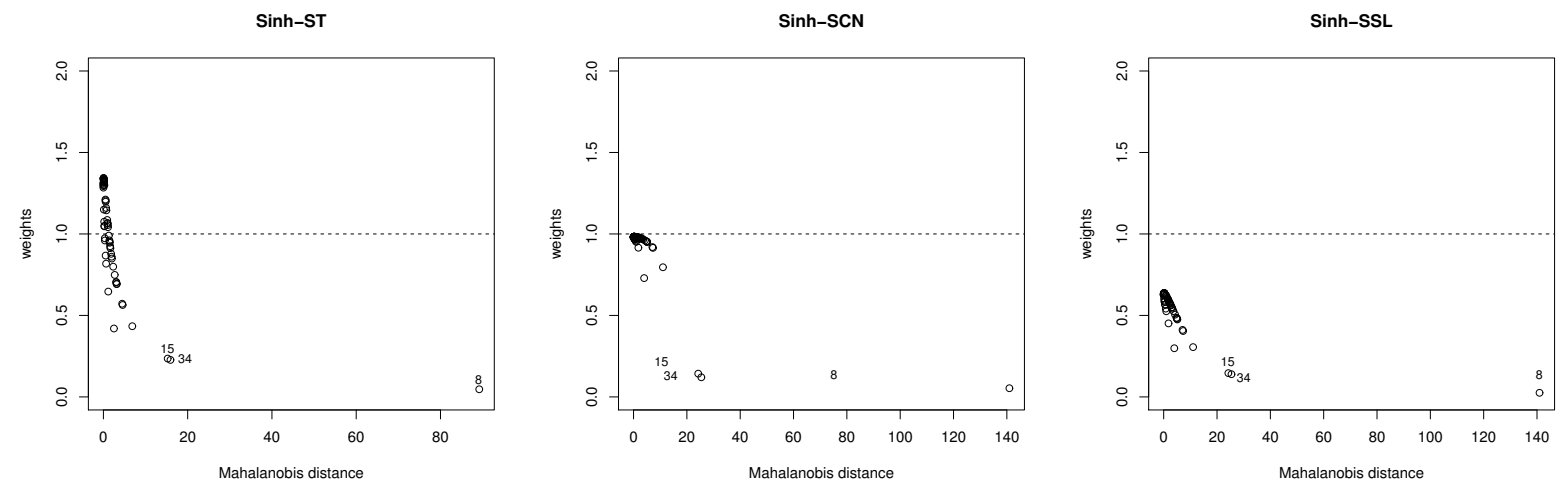

Figure 3.10: Martin Marietta dataset: Estimated $\kappa_{i}$ for the ST, SCN and SSL models.

Table 3.6: Martin Marietta dataset: Comparison of the relative changes in the ML estimates in term of TRC and MRC for the four selected sihn-SNI models.

\begin{tabular}{ccc}
\hline \hline & TRC & MRC \\
\hline Sinh-SN & 11.6174 & 9.8691 \\
Sinh-ST & 2.3682 & 1.2204 \\
Sinh-SCN & 1.9838 & 0.9829 \\
Sinh-SSL & 2.3232 & 1.2041 \\
\hline \hline
\end{tabular}

\subsection{Concluding remarks}

Nonlinear regression analysis is a very useful methodology that has been used extensively in several fields, and this paper provides useful results that can be used in survival and reliability studies when asymmetric distributions are assumed. Specifically, we first introduce a general class of generalized sinh-Normal distributions, called sinh-SNI distributions, that is based on the skewnormal/Independent distribution instead of the normal distribution. So, this proposal generalizes some results presented earlier by Rieck and Nedelman (1991), Balakrishnan et al. (2009) and Vilca et al. (2015), among others. Some special cases of this general model are the asymmetric sinh-SN, sinh-ST, sinh-SCN and sinh-SSL distributions, and an important case is the log-Birnbaum-Saunders distribution that has been used in the linear regression model setup. Second, we propose Sinh-SNI nonlinear regression models which generalize linear and nonlinear regression models that have been developed so far by considering symmetric generalized sinh-normal distributions developed by some authors including Rieck and Nedelman (1991), Lemonte and Cordeiro (2009), Paula et al. (2011) and Vilca et al. (2015). Furthermore, we have presented an EM-algorithm for the ML estimation 
of model parameters, in which the CM-Step provides closed-form expressions for the estimates of $\alpha$ and $\lambda$ that allow us to obtain qualitatively robust ML estimates in an efficient manner, as seen in the numerical illustrations. This generalization results in making the inference robust to the presence of outlying observations, as demonstrated in Section 3.5, and is quite a flexible and useful distribution for modeling purposes. 


\title{
Chapter 4
}

\section{Influence analysis in}

\section{Sinh-skew-normal/Independent}

\section{regression}

\section{models}

\begin{abstract}
Nonlinear regression models are important tools since many processes are better represented by nonlinear relationship than linearrelationship. Here we propose methods to detect influential observations in sinh-skew-normal/independent regression models, which contain as special cases the well-known Birnbaum-Saunders regression models (Rieck and Nedelman, 1991), which are useful in lifetime data analysis. We propose methods to detect influential observations in such models, on the basis of maximum likelihood estimates obtained through an EM algorithm. Specifically, we develop the diagnostic measures on the basis of the conditional expectation of the completedata log-likelihood function in the EM algorithm, because the observed-data log-likelihood function associated with the proposed model is somewhat complex, making it difficult to directly apply the approach of Cook $(1977,1986)$. Diagnostic measures are derived from the case-deletion approach and the local influence approach. Finally, a simulation study and two real datasets are used to illustrate the usefulness of the proposed method.
\end{abstract}


Keywords: Influence analysis; case-deletion; local influence; sinh-normal distribution; BirnbaumSaunders distribution; skew-normal distribution; scale mixtures of skew-normal distribution.

\subsection{Introduction}

The nonlinear regression models are applied in different areas of investigation, such as science and engineering. Several of these models have found applications for analysis of lifetime datasets based on the exponential, Weibull, lognormal and gamma distributions. The univariate sinh-normal distribution has received considerable attention in lifetime regression models, especially when the lifetime data involve modeling by the distribution of Birnbaum and Saunders (1969a,b), in which the scale parameter depends on the covariates. The Birnbaum-Saunders (BS) distribution has applications in a wide range of problems, as does the sinh-normal distribution due to its close relationship with the BS distribution (Rieck, 1989). These distributions are built by using the normal distribution. Hence, the sinh-normal distribution may not be suitable to represent data containing outlying observations due to its close dependence on the normal distribution. To overcome this problem, the relationship between the sinh-normal and normal distributions has been exploited to obtain generalizations of the general class of sinh-normal distributions based on the family of normal/independent (NI) (or of scale mixture of normal) distributions (Andrews and Mallows, 1974; Lange and Sinsheimer, 1993), called sinh-NI distributions, which contain the sinh-contaminated normal (sinh-CN), sinh-slash (sinh-SL), sinh-Student-t (sinh-St) distributions, among others, all possessing heavier tails than the sinh-normal. Vilca et al. $(2015,2017)$ developed nonlinear regression models under the sinh-NI distributions, which include the log-BS nonlinear regression models proposed by Lemonte and Cordeiro (2009). Recently, Maehara et al. (2017) proposed a generalization of the sinh-NI distribution, in which the skew-normal/independent (SNI) distribution is used in place of the NI distribution. Specifically, consider the r.v. $Z=U^{-1 / 2} Z_{0}$, where $Z_{0}$ follows a standard skew-normal distribution (Azzalini, 1985), denoted by $Z_{0} \sim \mathrm{S} N(0,1, \lambda)$, and $U$ is a positive r.v. independent of $Z_{0}$. Then, a random variable $Y$ stochastically represented in terms of $Z$ by

$$
Y=\mu+\sigma \operatorname{arcsinh}\left(\frac{\alpha}{2} Z\right)=\mu+\sigma \operatorname{arcsinh}\left(\frac{\alpha}{2}\left[U^{-1 / 2} Z_{0}\right]\right)
$$

is said to have a sinh-SNI distribution with shape parameter $\alpha>0$, location parameter $\mu \in$ $\mathbb{R}$, scale parameter $\sigma>0$ and skewness parameter $\lambda \in \mathbb{R}$, where $Z$ follows a standard skewnormal/independent distribution, denoted by $Z \sim \operatorname{SNI}(0,1 ; G)$, where $G(. ; \boldsymbol{\nu})$ is the cumulative distribution function (cdf) of $U$ (indexed by a scalar or vector of parameters $\boldsymbol{\nu}$ ). The distribution 
of $Y$ is denoted by $Y \sim \operatorname{Sinh}-\operatorname{SNI}(\alpha, \mu, \sigma, \lambda ; G)$. Some special cases are, for example, where $\lambda=0$, in which case this distribution reduces to the sinh-NI distribution discussed by Balakrishnan et al. (2009) and Vilca et al. (2017), who extended the sinh-normal distribution by replacing the standard normal distribution with a standard NI distribution. For $U=1$, the sinh-SNI distribution reduces to the skewed sinh-normal distribution; see Leiva et al. (2010) and Santana et al. (2011). Moreover, when $\sigma=2$, the distribution of $Y$ reduces to the log-SNIBS distribution recently proposed by Maehara et al. (2017). The probability density function (pdf) of $Y$ is given by:

$$
f_{Y}(y)=\frac{1}{\sigma} \phi_{\mathrm{SNI}}\left(\xi_{2 y}\right) \xi_{1 y}=\frac{1}{\sigma} \int_{0}^{\infty} \phi\left(\xi_{2 y} ; 0, u^{-1}\right) \Phi\left(\lambda u^{1 / 2} \xi_{2 y}\right) d G(u ; \boldsymbol{\nu}) \xi_{1 y}, y \in \mathbb{R}
$$

where $\phi_{\mathrm{SNI}}(\cdot)$ is the pdf of $Z$ in $(4.1), \xi_{1 y}=\xi_{1}(y ; \alpha, \mu, \sigma)=\frac{2}{\alpha} \cosh \left(\frac{y-\mu}{\sigma}\right), \xi_{2 y}=\xi_{2}(y ; \alpha, \mu, \sigma)$ $=\frac{2}{\alpha} \sinh \left(\frac{y-\mu}{\sigma}\right), \phi\left(. ; \psi, \eta^{2}\right)$ denotes the $N\left(\psi, \eta^{2}\right)$ density function and $\Phi(\cdot)$ is the cdf of the standard normal distribution. Some special cases of the sinh-SNI family are those based on the skew-normal (SN), skew-contaminated normal (SCN), skew-slash (SSL) and skew-Student- $t$ (ST) models, which are called the sinh-SN, sinh-SCN, sinh-SSL and sinh-ST distributions.

Detecting outliers and influential observations and studying the sensitivity to the departure from basic assumptions are important issues in data analysis. Following the pioneering work of Cook $(1977,1986)$, this topic has received a great deal of attention in statistics, and it is still an active area of research in regression models, see for example, Zhu et al. (2001), Zhu and Lee (2001), Xie and Wei (2007) and Lee and Xu (2004), among others. The case-deletion approach is a popular way to assess the individual impact of cases on the estimation (Cook, 1977). Diagnostic measures such as Cook's distance and/or likelihood distance have been applied to various statistical models. The second approach is the local influence approach (Cook, 1986), in which the stability of the estimation outputs with respect to the model inputs is studied via various minor perturbations. When the observed-data log-likelihood function associated with the proposed model is somewhat complex, it is difficult to directly apply the approach of Cook $(1977,1986)$. So, inspired by Zhu and Lee (2001) and Zhu et al. (2001), here we develop the case-deletion measures and the local influence measures in the sinh-SNI regression models on the basis of a $Q$-function.

This paper is organized as follows. Section 4.2 defines briefly the sinh-SNI regression models and describes an EM algorithm for obtaining the maximum likelihood (ML) estimates. Section 4.3 provides a brief sketch of the case-deletion (Zhu et al., 2001) and local influence (Zhu and Lee, 2001) approaches for models with incomplete datasets, and also develops these approaches for the sinh-SNI regression models. Section 4.4 illustrates the proposed methods through simulation 
studies and two examples with real datasets. Finally, concluding remarks are given in Section 4.5.

\subsection{The proposed regression model}

In this section, we present the sinh-SNI nonlinear regression model (sinh-SNI-RM) proposed recently by Maehara et al. (2017), which was inspired by a similar set-up to that developed by Lemonte and Cordeiro (2009) and Vilca et al. (2017). Specifically, this model is defined by:

$$
Y_{i}=f\left(\boldsymbol{\beta} ; \mathbf{x}_{i}\right)+\varepsilon_{i}, i=1, \ldots, n,
$$

where $Y_{i}$ is the response variable, $\mathbf{x}_{i}$ is an $m \times 1$ vector of known explanatory variables associated with the $i$ th observable response $Y_{i}$ and $\mu_{i}(\boldsymbol{\beta})=f\left(\boldsymbol{\beta} ; \mathbf{x}_{i}\right)$ is an injective and twice differentiable function with $\boldsymbol{\beta}=\left(\beta_{1}, \ldots, \beta_{p}\right)$, that is a vector of unknown nonlinear parameters. We assume that $\varepsilon_{i} \sim \operatorname{Sinh}-\operatorname{SNI}(\alpha, 0, \sigma=2, \lambda ; G), i=1, \ldots, n$, are independent. So, from the linearity property, $Y_{i} \sim$ $\operatorname{Sinh}-\operatorname{SNI}\left(\alpha, f\left(\boldsymbol{\beta} ; \mathbf{x}_{i}\right), \sigma=2, \lambda ; G\right)$. Note that when we assume that $\sigma=2$, we are just considering the log-BS nonlinear regression model based on the SNI distribution, which we denote by $Y_{i} \sim$ $\log$-SNIBS $\left(\alpha, f\left(\boldsymbol{\beta} ; \mathbf{x}_{i}\right), \lambda ; G\right)$. The pdf of $Y_{i}$ is given by:

$$
f_{Y_{i}}\left(y_{i}\right)=\frac{1}{2} \phi_{\mathrm{SNI}}\left(\xi_{2 i}\right) \xi_{1 i}
$$

where $\xi_{1 i}=\xi_{1}\left(y_{i} ; \alpha, \boldsymbol{\beta}\right)$ and $\xi_{2 i}=\xi_{2}\left(y_{i} ; \alpha, \boldsymbol{\beta}\right)$ are as in $(4.2)$, with $\sigma=2$ and $\mu_{i}=f\left(\boldsymbol{\beta} ; \mathbf{x}_{i}\right)$ (here we use $\xi_{1 i}$ and $\xi_{2 i}$ instead of $\xi_{1 y_{i}}$ and $\xi_{2 y_{i}}$, respectively).

Remark 5. Before of describing some properties of the nonlinear regression model, we talk about some special cases of the proposed regression model:

(i) For $U=1$ and $\mu_{i}=\mathbf{x}_{i}^{\top} \boldsymbol{\beta}$, we obtain the skew-BS log-linear regression model proposed by Santana et al. (2011);

(ii) for $\lambda=0$, we obtain the nonlinear regression model proposed by Vilca et al. (2015) and Vilca et al. (2017), and for $U=1$, we obtain the nonlinear regression model of Lemonte and Cordeiro (2009);

(iii) for $\lambda=0, U \sim \operatorname{Gamma}(\nu / 2, \nu / 2)$ and $\mu_{i}=\mathbf{x}_{i}^{\top} \boldsymbol{\beta}$, we obtain the linear regression proposed by Paula et al. (2011). 
Remark 6. From the definition of the model in (4.3) and (4.4), we assume that:

$$
Z_{i}=\xi_{2}\left(Y_{i} ; \alpha, \mu, \sigma\right)=\frac{2}{\alpha} \sinh \left(\frac{Y_{i}-\mu_{i}}{\sigma}\right) \sim S N I(0,1 ; G), i=1, \ldots, n
$$

Consequently, its quadratic form follows a generalized $\chi^{2}$ distribution according to properties from the SNI distribution. For example $\xi_{2}^{2}\left(Y_{i} ; \alpha, \mu, \sigma\right) \sim \chi_{1}^{2}$ under the skew-normal distribution and $\xi_{2}^{2}\left(Y_{i} ; \alpha, \mu, \sigma\right) \sim F(1, \nu)$, and so on; see Lange and Sinsheimer (1993) for more information. The random variable

$$
d\left(Y_{i}\right)=\xi_{2}^{2}\left(Y_{i} ; \alpha, \mu_{i}, \sigma\right)
$$

is extremely useful for checking the validity of the model as well as for detecting outliers.

As the observed-data log-likelihood function $\ell(\boldsymbol{\theta})=\sum_{i=1}^{n} \log \left(f_{Y_{i}}\left(y_{i}\right)\right)$ involves complex expressions, so it is very difficult to work directly with $\ell(\boldsymbol{\theta})$, either for the ML estimation or for applying diagnostics analysis procedures. For our proposed nonlinear model, an EM algorithm was developed by Maehara et al. (2017) to perform the ML estimation following the idea of Dempster et al. (1977), which is summarized next. The EM algorithm is a useful tool for finding maximum likelihood (ML) estimates in incomplete-data problems. Each iteration of the EM algorithm has two steps; the expectation step (E-step), where we calculate the conditional expectation of the complete-data log-likelihood given the observed data ( $Q$-function) and the (current) parameter estimate, and the maximization step (M-step), where the (next) parameter estimate is found by maximization of the $Q$-function. This maximization method is considered in the sinh-SNI-RM to find the ML estimates of model parameters. Thus, by using the results in Theorem 5 , the regression model can be expressed hierarchically as:

$$
\begin{aligned}
Y_{i} \mid\left(H_{i}=h_{i}, U_{i}=u_{i}\right) & \stackrel{\text { ind }}{\sim} \sim \operatorname{SHN}\left(\alpha_{\delta, u_{i}}, f\left(\boldsymbol{\beta} ; \mathbf{x}_{i}\right), \sigma=2, \lambda_{h_{i}, u_{i}}\right), \\
H_{i} \mid U_{i}=u_{i} & \stackrel{\text { ind }}{\sim} \sim H N\left(0, u_{i}^{-1}\right), \\
U_{i} & \stackrel{\text { ind }}{\sim} h_{U}\left(u_{i}\right), \quad i=1, \ldots, n,
\end{aligned}
$$

where $\alpha_{\delta, u_{i}}=u_{i}^{1 / 2} \alpha \sqrt{1-\delta^{2}}$ and $\lambda_{h_{i}, u_{i}}=-u_{i}^{1 / 2} \delta h_{i} /\left(\sqrt{1-\delta^{2}}\right)$. We assume that the parameter vector $\boldsymbol{\nu}$ that indexes the pdf $h_{U}(\cdot)$ is known. An optimal value of $\boldsymbol{\nu}$ can then be chosen by using the Schwarz information criterion; see Spiegelhalter et al. (2002) and Lange et al. (1989).

Let $\mathbf{u}=\left(u_{1}, \ldots, u_{n}\right)^{\top}$ be the unobserved data. Then, the complete data are $\mathbf{y}_{c}=\left(\mathbf{y}^{\top}, \mathbf{u}^{\top}\right)^{\top}$ which correspond to the original data $\mathbf{y}=\left(y_{1}, \ldots, y_{n}\right)^{\top}$ augmented with $\mathbf{u}$. Thus, under the 
hierarchical representation given by Equations (4.5)-(4.7), it follows that the complete-data loglikelihood function for $\boldsymbol{\theta}=\left(\alpha, \boldsymbol{\beta}^{\top}, \lambda\right)^{\top}$ given $\mathbf{y}_{c}$ (without the additive constant) can be expressed as:

$$
\ell_{c}\left(\boldsymbol{\theta} \mid \mathbf{y}_{c}\right)=-\frac{n}{2} \log \left(1-\delta^{2}\right)+\sum_{i=1}^{n} \log \left(\xi_{1 i}\right)-\frac{1}{2\left(1-\delta^{2}\right)} \sum_{i=1}^{n} u_{i}\left(\xi_{2 i}-\delta h_{u_{i}}\right)^{2},
$$

where $\xi_{1 i}$ and $\xi_{2 i}$ are as in (4.4). The conditional expectation of the complete-data log-likelihood function has the form:

$$
Q\left(\boldsymbol{\theta} \mid \widehat{\boldsymbol{\theta}}^{(k)}\right)=E\left[\ell_{c}\left(\boldsymbol{\theta} \mid \mathbf{y}_{c}\right)\right]=\sum_{i=1}^{n} Q_{i}\left(\boldsymbol{\theta} \mid \widehat{\boldsymbol{\theta}}^{(k)}\right)
$$

where $\widehat{\boldsymbol{\theta}}^{(k)}=\left(\widehat{\alpha}^{(k)}, \widehat{\boldsymbol{\beta}}^{(k)}, \widehat{\lambda}^{(k)}\right)^{\top}$ denotes the estimate of $\boldsymbol{\theta}$ at the $k$ th iteration and

$$
Q_{i}\left(\boldsymbol{\theta} \mid \widehat{\boldsymbol{\theta}}^{(k)}\right)=-\frac{1}{2} \log \left(1-\delta^{2}\right)+\log \left(\xi_{1 i}^{(k)}\right)-\frac{1}{2\left(1-\delta^{2}\right)}\left(\widehat{\kappa}_{i}^{(k)} \xi_{2 i}^{2(k)}-2 \delta \widehat{h}_{u_{i}}^{(k)} \xi_{2 i}^{2(k)}+\delta^{2}{\widehat{h^{2}}}_{u_{i}}^{(k)}\right)
$$

with the expressions $\widehat{\kappa}_{i}=\mathrm{E}\left[U_{i} \mid \widehat{\boldsymbol{\theta}}^{(k)}, y_{i}\right], \widehat{h}_{u_{i}}=\mathrm{E}\left[U_{i} H_{i} \mid \widehat{\boldsymbol{\theta}}^{(k)}, y_{i}\right]$ and $\widehat{h_{u_{i}}^{2}}=\mathrm{E}\left[U_{i} H_{i}^{2} \mid \widehat{\boldsymbol{\theta}}^{(k)}, y_{i}\right], i=$ $1, \ldots, n$ being obtained from Theorem 6 , which are given by:

$$
\begin{aligned}
\widehat{h}_{u_{i}} & =\widehat{\delta} \widehat{\xi}_{2 i} \widehat{\kappa}_{i}+\widehat{\tau}_{i} \sqrt{1-\widehat{\delta}^{2}} \\
{\widehat{h^{2}}}_{u_{i}} & =\widehat{\delta}^{2} \widehat{\xi}_{2 i}^{2} \widehat{\kappa}_{i}+\left[1-\widehat{\delta}^{2}\right]+\widehat{\delta} \widehat{\xi}_{2 i} \widehat{\tau}_{i} \sqrt{1-\widehat{\delta}^{2}}
\end{aligned}
$$

Hence, with all this information we then have the following ECM algorithm:

E-step. Given $\boldsymbol{\theta}=\widehat{\boldsymbol{\theta}}^{(k)}$, compute $\widehat{\kappa}_{i}^{(k)}, \widehat{h}_{u_{i}}^{(k)}$ and ${\widehat{h^{2}}}_{u_{i}}^{(k)}$, for $i=1, \ldots, n$, using results in (4.10) and (4.11);

CM-step 1. Fix $\widehat{\boldsymbol{\beta}}^{(k)}$ and update $\widehat{\alpha}^{(k)}$ and $\widehat{\delta}^{(k)}$ as

$$
\begin{aligned}
{\widehat{\alpha^{2}}}^{(k+1)} & =\frac{1}{n} \sum_{i=1}^{n} \widehat{\kappa}_{i} \xi_{2 i}^{2}\left(y_{i} ; 1, \widehat{\boldsymbol{\beta}}^{(k)}\right)+\left[1-\widehat{\tau}_{h_{u_{i}}}^{(k)}\right]\left[\frac{\sum_{i=1}^{n} \widehat{h}_{u_{i}}^{(k)} \xi_{2 i}\left(y_{i} ; 1, \widehat{\boldsymbol{\beta}}^{(k)}\right)}{n \widehat{\tau}_{h_{u_{i}}}^{(k)}}\right]^{2} \text { and } \\
\widehat{\delta}^{(k+1)} & =\frac{1}{\widehat{\alpha}^{(k+1)}} \frac{\sum_{i=1}^{n} \widehat{h}_{u_{i}}^{(k)} \xi_{2 i}\left(y_{i} ; 1, \widehat{\boldsymbol{\beta}}^{(k)}\right)}{n \widehat{\tau}_{h_{u_{i}}}^{(k)}}
\end{aligned}
$$

where $\xi_{2 i}\left(y_{i} ; 1, \boldsymbol{\beta}\right)=2 \sinh \left(\frac{y_{i}-f\left(\boldsymbol{\beta} ; \mathbf{x}_{i}\right)}{2}\right)$ and $\widehat{\tau}_{h_{u_{i}}}^{(k)}=(1 / n) \sum_{i=1}^{n}{\widehat{h^{2}}}_{u_{i}}^{(k)}$;

CM-step 2. Fix $\widehat{\alpha}^{(k+1)}$ and $\widehat{\delta}^{(k+1)}$ and update $\widehat{\boldsymbol{\beta}}^{(k)}$ using

$$
\widehat{\boldsymbol{\beta}}^{(k+1)}=\underset{\beta}{\operatorname{argmax}} Q\left(\widehat{\alpha}^{(k+1)}, \boldsymbol{\beta}, \widehat{\delta}^{(k+1)} \mid \widehat{\boldsymbol{\theta}}^{(k)}\right) .
$$


This process is iterated until convergence, i.e., the distance between two successive evaluations of the actual $\log$-likelihood $\ell(\boldsymbol{\theta})$, such as

$$
\left|\ell\left(\widehat{\boldsymbol{\theta}}^{(r+1)}\right)-\ell\left(\widehat{\boldsymbol{\theta}}^{(r)}\right)\right| \text { or }\left|\ell\left(\widehat{\boldsymbol{\theta}}^{(r+1)}\right) / \ell\left(\widehat{\boldsymbol{\theta}}^{(r)}\right)-1\right|
$$

becomes small enough for the desired level of accuracy.

\subsection{Diagnostic analysis}

Influence diagnostic techniques are used to identify influential observations that impact model fit or statistical inference for the assumed statistical model. This topic has received a great deal of attention in statistics, and it is still an active area of research regarding influential observations. In the literature, there are two well-known approaches to detect influential observations. The first approach, the case-deletion approach of Cook (1977) is the most popular one for identifying influential observations. To assess the impact of influential observations on parameter estimates, the metrics that have been used to measure the distance between $\widehat{\boldsymbol{\theta}}_{[j]}$ and $\widehat{\boldsymbol{\theta}}$, are the likelihood distance and Cook's distance. The second approach is a general statistical technique used to assess the stability of the estimation outputs with respect to the model inputs proposed by Cook (1986). Here we consider the case-deletion measures and the local influence diagnostics for BS nonlinear regression models on the basis of the $Q$-function inspired by Zhu et al. (2001), and Zhu and Lee (2001). We first develop the case-deletion measures and then the local influence measures based on some perturbation schemes.

\subsubsection{Case-deletion measures}

Case-deletion is a classic approach to study the effects of dropping the $j$ th case from the dataset. Let $\mathbf{y}_{c}=(\mathbf{y}, \mathbf{u})$ be the augmented dataset, where a quantity with a subscript $[j]$ denotes the original one with the $j$ th observation deleted. The complete data log-likelihood function based on the data with the $j$ th case deleted is denoted by $\ell\left(\boldsymbol{\theta} \mid \mathbf{y}_{c[j]}\right)$. Let $\widehat{\boldsymbol{\theta}}_{[j]}=\left(\widehat{\alpha}_{[j]}, \widehat{\boldsymbol{\beta}}_{[j]}^{\top}, \widehat{\lambda}_{[j]}\right)^{\top}$ be the maximizer of the function $Q_{[j]}(\boldsymbol{\theta} \mid \widehat{\boldsymbol{\theta}})=\mathrm{E}\left[\ell_{c}\left(\boldsymbol{\theta} \mid \mathbf{Y}_{c[j]}\right) \mid \mathbf{y}_{[j]}, \boldsymbol{\theta}=\widehat{\boldsymbol{\theta}}\right]$, where $\widehat{\boldsymbol{\theta}}=\left(\widehat{\alpha}, \widehat{\boldsymbol{\beta}}^{\top}, \widehat{\lambda}\right)^{\top}$ is the ML estimate of $\boldsymbol{\theta}$, and the estimates $\widehat{\boldsymbol{\theta}}_{[j]}$ are obtained by using the EM algorithm based on the remaining $n-1$ observations, with $\widehat{\boldsymbol{\theta}}$ as the initial value. To assess the influence of the $i$ th case on $\widehat{\boldsymbol{\theta}}$, we compare $\widehat{\boldsymbol{\theta}}_{[j]}$ with $\widehat{\boldsymbol{\theta}}$. If $\widehat{\boldsymbol{\theta}}_{[j]}$ is far from $\widehat{\boldsymbol{\theta}}$ in some sense, then the $i$ th case is regarded as influential. Since $\widehat{\boldsymbol{\theta}}_{[j]}$ is needed for every case, the required computational effort can be quite heavy if the sample 
size is large. Hence, to calculate the case-deletion estimate $\widehat{\boldsymbol{\theta}}_{[j]}^{1}$ of $\boldsymbol{\theta}$, Zhu et al. (2001) proposed the following one-step approximation based on the $Q$-function:

$$
\widehat{\boldsymbol{\theta}}_{[j]}^{1}=\widehat{\boldsymbol{\theta}}+[-\ddot{Q}(\widehat{\boldsymbol{\theta}} \mid \widehat{\boldsymbol{\theta}})]^{-1} \dot{Q}_{[j]}(\widehat{\boldsymbol{\theta}} \mid \widehat{\boldsymbol{\theta}}),
$$

where $\ddot{Q}(\widehat{\boldsymbol{\theta}} \mid \widehat{\boldsymbol{\theta}})=\partial^{2} Q(\boldsymbol{\theta} \mid \widehat{\boldsymbol{\theta}}) /\left.\partial \boldsymbol{\theta} \partial \boldsymbol{\theta}^{\top}\right|_{\boldsymbol{\theta}=\widehat{\boldsymbol{\theta}}}$ and $\dot{Q}_{[j]}(\widehat{\boldsymbol{\theta}} \mid \widehat{\boldsymbol{\theta}})=\partial Q_{[j]}(\boldsymbol{\theta} \mid \widehat{\boldsymbol{\theta}}) /\left.\partial \boldsymbol{\theta}\right|_{\boldsymbol{\theta}=\widehat{\boldsymbol{\theta}}}$ are the Hessian matrix and the gradient vector evaluated at $\widehat{\boldsymbol{\theta}}$, respectively. The Hessian matrix is an essential element in the method proposed by Zhu et al. (2001) to obtain the measures for the casedeletion diagnostics. To develop the case-deletion measures, we need to obtain the elements in (4.12), $\dot{Q}_{[j]}(\widehat{\boldsymbol{\theta}} \mid \widehat{\boldsymbol{\theta}})$ and $\ddot{Q}(\widehat{\boldsymbol{\theta}} \mid \widehat{\boldsymbol{\theta}})$, which can be obtained easily from (4.9). The vector $\dot{Q}_{[j]}(\widehat{\boldsymbol{\theta}} \mid \widehat{\boldsymbol{\theta}})=$ $\left(\dot{Q}_{[j] \alpha}(\widehat{\boldsymbol{\theta}} \mid \widehat{\boldsymbol{\theta}}), \dot{Q}_{[j] \boldsymbol{\beta}}(\widehat{\boldsymbol{\theta}} \mid \widehat{\boldsymbol{\theta}})^{\top}, \dot{Q}_{[j] \lambda}(\widehat{\boldsymbol{\theta}} \mid \widehat{\boldsymbol{\theta}})\right)^{\top}$ has its elements given by:

$$
\begin{aligned}
\dot{Q}_{[j] \boldsymbol{\gamma}}(\widehat{\boldsymbol{\theta}} \mid \widehat{\boldsymbol{\theta}}) & =\sum_{i=1, i \neq j}^{n}\left(\frac{1}{\xi_{1 i}} \frac{\partial \xi_{1 i}}{\partial \boldsymbol{\gamma}}-\frac{1+\lambda^{2}}{2} \widehat{\kappa}_{i} \frac{\partial \xi_{2 i}^{2}}{\partial \boldsymbol{\gamma}}+\lambda \sqrt{1+\lambda^{2}} \widehat{h}_{u_{i}} \frac{\partial \xi_{2 i}}{\partial \boldsymbol{\gamma}}\right), \quad \boldsymbol{\gamma}=\boldsymbol{\beta} \quad \text { or } \quad \alpha, \\
\dot{Q}_{[j] \lambda}(\widehat{\boldsymbol{\theta}} \mid \widehat{\boldsymbol{\theta}}) & =\sum_{i=1, i \neq j}^{n}\left(\frac{\lambda}{1+\lambda^{2}}-\lambda \widehat{\kappa}_{i} \xi_{2 i}^{2}+\frac{1+2 \lambda^{2}}{\sqrt{1+\lambda^{2}}} \widehat{h}_{u_{i}} \xi_{2 i}-\widehat{h}_{u_{i}}^{2}\right) .
\end{aligned}
$$

Moreover, the elements of the Hessian matrix $\ddot{Q}(\widehat{\boldsymbol{\theta}} \mid \widehat{\boldsymbol{\theta}})$ are given by:

$$
\begin{aligned}
\frac{\partial Q(\widehat{\boldsymbol{\theta}} \mid \widehat{\boldsymbol{\theta}})}{\partial \boldsymbol{\gamma} \partial \boldsymbol{\tau}^{\top}}= & -\sum_{i=1}^{n}\left(\frac{1}{\xi_{1 i}^{2}} \frac{\partial \xi_{1 i}}{\partial \boldsymbol{\gamma}} \frac{\partial \xi_{1 i}}{\partial \boldsymbol{\tau}^{\top}}-\frac{1}{\xi_{1 i}} \frac{\partial^{2} \xi_{1 i}}{\partial \boldsymbol{\gamma} \partial \boldsymbol{\tau}^{\top}}+\frac{1+\lambda^{2}}{2} \widehat{\kappa}_{i} \frac{\partial^{2} \xi_{2 i}^{2}}{\partial \boldsymbol{\gamma} \partial \boldsymbol{\tau}^{\top}}\right. \\
& \left.-\lambda \sqrt{1+\lambda^{2}} \widehat{h}_{u_{i}} \frac{\partial^{2} \xi_{2 i}}{\partial \boldsymbol{\gamma} \partial \boldsymbol{\tau}^{\top}}\right), \quad \boldsymbol{\gamma}, \boldsymbol{\tau}=\boldsymbol{\beta} \quad \text { or } \quad \alpha, \\
\frac{\partial Q(\widehat{\boldsymbol{\theta}} \mid \widehat{\boldsymbol{\theta}})}{\partial \lambda \partial \boldsymbol{\gamma}}= & -\sum_{i=1}^{n}\left(\lambda \widehat{\kappa}_{i} \frac{\partial \xi_{2 i}^{2}}{\partial \boldsymbol{\gamma}}-\frac{1+2 \lambda^{2}}{\sqrt{1+\lambda^{2}}} \widehat{h}_{u_{i}} \frac{\partial \xi_{2 i}}{\partial \boldsymbol{\gamma}}\right), \quad \boldsymbol{\gamma}=\boldsymbol{\beta} \quad \text { or } \quad \alpha, \\
\frac{\partial Q(\widehat{\boldsymbol{\theta}} \mid \widehat{\boldsymbol{\theta}})}{\partial \lambda \partial \lambda}= & \sum_{i=1}^{n}\left(\frac{1-\lambda^{2}}{\left(1+\lambda^{2}\right)^{2}}-\widehat{\kappa}_{i} \xi_{2 i}^{2}+\frac{\lambda\left(3+2 \lambda^{2}\right)}{\left(1+\lambda^{2}\right)^{3 / 2}} \widehat{h}_{u_{i}} \xi_{2 i}-\widehat{h}_{u_{i}}^{2}\right) .
\end{aligned}
$$

Inspired by the classic case-deletion measures, Cook's distance and the likelihood displacement, Zhu et al. (2001) and Lee and Xu (2004) presented analogous measures based on the $Q$-function.

These measures are:

(i) Generalized Cook's distance: This measure is defined similar to the usual Cook's distance based on the genuine likelihood, and is defined as:

$$
G D_{j}=\left(\widehat{\boldsymbol{\theta}}_{[j]}-\widehat{\boldsymbol{\theta}}\right)^{\top}[-\ddot{Q}(\widehat{\boldsymbol{\theta}} \mid \widehat{\boldsymbol{\theta}})]\left(\widehat{\boldsymbol{\theta}}_{[j]}-\widehat{\boldsymbol{\theta}}\right) .
$$


Upon substituting (4.12) into (4.13), we obtain the approximate distance, given by:

$$
G D_{j}^{1}=\dot{Q}_{[j]}(\widehat{\boldsymbol{\theta}} \mid \widehat{\boldsymbol{\theta}})^{\top}[-\ddot{Q}(\widehat{\boldsymbol{\theta}} \mid \widehat{\boldsymbol{\theta}})]^{-1} \dot{Q}_{[j]}(\widehat{\boldsymbol{\theta}} \mid \widehat{\boldsymbol{\theta}})
$$

(ii) $Q$-distance: This measure of the influence of the $j$ th case is based on the $Q$-distance function, similar to the likelihood distance $L D_{j}$ discussed by Cook (1977), defined as:

$$
Q D_{j}=2\left[Q(\widehat{\boldsymbol{\theta}} \mid \widehat{\boldsymbol{\theta}})-Q\left(\widehat{\boldsymbol{\theta}}_{[j]} \mid \widehat{\boldsymbol{\theta}}\right)\right]
$$

An approximation of the likelihood displacement $Q D_{j}$ is obtained by substituting (4.12) into (4.15), resulting in the following approximation $Q D_{j}^{1}$ of $Q D_{j}$ given by:

$$
Q D_{j}^{1}=2\left[Q(\widehat{\boldsymbol{\theta}} \mid \widehat{\boldsymbol{\theta}})-Q\left(\widehat{\boldsymbol{\theta}}_{[j]}^{1} \mid \widehat{\boldsymbol{\theta}}\right)\right]
$$

Now, if the interest is to consider the influence of the $j$ th case on some subset of parameters, then it can be obtained quite easily as follows: Let $\boldsymbol{\theta}=\left(\boldsymbol{\theta}_{1}^{\top}, \boldsymbol{\theta}_{2}^{\top}\right)^{\top}$ be the vector parameter, where we are interested in discovering the influence of $j$ th case on the estimate of parameter $\boldsymbol{\theta}_{1}$, for example when $\boldsymbol{\theta}_{1}^{\top}=\left(\alpha, \boldsymbol{\beta}^{\top}\right)^{\top}$ is the parameter of the usual regression model and $\boldsymbol{\theta}_{2}=\lambda$ is the skewness parameter. Other cases are $\boldsymbol{\theta}_{1}=\alpha, \boldsymbol{\theta}_{1}=\boldsymbol{\beta}$ or $\boldsymbol{\theta}_{1}=\lambda$. From the expressions of $\dot{Q}_{[j]}(\widehat{\boldsymbol{\theta}} \mid \widehat{\boldsymbol{\theta}})$ and $\ddot{Q}(\widehat{\boldsymbol{\theta}} \mid \widehat{\boldsymbol{\theta}}))$, the generalized Cook's distance for the parameters $\boldsymbol{\theta}_{1}^{\top}=\left(\alpha, \boldsymbol{\beta}^{\top}\right)^{\top}$ and $\lambda$ can be defined as follows:

(1) Both $\alpha$ and $\boldsymbol{\beta}$ are the parameters of interest and $\lambda$ is the nuisance parameter:

$$
G D_{j}^{1}(\alpha, \boldsymbol{\beta})=\dot{Q}_{[j]} \boldsymbol{\theta}_{1}(\widehat{\boldsymbol{\theta}} \mid \widehat{\boldsymbol{\theta}})^{\top}\left[\mathbf{I}_{p+1}, \mathbf{0}\right][-\ddot{Q}(\widehat{\boldsymbol{\theta}} \mid \widehat{\boldsymbol{\theta}})]^{-1}\left[\mathbf{I}_{p+1}, \mathbf{0}\right]^{\top} \dot{Q}_{[j]} \boldsymbol{\theta}_{1}(\widehat{\boldsymbol{\theta}} \mid \widehat{\boldsymbol{\theta}}),
$$

where $\dot{Q}_{[j]} \boldsymbol{\theta}_{1}(\widehat{\boldsymbol{\theta}} \mid \widehat{\boldsymbol{\theta}})=\left(\dot{Q}_{[j] \alpha}(\widehat{\boldsymbol{\theta}} \mid \widehat{\boldsymbol{\theta}}), \dot{Q}_{[j]}(\widehat{\boldsymbol{\beta}}(\widehat{\boldsymbol{\theta}} \mid \widehat{\boldsymbol{\theta}}))^{\top}\right.$, with $\boldsymbol{\theta}_{1}=\left(\alpha, \boldsymbol{\beta}^{\top}\right)^{\top}$

(2) $\lambda$ is the parameter of interest and both $\alpha$ and $\boldsymbol{\beta}$ are nuisance parameters:

$$
\begin{aligned}
G D_{j}^{1}(\lambda) & =\dot{Q}_{[j] \lambda}(\widehat{\boldsymbol{\theta}} \mid \widehat{\boldsymbol{\theta}})^{\top} \mathbf{b}_{2}^{\top}[-\ddot{Q}(\widehat{\boldsymbol{\theta}} \mid \widehat{\boldsymbol{\theta}})]^{-1} \mathbf{b}_{2} \dot{Q}_{[j] \lambda}(\widehat{\boldsymbol{\theta}} \mid \widehat{\boldsymbol{\theta}}), \\
& =\left(\dot{Q}_{[j] \lambda}(\widehat{\boldsymbol{\theta}} \mid \widehat{\boldsymbol{\theta}})\right)^{2} \mathbf{b}_{2}^{\top}[-\ddot{Q}(\widehat{\boldsymbol{\theta}} \mid \widehat{\boldsymbol{\theta}})]^{-1} \mathbf{b}_{2},
\end{aligned}
$$

where $\dot{Q}_{[j] \lambda}(\widehat{\boldsymbol{\theta}} \mid \widehat{\boldsymbol{\theta}})$ is the third element of $\dot{Q}_{[j]}(\widehat{\boldsymbol{\theta}} \mid \widehat{\boldsymbol{\theta}})$ and $\mathbf{b}_{2}$ is the $(p+2) \times 1$ vector with one at the $(p+2)$ th position. 


\subsubsection{The local influence approach}

Consider a perturbation vector $\boldsymbol{\omega}$ varying in an open region $\boldsymbol{\Omega} \in \mathbb{R}^{q}$. Let $\ell_{c}\left(\boldsymbol{\theta}, \boldsymbol{\omega} \mid \mathbf{y}_{c}\right), \boldsymbol{\theta} \in$ $\mathbb{R}^{p}$ be the complete data log-likelihood of the perturbed model. We assume there is a $\boldsymbol{\omega}_{0}$ such that $\ell_{c}\left(\boldsymbol{\theta}, \boldsymbol{\omega}_{0} \mid \mathbf{y}_{c}\right)=\ell_{c}\left(\boldsymbol{\theta} \mid \mathbf{y}_{c}\right)$ for all $\boldsymbol{\theta}$. Let $\widehat{\boldsymbol{\theta}}_{\boldsymbol{\omega}}$ be the maximizer of the function $Q(\boldsymbol{\theta}, \boldsymbol{\omega} \mid \widehat{\boldsymbol{\theta}})=$ $E\left[\ell_{c}\left(\boldsymbol{\theta}, \boldsymbol{\omega} \mid \mathbf{y}_{c}\right) \mid \mathbf{y}, \widehat{\boldsymbol{\theta}}\right]$. Then the influence graph is defined as $\boldsymbol{\alpha}(\boldsymbol{\omega})=\left(\boldsymbol{\omega}^{\top}, f_{Q}(\boldsymbol{\omega})\right)^{\top}$, where $f_{Q}(\boldsymbol{\omega})$ is the $Q$-displacement function defined as:

$$
f_{Q}(\boldsymbol{\omega})=2\left[Q(\widehat{\boldsymbol{\theta}} \mid \widehat{\boldsymbol{\theta}})-Q\left(\widehat{\boldsymbol{\theta}}_{\boldsymbol{\omega}} \mid \widehat{\boldsymbol{\theta}}\right)\right]
$$

Following the approach developed in Zhu and Lee (2001), the normal curvature $C_{f_{Q}, \mathbf{d}}$, of $\boldsymbol{\alpha}(\boldsymbol{\omega})$ at $\boldsymbol{\omega}_{0}$ in the direction of a unit vector $\mathbf{d}$ can be used to summarize the local behavior of the $Q$-displacement function. It can be shown that:

$$
C_{f_{Q}, \mathbf{d}}(\boldsymbol{\theta})=-2 \mathbf{d}^{\top} \ddot{Q} \boldsymbol{\omega}_{0} \mathbf{d}=2 \mathbf{d}^{\top} \boldsymbol{\Delta}_{\boldsymbol{\omega}_{0}}^{\top}\{-\ddot{Q}(\widehat{\boldsymbol{\theta}} \mid \widehat{\boldsymbol{\theta}})\}^{-1} \boldsymbol{\Delta}_{\boldsymbol{\omega}_{0}} \mathbf{d}^{\top}
$$

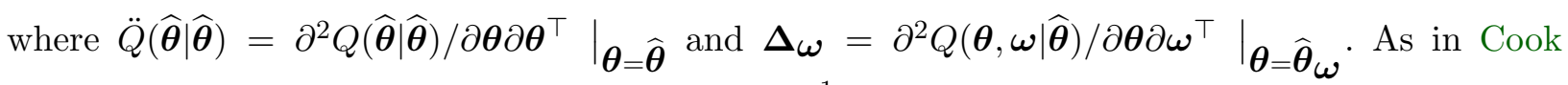
(1986), the expression $-\ddot{Q} \boldsymbol{\omega}_{0}=\boldsymbol{\Delta}_{\boldsymbol{\omega}_{0}}^{\top}\{-\ddot{Q}(\widehat{\boldsymbol{\theta}} \mid \widehat{\boldsymbol{\theta}})\}^{-1} \boldsymbol{\Delta}_{\boldsymbol{\omega}_{0}}$ is fundamental to detect influential observations. When it is of interest to assess the influence on a subset $\boldsymbol{\theta}_{1}$ of $\boldsymbol{\theta}=\left(\boldsymbol{\theta}_{1}, \boldsymbol{\theta}_{2}\right)^{\top}$, the curvature in the direction $\mathbf{d}$ is given by:

$$
C_{f_{Q}, \mathbf{d}}\left(\boldsymbol{\theta}_{1}\right)=2 \mathbf{d}^{\top} \Delta_{\boldsymbol{\omega}_{0}}^{\top}\left(-\ddot{Q}(\widehat{\boldsymbol{\theta}} \mid \widehat{\boldsymbol{\theta}})^{-1}-B_{22}\right) \Delta_{\boldsymbol{\omega}_{0}} \mathbf{d}^{\top}
$$

where

$$
B_{22}=\left(\begin{array}{cc}
\mathbf{0} & \mathbf{0} \\
\mathbf{0} & -\ddot{Q}_{22}(\widehat{\boldsymbol{\theta}} \mid \widehat{\boldsymbol{\theta}})^{-1}
\end{array}\right)
$$

and $\ddot{Q}_{22}(\widehat{\boldsymbol{\theta}} \mid \widehat{\boldsymbol{\theta}})$ is obtained from the partition of $\ddot{Q}(\widehat{\boldsymbol{\theta}} \mid \widehat{\boldsymbol{\theta}})$ according to the partition of $\boldsymbol{\theta}$. A clear picture of $-\ddot{Q} \boldsymbol{\omega}_{0}$ (a symmetric matrix) is given by its spectral decomposition

$$
-2 \ddot{Q} \boldsymbol{\omega}_{0}=\sum_{k=1}^{n} \lambda_{k} \mathbf{e}_{k} \mathbf{e}_{k}^{\top},
$$

where $\left(\lambda_{1}, \mathbf{e}_{1}\right), \ldots,\left(\lambda_{n}, \mathbf{e}_{n}\right)$ are the eigenvalue-eigenvector pairs of the matrix $-2 \ddot{Q}_{\boldsymbol{\omega}_{0}}$, with $\lambda_{1} \geq$ $\ldots \geq \lambda_{q}>\lambda_{q+1}=\ldots=\lambda_{n}=0$ and $\mathbf{e}_{1}, \ldots, \mathbf{e}_{n}$ are elements of the associated orthonormal basis. Zhu and Lee (2001) proposed to inspect all eigenvectors corresponding to nonzero eigenvalues for 
more revealing information, but this can be computationally intensive for large $n$. Following Zhu and Lee (2001) and Lu and Song (2006), we consider an aggregated contribution vector of all eigenvectors corresponding to nonzero eigenvalues. Starting with some notation, let $\bar{\lambda}_{k}=\lambda_{k} /\left(\lambda_{1}+\right.$ $\left.\ldots+\lambda_{k}\right), \mathbf{e}_{k}^{2}=\left(e_{k 1}^{2}, \ldots, e_{k n}^{2}\right)$ and

$$
M(0)=\sum_{k=1}^{q} \bar{\lambda}_{k} \mathbf{e}_{k}^{2} .
$$

Hence, the assessment of influential cases is based on $\left\{M(0)_{l}, l=1, \ldots, n\right\}$ and one can obtain $M(0)_{l}$ via $B_{f_{Q}, \mathbf{u}_{l}}=-2 \mathbf{u}_{l}^{\top} \ddot{Q} \boldsymbol{\omega}_{0} \mathbf{u}_{l} / \operatorname{tr}\left[-2 \ddot{Q} \boldsymbol{\omega}_{0}\right]$, where $\mathbf{u}_{l}$ is a column vector in $\mathbb{R}^{n}$ with the $l$ th entry equal to one and all other entries zero. Refer to Zhu and Lee (2001) for other theoretical properties of $B_{f_{Q}, \mathbf{u}_{l}}$, such as invariance under reparameterization of $\boldsymbol{\theta}$. Additionally, Lee and Xu (2004) propose to use $1 / n+c^{*} S M(0)$ as a benchmark to regard the $l$ th case as influential, where $c^{*}$ is an arbitrary constant (depending on the real application) and $S M(0)$ is the standard deviation of $\left\{M(0)_{l}, l=1, \ldots, n\right\}$.

Finally, following the idea of Verbeke and Molenberghs (2000), we consider another important direction given by $\mathbf{d}=\mathbf{d}_{k q}$ which corresponds to a $q \times 1$ vector with one in the $k$ th position and all other entries zero. In that case, the normal curvature is called the total local influence of the $k$ th observation, which is given by:

$$
C_{k}=\mathbf{d}_{k q}^{\top} \boldsymbol{\Delta}_{\omega_{0}}^{\top}\{-\ddot{Q}(\widehat{\boldsymbol{\theta}} \mid \widehat{\boldsymbol{\theta}})\}^{-1} \boldsymbol{\Delta}_{\omega_{0}} \mathbf{d}_{k q}, \quad k=1, \ldots, q
$$

Moreover, the $k$ th term is an influential observation if $C_{k}$ is larger than the cutoff value $c^{*}=$ $\sum_{k=1}^{q} C_{k} / q$

Now, we are ready to evaluate the matrix $\Delta_{\boldsymbol{\omega}_{0}}$ under three different perturbation schemes: case weights perturbation, response perturbation and explanatory perturbation. Each perturbation scheme has the partitioned form of $\boldsymbol{\Delta}_{\boldsymbol{\omega}_{0}}=\left(\boldsymbol{\Delta}_{\alpha}^{\top}, \boldsymbol{\Delta}_{\beta}^{\top}, \boldsymbol{\Delta}_{\lambda}^{\top}\right)^{\top}$.

\section{Perturbation schemes}

We consider three different perturbation schemes, case weight perturbation, response perturbation and explanatory perturbation, which are discussed with detail.

\section{(i) Case weight perturbation}

Let $\boldsymbol{\omega}=\left(\omega_{1}, \ldots, \omega_{n}\right)$ be an $n \times 1$ dimensional vector with $\boldsymbol{\omega}_{0}=(1, \ldots, 1)^{\top} \in \mathbb{R}^{n}$. The $Q$-function 
for the perturbed model is defined by $Q(\boldsymbol{\theta}, \boldsymbol{\omega} \mid \widehat{\boldsymbol{\theta}})=\sum_{i=1}^{n} \omega_{i} Q_{i}(\widehat{\boldsymbol{\theta}} \mid \widehat{\boldsymbol{\theta}})$. In this case, the elements of the matrix $\boldsymbol{\Delta}_{\boldsymbol{\omega}_{0}}$ are given by

$$
\begin{aligned}
\boldsymbol{\Delta}_{i \gamma} & =\frac{1}{\xi_{1 i}} \frac{\partial \xi_{1 i}}{\partial \boldsymbol{\gamma}}-\frac{1+\lambda^{2}}{2} \widehat{\kappa}_{i} \frac{\partial \xi_{2 i}^{2}}{\partial \boldsymbol{\gamma}}+\lambda \sqrt{1+\lambda^{2}} \widehat{h}_{u_{i}} \frac{\partial \xi_{2 i}}{\partial \boldsymbol{\gamma}}, \quad \boldsymbol{\gamma}=\boldsymbol{\beta} \text { or } \alpha, \\
\boldsymbol{\Delta}_{i \lambda} & =\frac{\lambda}{1+\lambda^{2}}-\lambda \widehat{\kappa}_{i} \xi_{2 i}^{2}+\frac{1+2 \lambda^{2}}{\sqrt{1+\lambda^{2}}} \widehat{h}_{u_{i}} \xi_{2 i}-\lambda \widehat{h}_{u_{i}}^{2} .
\end{aligned}
$$

(ii) Response perturbation

A perturbation of the response variables $\mathbf{Y}=\left(Y_{1}, \ldots, Y_{n}\right)^{\top}$ is introduced by replacing $Y_{i}$ by $Y_{\omega_{i}}=Y_{i}+\omega_{i} S_{y}$, where $S_{y}$ is the standard deviation of $\mathbf{Y}$. The perturbed $Q$-function is given by $Q(\boldsymbol{\theta}, \boldsymbol{\omega} \mid \widehat{\boldsymbol{\theta}})$, switching $Y_{\omega_{i}}$ with $Y_{i}$ and $\boldsymbol{\omega}=\left(\omega_{1}, \ldots, \omega_{n}\right)^{\top}$. Under this perturbation scheme, the vector $\boldsymbol{\omega}_{0}=\mathbf{0}$ and the matrix $\boldsymbol{\Delta}_{\boldsymbol{\omega}_{0}}$ are expressed as:

$$
\begin{aligned}
& \boldsymbol{\Delta}_{i \gamma}=-\frac{S_{y}}{2} \frac{\xi_{2 i}}{\xi_{1 i}^{2}} \frac{\partial \xi_{1 i}}{\partial \gamma}+\frac{1}{\xi_{1 i}} \frac{\partial^{2} \xi_{1 i}}{\partial \gamma \partial \omega_{i}}-\frac{1+\lambda^{2}}{2} \widehat{\kappa}_{i} \frac{\partial^{2} \xi_{2 i}^{2}}{\partial \gamma \partial \omega_{i}}+\lambda \sqrt{1+\lambda^{2}} \widehat{h}_{u_{i}} \frac{\partial^{2} \xi_{2 i}}{\partial \gamma \partial \omega_{i}}, \\
& \boldsymbol{\gamma}=\boldsymbol{\beta} \quad \text { or } \quad \alpha, \\
& \boldsymbol{\Delta}_{i \lambda}=-\lambda S_{y} \widehat{\kappa}_{i} \xi_{1 i} \xi_{2 i}+\frac{S_{y}\left(1+2 \lambda^{2}\right)}{2 \sqrt{1+\lambda^{2}}} \widehat{h}_{u_{i}} \xi_{1 i} .
\end{aligned}
$$

where

$$
\begin{aligned}
\frac{\partial \xi_{1 i}}{\partial \alpha} & =-\frac{1}{\alpha} \xi_{1 i}, \frac{\partial \xi_{1 i}}{\partial \boldsymbol{\beta}}=-\frac{1}{2} \xi_{2 i} \frac{\partial \mu_{i}}{\partial \boldsymbol{\beta}}, \quad \frac{\partial^{2} \xi_{1 i}}{\partial \alpha \partial \omega_{i}}=-\frac{S_{y}}{2 \alpha} \xi_{2 i}, \frac{\partial^{2} \xi_{1 i}}{\partial \boldsymbol{\beta} \partial \omega_{i}}=-\frac{S_{y}}{4} \xi_{1 i} \frac{\partial \mu_{i}}{\partial \boldsymbol{\beta}} \\
\frac{\partial^{2} \xi_{2 i}^{2}}{\partial \alpha \partial \omega_{i}} & =-\frac{2}{\alpha} S_{y} \xi_{1 i} \xi_{2 i}, \frac{\partial^{2} \xi_{2 i}^{2}}{\partial \boldsymbol{\beta} \partial \omega_{i}}=-\frac{S_{y}}{2}\left(\xi_{1 i}^{2}+\xi_{2 i}^{2}\right) \frac{\partial \mu_{i}}{\partial \boldsymbol{\beta}}, \quad \frac{\partial^{2} \xi_{2 i}}{\partial \boldsymbol{\beta} \partial \omega_{i}}=-\frac{S_{y}}{4} \xi_{2 i} \frac{\partial \mu_{i}}{\partial \boldsymbol{\beta}} .
\end{aligned}
$$

(iii) Explanatory perturbation

We are interested in perturbing a specific explanatory variable. Under this condition we have the perturbed explanatory variable $\mathbf{x}_{\omega_{i} p}=\mathbf{x}_{i p}+S_{x} \omega_{i}$, for $i=1, \ldots, n$, where $S_{x}$ is the standard deviation of the explanatory variable $\mathbf{x}_{p}$ and $\boldsymbol{\omega}_{0}=\mathbf{0}$. The perturbed Q-function is given by $Q(\boldsymbol{\theta}, \boldsymbol{\omega} \mid \widehat{\boldsymbol{\theta}})$, switching $\mathbf{x}_{\omega_{i} p}$ with $\mathbf{x}_{i p}$. The $(p+2) \times n$ matrix $\boldsymbol{\Delta}_{\boldsymbol{\omega}_{0}}$ is given by:

$$
\begin{aligned}
\boldsymbol{\Delta}_{i \gamma}= & \frac{1}{2} \frac{\xi_{2 i}}{\xi_{1 i}^{2}} \frac{\partial \mu_{i}}{\partial \omega_{i}} \frac{\partial \xi_{1 i}}{\partial \gamma}+\frac{1}{\xi_{1 i}} \frac{\partial^{2} \xi_{1 i}}{\partial \gamma \partial \omega_{i}}-\frac{1+\lambda^{2}}{2} \widehat{\kappa}_{i} \frac{\partial^{2} \xi_{2 i}^{2}}{\partial \gamma \partial \omega_{i}}+\lambda \sqrt{1+\lambda^{2}} \widehat{h}_{u_{i}} \frac{\partial^{2} \xi_{2 i}}{\partial \gamma \partial \omega_{i}}, \\
& \boldsymbol{\gamma}=\boldsymbol{\beta} \text { or } \quad \alpha, \\
\boldsymbol{\Delta}_{i \lambda}= & -\lambda \widehat{\kappa}_{i} \xi_{1 i} \xi_{2 i} \frac{\partial \mu_{i}}{\partial \omega_{i}}-\frac{1+2 \lambda^{2}}{2 \sqrt{1+\lambda^{2}}} \widehat{h}_{u_{i}} \xi_{1 i} \frac{\partial \mu_{i}}{\partial \omega_{i}}
\end{aligned}
$$


where

$$
\begin{aligned}
\frac{\partial^{2} \xi_{1 i}}{\partial \alpha \partial \omega_{i}} & =\frac{1}{2 \alpha} \xi_{2 i} \frac{\partial \mu_{i}}{\partial \omega_{i}}, \frac{\partial^{2} \xi_{1 i}}{\partial \boldsymbol{\beta} \partial \omega_{i}}=-\frac{1}{2}\left(\xi_{2 i} \frac{\partial^{2} \mu_{i}}{\partial \boldsymbol{\beta} \partial \omega_{i}}-\frac{\xi_{1 i}}{2} \frac{\partial \mu_{i}}{\partial \omega_{i}} \frac{\partial \mu_{i}}{\partial \boldsymbol{\beta}}\right), \\
\frac{\partial^{2} \xi_{2 i}^{2}}{\partial \alpha \partial \omega_{i}} & =\frac{2}{\alpha} \xi_{1 i} \xi_{2 i} \frac{\partial \mu_{i}}{\partial \omega_{i}}, \frac{\partial^{2} \xi_{2 i}^{2}}{\partial \boldsymbol{\beta} \partial \omega_{i}}=\frac{1}{2}\left(\xi_{1 i}^{2}+\xi_{2 i}^{2}\right) \frac{\partial \mu_{i}}{\partial \omega_{i}} \frac{\partial \mu_{i}}{\partial \boldsymbol{\beta}}-\xi_{1 i} \xi_{2 i} \frac{\partial^{2} \mu_{i}}{\partial \boldsymbol{\beta} \partial \omega_{i}}, \\
\frac{\partial^{2} \xi_{2 i}}{\partial \boldsymbol{\beta} \partial \omega_{i}} & =-\frac{1}{2}\left(\xi_{1 i} \frac{\partial^{2} \mu_{i}}{\partial \boldsymbol{\beta} \partial \omega_{i}}-\frac{\xi_{2 i}}{2} \frac{\partial \mu_{i}}{\partial \boldsymbol{\beta}} \frac{\partial \mu_{i}}{\partial \omega_{i}}\right), \frac{\partial^{2} \xi_{2 i}}{\partial \alpha \partial \omega_{i}}=\frac{1}{2 \alpha} \xi_{1 i} \frac{\partial \mu_{i}}{\partial \omega_{i}}
\end{aligned}
$$

\subsection{Numerical application}

In this section, a simulation study and a real example are presented to illustrate the performance of the method. First, we carry out a numerical illustration with simulated data. Finally, we analyze a real dataset to illustrate the usefulness of the proposed method.

\subsubsection{Simulation studies}

In order to evaluate the capacity of the method to detect atypical data and the sensitivity of the ML estimates under alternative distributions than the sinh-SN one, we conducted a small simulation study. We considered four simulated datasets from the regression models defined in (4.3). That is:

$$
Y_{i}=\beta_{0}+\beta_{1} x_{i}+\varepsilon_{i},
$$

where the parameter values are $\alpha=0.5, \beta_{0}=1, \beta_{1}=-2$ and $\lambda=3, i=1, \ldots, 100$. Following the idea of Ortega et al. (2003) and Santana et al. (2011), for the dataset from the proposed linear regression model in (4.22) is introduced outlying observations are introduced under the explanatory and response perturbations.

For the explanatory perturbation, after generating $x_{i}$ from the $U(0,1)$, we perturb the maximum value of the sample, $x_{\max } \longleftarrow x_{\max }+0.2 \sqrt{\left(\mathbf{x}^{\top} \mathbf{x}\right)}$, where $\mathbf{x}=\left(x_{i}, \ldots, x_{100}\right)^{\top}$. In this case, the atypical observation (in $\mathbf{x}$ ) corresponds to individual \#82. Under this type of perturbation, we consider case-weight perturbation and explanatory variables perturbation. In addition, we also consider the response perturbation to individual \#82 as follows: $y_{82} \longleftarrow y_{82}+0.4 \sqrt{\left(\mathbf{y}^{\top} \mathbf{y}\right)}$, where $\mathbf{y}=\left(y_{i}, \ldots, y_{100}\right)^{\top}$. Following our local influence approach, we hope to identify the outlying observations. The results in Figure 4.1 reports the index plots of $M(0)$ for the case-weight perturbation, response and explanatory perturbations, respectively, with the benchmark computed for $c^{*}=5$. Under all perturbation schemes, we notice that observation \#82 is an influential observation only in the sinh-SN case. This confirms the high sensitivity of the ML estimates in the presence of atyp- 
ical observations when the sinh-SN model is fitted. Figure 4.1 also shows that the ML estimates are more robust in the presence of atypical observations, mainly when the sinh-ST, sinh-SSL and sinh-SCN are used, since the perturbed observation was not detected.
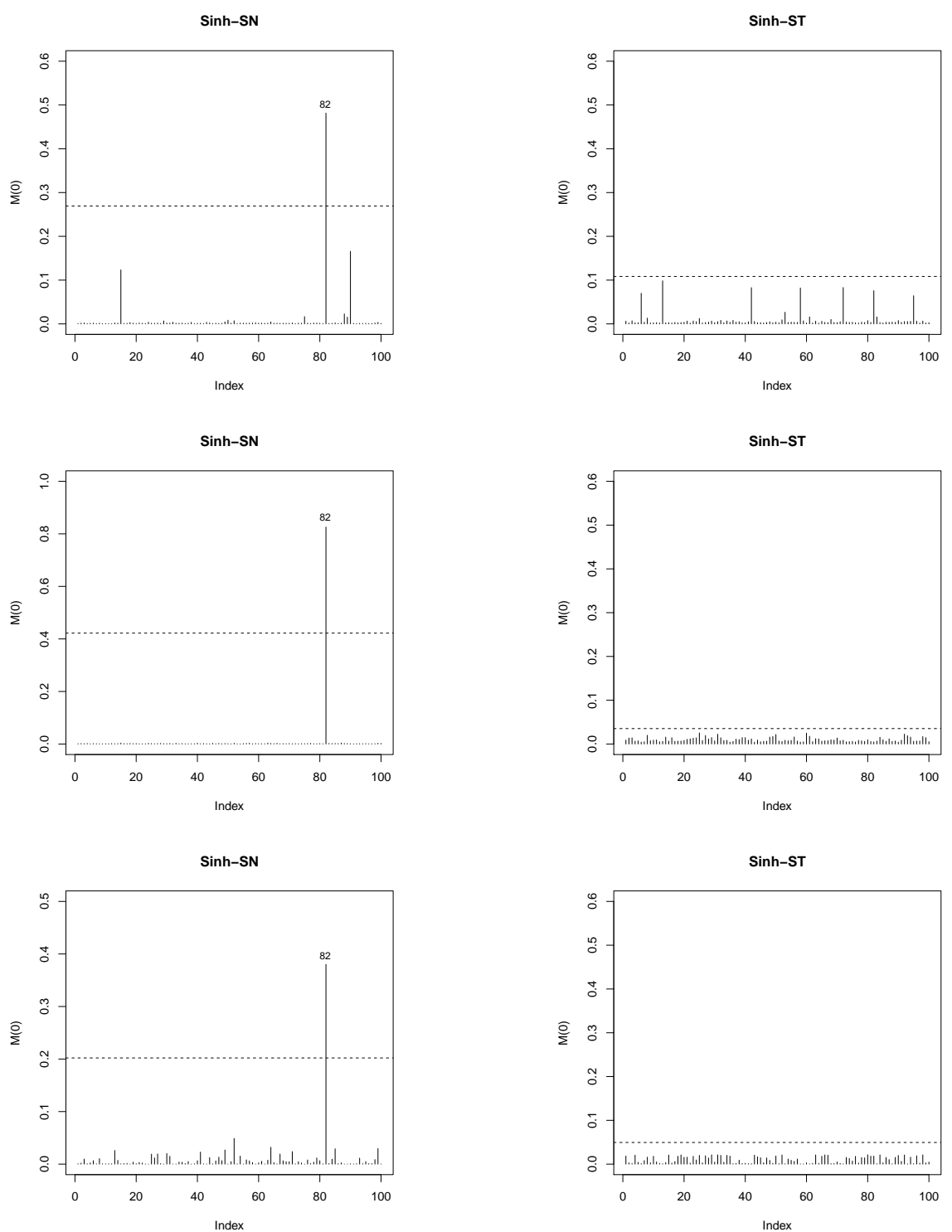

Figure 4.1: Simulated data. Index plot of $M(0)$ in the case weights perturbation using sinh-SN and sinh-ST models.

\subsubsection{Illustrative examples}

For illustration, we consider two datasets, the first on the life of a metal pieces in cycles to failure (biaxial dataset). These data correspond to 46 metal pieces that were tested until failure. The variables considered in this study are: the number of cycles until to failure $(N)$ and the work per cycle (in MJ / $\mathrm{m}^{3}$ ), which was analyzed by Rieck and Nedelman (1991), and afterward by Santana et al. (2011) and Lemonte and Cordeiro (2009). The second dataset (Martin Marietta dataset) 
was previously analyzed by Butler et al. (1990), Azzalini and Capitanio (2003) and Ferreira et al. (2015) (analyzed under an asymmetrical model).

\section{Biaxial fatigue dataset}

This dataset was considered by Lemonte and Cordeiro (2009) for estimation and by Lemonte and Patriota (2011) for diagnostic analysis by considering a log-BS nonlinear regression model

$$
Y_{i}=\beta_{1} x_{i}^{\beta_{2}}+\varepsilon_{i} \quad i=1, \ldots, 46,
$$

where $Y_{i}=\log \left(N_{i}\right)$ is the logarithm of the number of cycles, $x_{i}$ the $i$ th value of the work per cycle, $\beta_{1}$ and $\beta_{2}$ the regression coefficients, and $\varepsilon_{i} \sim \operatorname{Sinh}-\mathrm{N}(\alpha, 0, \sigma=2)$ or $\varepsilon_{i} \sim \log$-BS $(\alpha, 0)$ (symmetric model). We propose to consider $\varepsilon_{i} \sim \operatorname{Sinh}-\operatorname{SNI}(\alpha, 0, \sigma=2 ; G)$ (asymmetric model), based on SNNLM, ST-NLM, SCN-NLM and SSL-NLM. Table 4.1 provides the ML estimates of the model parameters besides their corresponding standard errors (SE). Moreover, the log-likelihood function of the ML estimates and information selection criteria such as AIC, BIC and EDC (sinh-NI-NLM, in parentheses) are reported, which indicate that the sinh-SNI-NLM models have a significantly better fit than sinh-NI-NLM models, specifically the sinh-SN-NLM. Replacing the ML estimates of $\boldsymbol{\theta}$ in $d\left(Y_{i}\right)$ defined in Remark 6, we use it to test the goodness of fit, as can be seen in Figure 4.2, which displays simulated envelopes where lines represent the 5th percentile, the mean and the 95th percentile of 200 simulated points for each observation. For more details see the Appendix (special case). The results in the figure are in agreement with the results in Table 4.2, which allow us to conclude that the sinh-SN-NLM produces a better fit to the current data than sinh-normal models, with the best being the SN-NLM. 
Table 4.1: Biaxial dataset: ML estimates for the four sinh-SNI-NLMs, the log-likelihood values and information criteria values (sinh-NI-NLMs in parentheses).

\begin{tabular}{c|cc|cc|cc|cc}
\hline \hline \multirow{2}{*}{ Parameter } & \multicolumn{2}{|c|}{ Sinh-SN } & \multicolumn{2}{|c|}{ Sinh-ST } & \multicolumn{2}{c|}{ Sinh-SCN } & \multicolumn{2}{c}{ Sinh-SSL } \\
& Estimate & SE & Estimate & SE & Estimate & SE & Estimate & SE \\
\hline$\alpha$ & 0.6946 & 0.1263 & 0.6720 & 0.1319 & 0.6130 & 0.1160 & 0.6408 & 0.1214 \\
$\beta_{1}$ & 16.5663 & 0.7420 & 16.5806 & 0.7442 & 16.5782 & 0.7502 & 16.5587 & 0.7076 \\
$\beta_{2}$ & -0.2495 & 0.0119 & -0.2497 & 0.0117 & -0.2496 & 0.0119 & -0.2503 & 0.0116 \\
$\lambda$ & -6.0551 & 7.5305 & -6.1506 & 8.1610 & -6.2740 & 8.3035 & -4.7967 & 4.9544 \\
$\nu$ & - & - & 16.4 & - & 0.5 & - & 19.2 & - \\
$\gamma$ & - & - & - & - & 0.6 & - & - & - \\
\hline$l(\widehat{\boldsymbol{\theta}})$ & $-20.1796(-22.7458)$ & $-20.6515(-23.4123)$ & $-20.4070(-23.0894)$ & $-20.3857(-22.7824)$ \\
AIC & $48.3593(51.4917)$ & $49.3031(52.8247)$ & $48.8140(52.1788)$ & $48.7715(51.5648)$ \\
BIC & $55.6738(56.9776)$ & $56.6177(58.3106)$ & $56.1286(57.6647)$ & $56.0860(57.0507)$ \\
EDC & $45.7851(49.5611)$ & $46.7290(50.8941)$ & $46.2399(50.2482)$ & $46.1973(49.6342)$ \\
\hline \hline
\end{tabular}
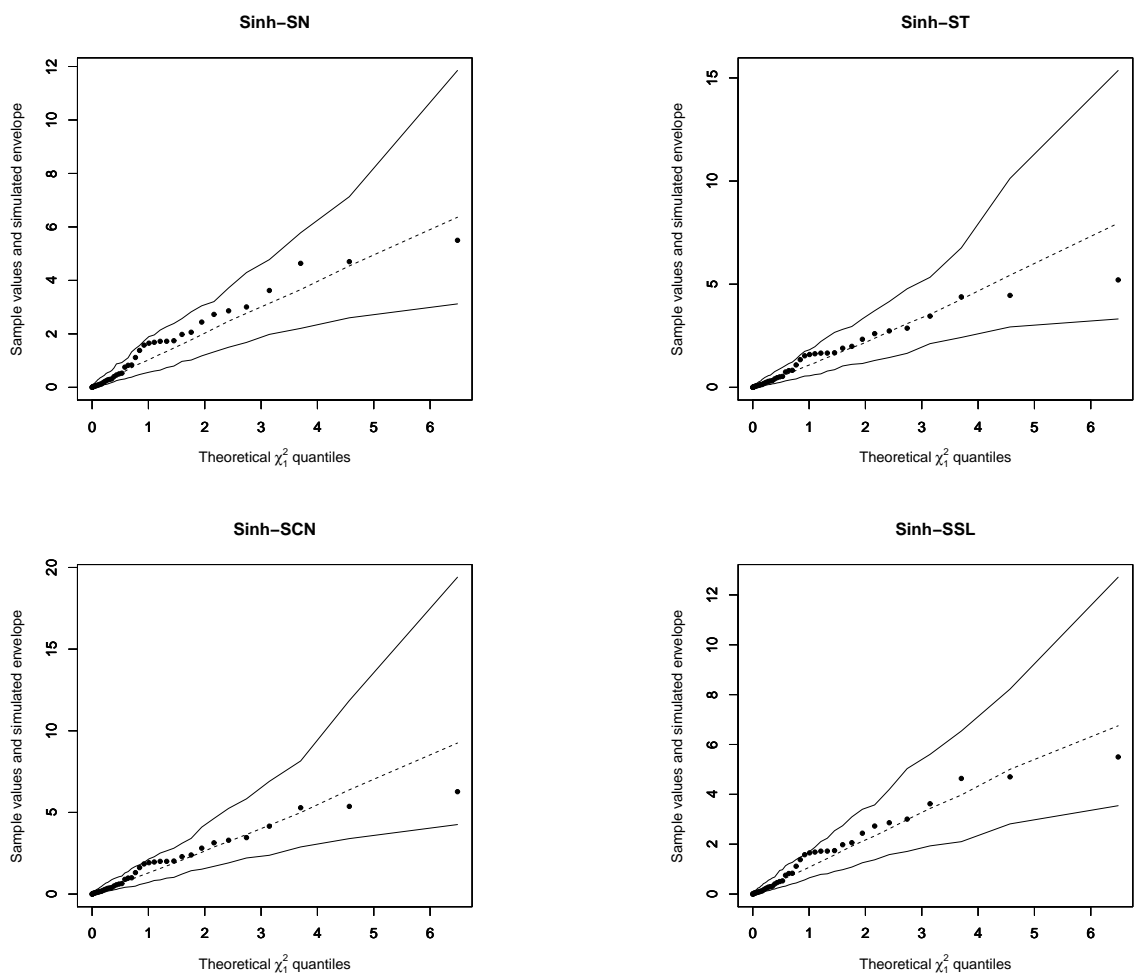

Figure 4.2: Biaxial dataset: Simulated envelopes for sinh-SNI-NLM based on SN, ST, SCN and SSL distributions.

First, we identify outlying observations in the fitted model based on case-deletion measures, the $Q$-distance and generalized Cook distance, whose scatter plot is displayed in Figure 4.3 (left). Also, Figure 4.3 (right) shows the scatter plot of $D_{j}(\lambda)$ vs $D_{j}(\alpha, \beta)$. From this figure note that observation \#4 is potentially influential on the parameter estimates. Second, diagnostic measures based on the local influence are applied to detect outlying observations, using the proposed perturbation schemes. 
Under these perturbation schemes, we obtained the values of $M(0)$ that are provided in the figures. The horizontal lines are the benchmarks, with $c^{*}=1$. So, under these perturbation schemes, observations \#1, \#3, \#4, \#5, \#12 and \#32 are potentially influential on the parameter estimates $\boldsymbol{\theta}$, whose results are provided in Figure 4.4 where observations \#1, \#3, \#5 and \#12 are more influential under response perturbation and work variable perturbation, and observation \#4 is just influential under case-weight perturbation and response perturbation. Most of those observations were detected as influential observations in the linear log-BS regression model discussed by Santana et al. (2011).
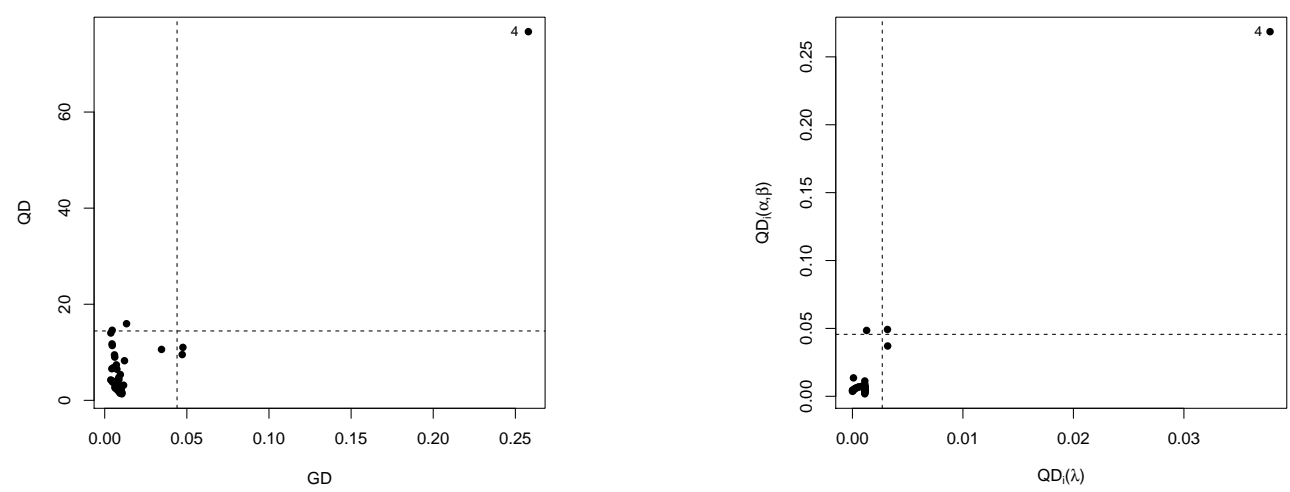

Figure 4.3: Biaxial dataset. Generalized Cook distance vs $Q$-distance (left) and $G D_{j}^{1}(\lambda)$ vs $G D_{j}^{1}(\alpha, \beta)$ (right).
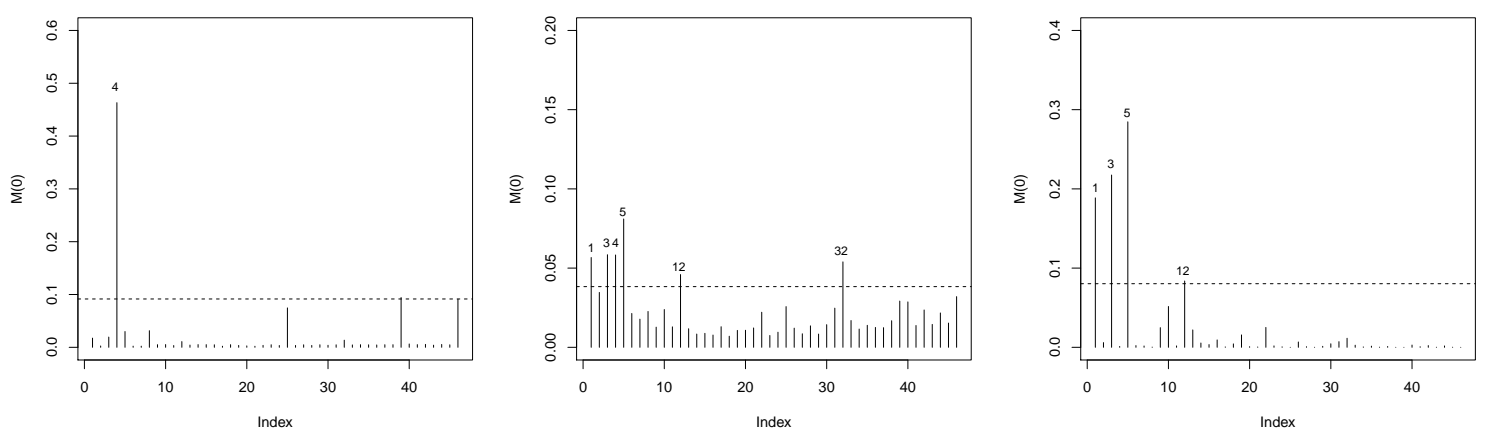

Figure 4.4: Biaxial dataset. Diagnostics of case weight perturbation (left), response variable perturbation (center) and work variable perturbation (right) (benchmark with $c^{*}=1$ ).

We add more information based on total local influence measures $C_{i}$, defined in (4.21), under the response and explanatory perturbations. Figure 4.5 shows the scatter plots of $C_{i}(\beta)$ versus $C_{i}(\alpha), C_{i}(\alpha)$ versus $C_{i}(\lambda)$ and $C_{i}(\beta)$ versus $C_{i}(\lambda)$. Figure $4.5(a)$ (response perturbation) shows that observations $\# 3, \# 4, \# 5$ and $\# 32$ have relatively large values of $C_{i}(\alpha)$ and $C_{i}(\beta)$. In turn, Figure 4.5(b)-(c) show that observation \#4 has relatively large values of $C_{i}(\alpha), C_{i}(\beta)$ and $C_{i}(\lambda)$, 
so observation \#4 is highly influential on the estimates of the both parameters $\beta$ and $\lambda$ under response perturbation. On the other hand, from Figure 4.5 (d)-(f) observations \#1, \#3 and \#5 have relatively large values of $C_{i}(\alpha), C_{i}(\beta)$ and $C_{i}(\lambda)$ and observation \#4 is not influential under explanatory perturbation.

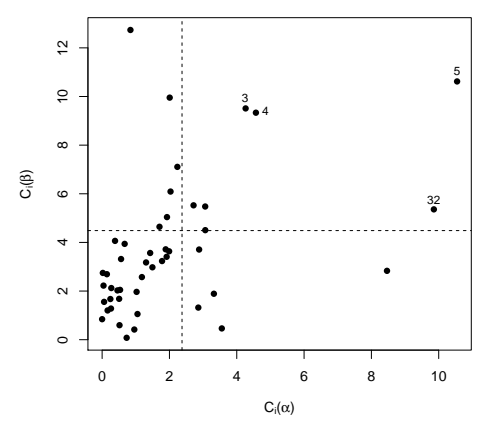

(a)

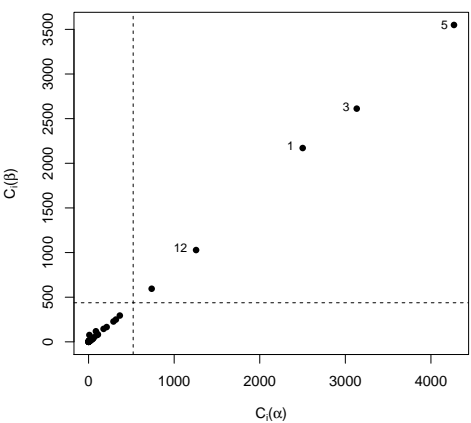

(d)

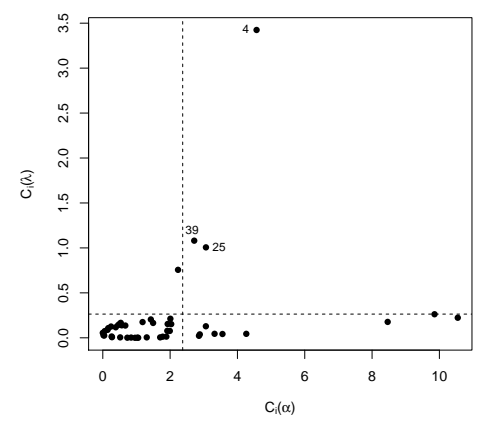

(b)

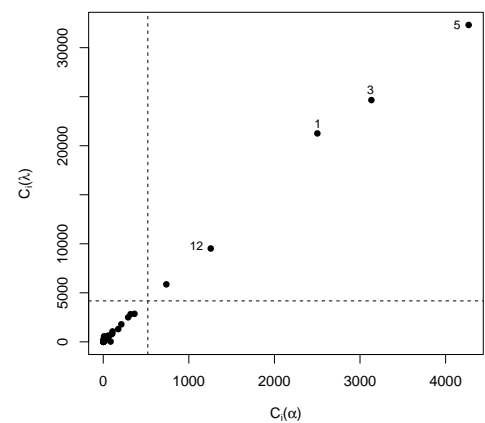

(e)

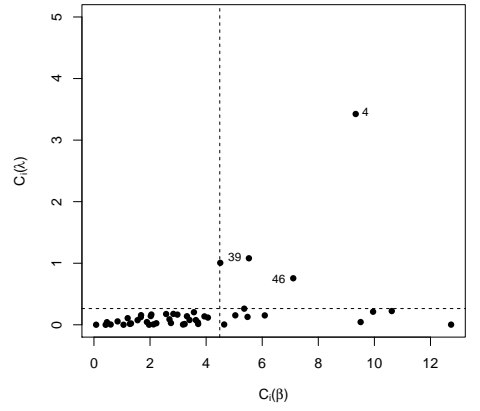

(c)

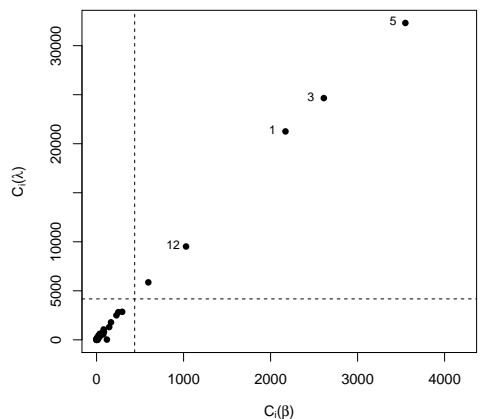

(f)

Figure 4.5: Biaxial dataset: $C_{i}$ for response perturbation (first line, $(a)-(c)$ ). $C_{i}$ for explanatory perturbation (second line, $(d)-(f))$.

Table 4.2 provides the relative changes of the ML estimates of the parameters (RC1) and relative changes of their asymptotic standard deviation estimates (RC2) considering model (4.23). These relative changes are defined as follows: $\mathrm{RC} 1=\left(\widehat{\theta}_{\mathrm{j}}-\widehat{\theta}_{\mathrm{j}(\mathrm{i})}\right) / \widehat{\theta}_{\mathrm{j}}$ and $\mathrm{RC} 2=\left(\widehat{\sigma}_{\widehat{\theta}_{\mathrm{j}}}-\widehat{\sigma}_{\widehat{\theta}_{\mathrm{j}(\mathrm{i})}}\right) / \widehat{\sigma}_{\widehat{\theta}_{\mathrm{j}}}$, where $\widehat{\theta}_{j}$ and $\widehat{\sigma}_{\widehat{\theta}_{j}}$ denote the ML estimates for $\theta_{j}$ and $\sigma_{\widehat{\theta}_{j}}$, respectively, after removing the $i$ th observation. Note that $\mathrm{RC} 2$ can also be interpreted as the relative change of the confidence interval amplitude by removing the $i$ th observation.

The results are grouped in Table 4.2, which shows no large changes for all parameters and confidence interval amplitudes. Therefore, we conclude that model (4.23) has little influence of such observations and is appropriate for this dataset (biaxial fatigue data). 
Table 4.2: Biaxial dataset: Relative changes (\%) after dropping the cases indicated.

\begin{tabular}{c|cc|cc|cc|cc}
\hline \hline \multirow{2}{*}{ Eliminated } & \multicolumn{2}{|c|}{$\alpha$} & \multicolumn{2}{|c|}{$\beta_{1}$} & \multicolumn{2}{c}{$\beta_{2}$} & \multicolumn{2}{|c}{$\lambda$} \\
& $\mathrm{RC} 1$ & $\mathrm{RC} 2$ & $\mathrm{RC} 1$ & $\mathrm{RC} 2$ & $\mathrm{RC} 1$ & $\mathrm{RC} 2$ & $\mathrm{RC} 1$ & $\mathrm{RC} 2$ \\
\hline 1 & 0.0048 & 0.0350 & -0.0074 & 0.0426 & -0.0079 & 0.0572 & -0.0152 & -0.1068 \\
3 & 0.0245 & 0.0563 & -0.0078 & 0.0789 & -0.0091 & 0.0881 & 0.0284 & -0.0021 \\
4 & 0.0083 & 0.2280 & 0.0472 & 0.0841 & 0.0552 & -0.0348 & -1.4795 & -4.9370 \\
5 & 0.0492 & 0.0197 & -0.0088 & 0.1197 & -0.0112 & 0.1218 & 0.0823 & 0.0988 \\
12 & 0.0377 & 0.0245 & -0.0026 & 0.0564 & -0.0035 & 0.0624 & 0.0540 & 0.0684 \\
32 & 0.0376 & -0.0266 & 0.0047 & -0.0552 & 0.0049 & -0.0543 & 0.0561 & 0.0783 \\
\hline \hline
\end{tabular}

\section{Martin Marietta dataset}

We here consider the Martin Marietta dataset analyzed by Butler et al. (1990) and Ferreira et al. (2015). This data set was also studied by Azzalini and Capitanio (2003) in asymmetrical models. On the basis of arguments presented by them, we propose the linear regression:

$$
Y_{i}=\beta_{0}+\beta_{1} \operatorname{CRSP}_{i} \varepsilon_{i} \quad i=1, \ldots, 60
$$

where the response $Y$ is the excess rate of return of the Martin Marietta company and CRSP is an index of the excess rate of return for the New York Stock Exchange as a whole. We assume that error terms are taken to be distributed as $\varepsilon_{i} \sim \operatorname{Sinh}-\operatorname{SNI}(\alpha, 0, \sigma=2 ; H)$. Data over a period of $n=60$ consecutive months are available.

Table 4.3 displays the ML estimates of the model parameters under the sinh-SN, sinh-ST, sinhSCN and sinh-SSL models and their corresponding standard errors (SE). For model comparison, we compute the $\ell(\boldsymbol{\theta})$ values, and the AIC, BIC and EDC (sinh-NI model, in parentheses). The criteria values indicate that the regression model in sinh-SNI models with heavy tails has a significantly better fit than the symmetric sinh-NI models. We note that the fit under the sinh-SCN and sinh-SSL models are significantly better than sinh-SN model. 
Table 4.3: Martin Marietta dataset: Results from fitting with four selected sinh-SNI models (information criteria values in sinh-NI model in parentheses).

\begin{tabular}{c|cc|cc|cc|cc}
\hline \hline \multirow{2}{*}{ Parameter } & \multicolumn{2}{|c|}{ Sinh-SN } & \multicolumn{2}{c|}{ Sinh-ST } & \multicolumn{2}{c|}{ Sinh-SCN } & \multicolumn{2}{c}{ Sinh-SSL } \\
& Estimate & SE & Estimate & SE & Estimate & SE & Estimate & SE \\
\hline$\alpha$ & 0.1373 & 0.0132 & 0.0625 & 0.0138 & 0.0670 & 0.0178 & 0.0493 & 0.0121 \\
$\beta_{0}$ & -0.0937 & 0.0143 & -0.0472 & 0.0210 & -0.0484 & 0.0264 & -0.0476 & 0.0237 \\
$\beta_{1}$ & 1.3891 & 0.3839 & 1.2455 & 0.2138 & 1.2665 & 0.2184 & 1.2631 & 0.2182 \\
$\lambda$ & 3.9496 & 2.0794 & 1.0841 & 0.8518 & 1.0594 & 0.9849 & 1.0717 & 0.9193 \\
$\nu$ & - & - & 3.1819 & - & 0.0836 & - & 1.1903 & - \\
$\gamma$ & - & - & - & - & 0.0526 & - & - & - \\
\hline$\ell(\widehat{\boldsymbol{\theta}})$ & $65.7676(57.2889)$ & $73.0699(71.8111)$ & $74.0996(72.9468)$ & $73.6296(72.4625)$ \\
$\mathrm{AIC}$ & $-123.5353(-108.5779)$ & $-138.1398(-137.6221)$ & $-140.1992(-139.8936)$ & $-139.2592(-138.925)$ \\
$\mathrm{BIC}$ & $-115.1579(-102.2948)$ & $-129.7625(-131.3391)$ & $-131.8218(-133.6106)$ & $-130.8818(-132.642)$ \\
EDC & $-125.3385(-109.9303)$ & $-139.9431(-138.9746)$ & $-142.0024(-141.2461)$ & $-141.0624(-140.2775)$ \\
\hline \hline
\end{tabular}

In order to detect outlying observations, first we use the Mahalanobis distance. Figure 4.6 displays the boxplots for these distances for the four fitted models. It can be seen from these figures that observation \#8 appears to be an outlier. Case-deletion measures based on Cook's
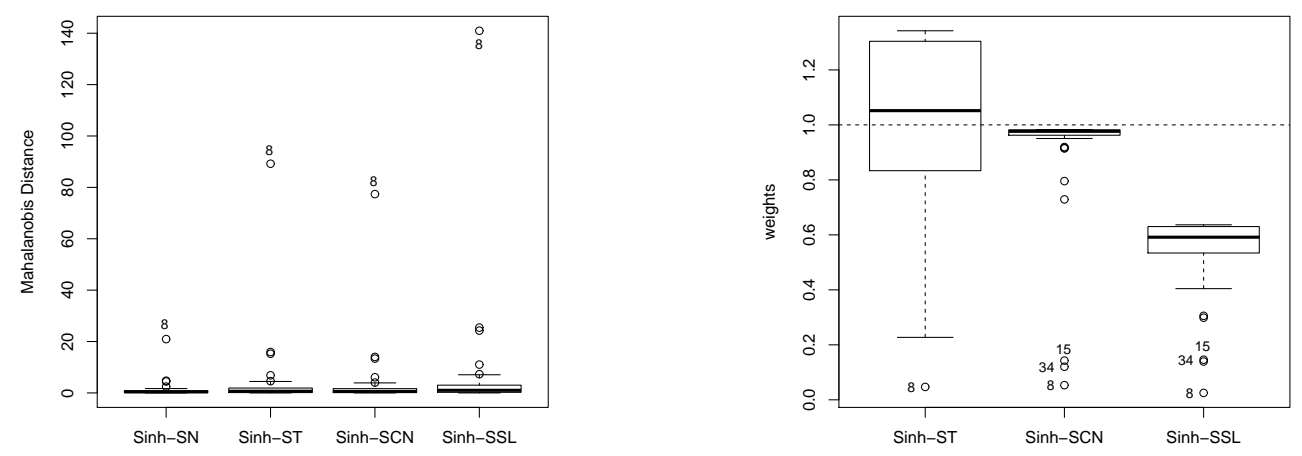

Figure 4.6: Martin Marietta dataset: Boxplots of the Mahalanobis distances for the four fitted models (left) and the estimated with $\kappa_{i}$ in the sinh-N, sinh-ST, sinh-SCN and sinh-SSL models (right).

distance and $Q$-distance are displayed in Figure 4.7. Considering the generalized Cook's distance and $Q$-distance, we observe that cases \#8 and \#58 are identified as the most influential in the estimation of the parameters under the sinh-SN model. Both of these cases at the left of the figure. Meanwhile, no observations are influential in the sinh-ST, sinh-SCN and sinh-SSL cases, of which only the sinh-ST case is shown in Figure 4.7 (right). The same pattern can been seen when we use the Q-distance, where only observations \#8 and \#58 are influential in the sinh-SN. For this dataset, the sinh-ST, sinh-SCN and sinh-SSL better accommodate the influential observations. As 
expected, the influence of such observations is reduced when distributions with heavier tails than the sinh-SN one are considered.
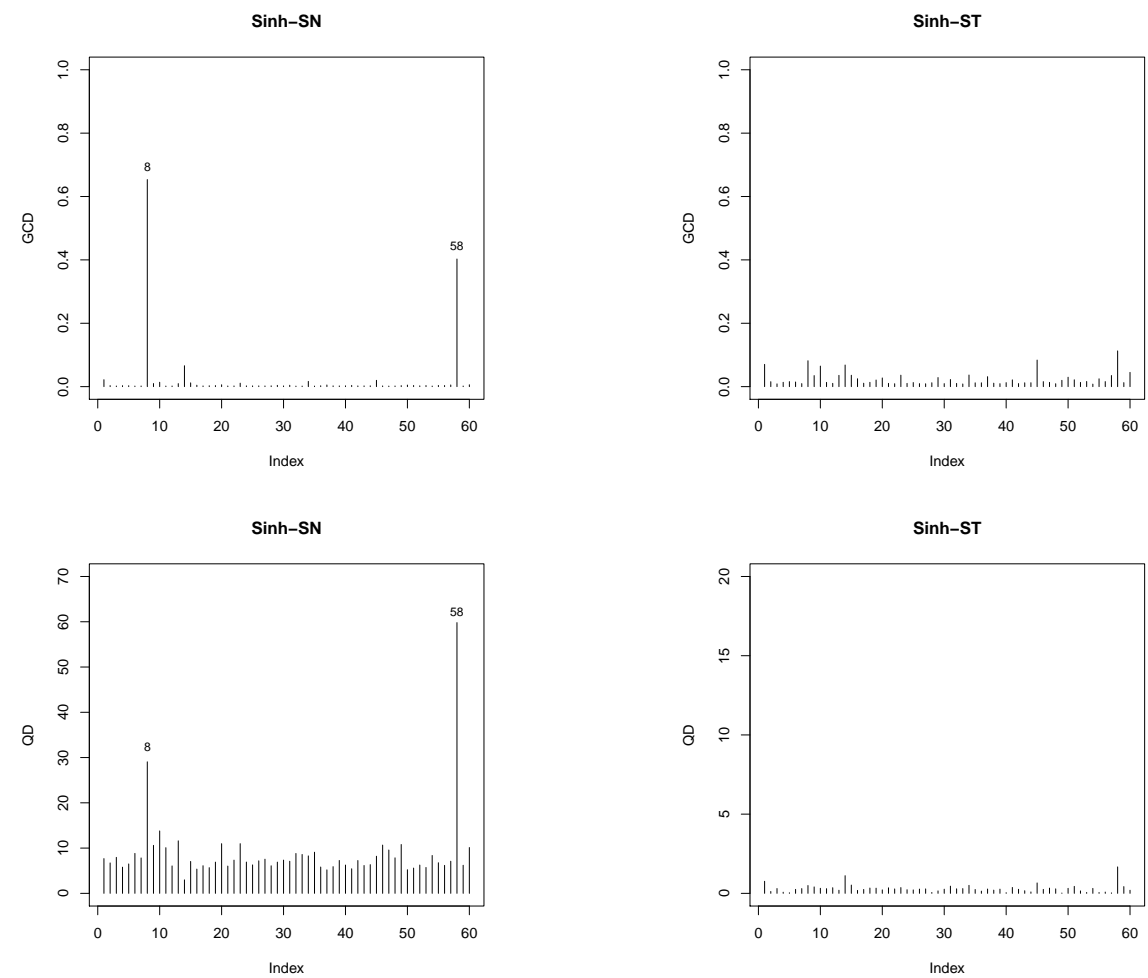

Figure 4.7: Martin Marietta dataset: Index plot of generalized Cook distance (first line) and index plot of $Q$-distance (second line) using sinh-SN and sinh-ST models.

Finally, we identify influential observations for the Martin Marietta data set by using $M(0)$ from the conformal curvature $B_{f_{Q}, \mathbf{u}_{l}}$ obtained by considering the case-weights perturbation, the response and the CRSP variable perturbation schemes. Figures 4.8 present the index graphs of $M(0)$ for the four selected models.

From Figure 4.8, case \#8 seems to be the most influential in the ML estimators in the sinhSN model under all perturbation schemes. However, when using sinh-ST, sinh-SCN and sinh-SSL models, there are no influential observations. From these results, we only report the sinh-ST case at the right of the figure. 

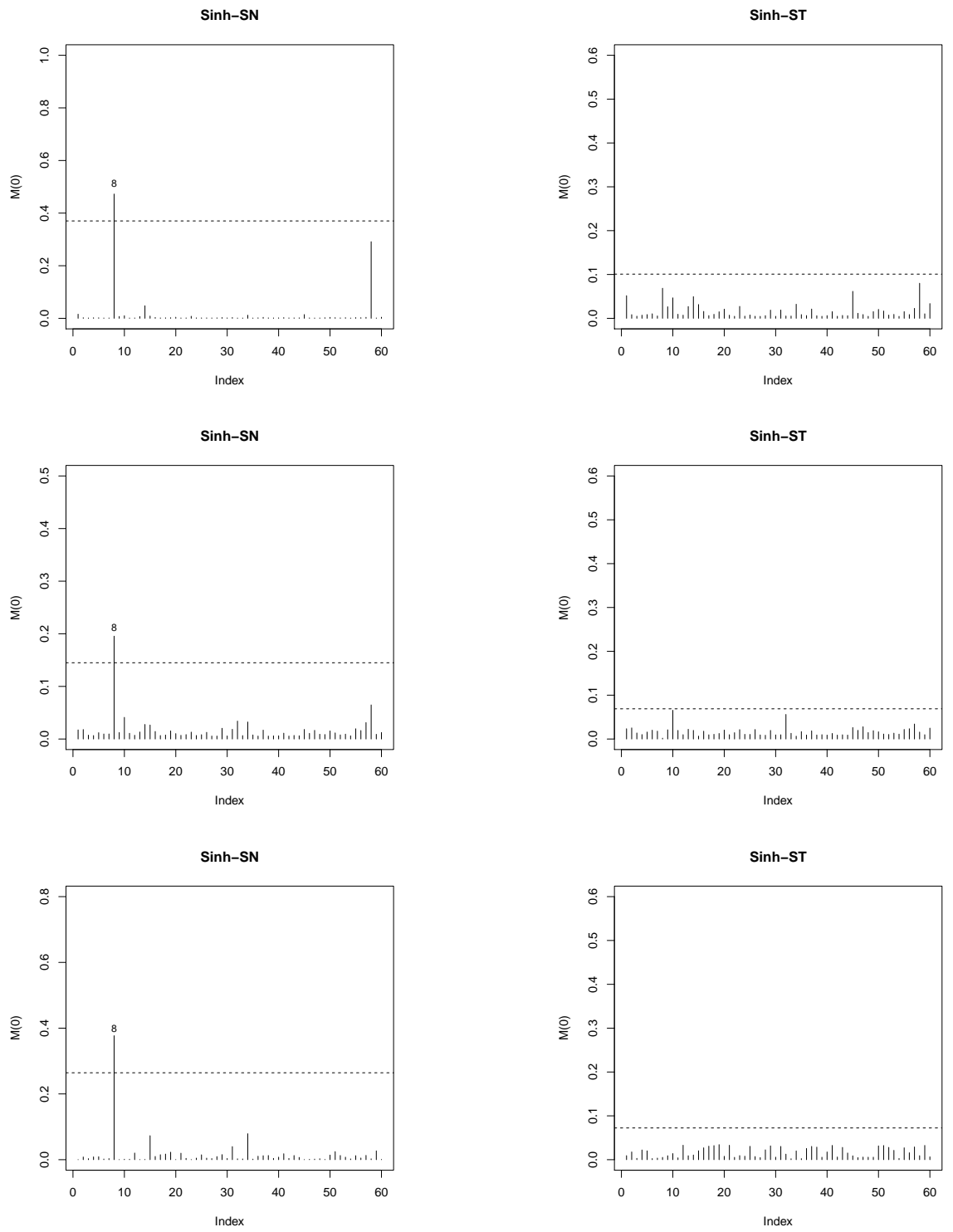

Figure 4.8: Martin Marietta dataset: Index plot of $M(0)$ in the case weights perturbation (first line), response perturbation (second line) and explanatory perturbation (third line) in the sinh-SN and sinh-ST models.

Also, to reveal the impact of the detected influential observations \#8 and \#58, we use the quantities TRC (total relative changes) and MRC (maximum relative changes), suggested by Lu and Song (2006), which are defined as TRC $=\sum_{j=1}^{n_{p}}\left|\left(\widehat{\theta}_{j}-\widehat{\theta}_{[i] j}\right) / \widehat{\theta}_{j}\right| \quad$ and $\quad \mathrm{MRC}=\max _{j=1, \ldots, n_{p}} \mid\left(\widehat{\theta}_{j}-\right.$ $\left.\widehat{\theta}_{[i] j}\right) / \widehat{\theta}_{j} \mid$, respectively, where $n_{p}$ is the dimension of $\boldsymbol{\theta}$ and the subscript $[i]$ means the ML estimator of $\boldsymbol{\theta}$ with the $i$ th observation is deleted. The comparison results of these measures, with the observations \#8 and \#58 deleted, are given in Table 4.4, where larger changes occur with the sinh-SN distribution. As expected, the results show that the ML estimates are less sensitive to the presence of atypical data when distributions with heavier tails than the sinh-SN are used. Thus, our main conclusion from this example is that the ML estimates from the sinh-SNI models are more robust against outlying observations than the sinh-SN model under all perturbation schemes considered. 
Table 4.4: Martin Marietta dataset: Comparison of the relative changes in the ML estimates in terms of $T R C$ and MRC for the four selected sinh-SNI models.

\begin{tabular}{|c|cc|cc|cc|}
\hline \hline & \multicolumn{2}{|c|}{8} & \multicolumn{2}{c|}{58} & \multicolumn{2}{c|}{8 and 58} \\
& TRC & MRC & TRC & MRC & TRC & MRC \\
\hline Sinh-SN & 5.1257 & 3.6800 & 2.2039 & 2.1397 & 3.2598 & 1.8512 \\
Sinh-ST & 0.1909 & 0.1004 & 0.8542 & 0.5145 & 1.4405 & 0.8138 \\
Sinh-SCN & 0.6025 & 0.2718 & 0.7938 & 0.5074 & 0.2046 & 0.0951 \\
Sinh-SSL & 0.1952 & 0.1170 & 0.9106 & 0.5550 & 1.2491 & 0.6690 \\
\hline \hline
\end{tabular}

\subsection{Conclusions}

In this paper, we have provided a diagnostics analysis for sinh-SNI regression models, which contain a generalization of the log-BS regression models proposed by Rieck and Nedelman (1991). The diagnostic procedures are based on those proposed by Zhu et al. (2001) and Zhu and Lee (2001), since to use the observed-data log-likelihood function for the proposed regression model is rather complicated to detect influential observations by direct application of the traditional approaches given in Cook $(1977,1986)$.

We have proposed a procedure for computing case-deletion measures and local influence diagnostics on the basis of the conditional expectation of the complete-data log-likelihood function in relation to the EM algorithm. Case weight perturbation, response and explanatory perturbations are considered. Explicit expressions are obtained for the Hessian matrix $\ddot{Q}(\widehat{\boldsymbol{\theta}} \mid \widehat{\boldsymbol{\theta}})$ and for the matrix $\boldsymbol{\Delta} \boldsymbol{\omega}_{0}$ under all different perturbation schemes. The case-deletion measures help to detect individual observations, so it can be important to have a general idea on outlying observations. However, the diagnostic measures on the basis of the local influence approach involving perturbation schemes help to identify the reasons why the observations are influential. 
84 INFLUENCE ANALYSIS IN SINH-SKEW-NORMAL/INDEPENDENT REGRESSION MODELS 


\section{Chapter 5}

\section{Conclusions}

\subsection{Final considerations}

In this thesis, a study of estimation and diagnostics was developed in the new class of BS distributions based on the scale mixture of skew-normal and regression models, in turn based on the sinh-skew-normal/independent distributions family. The diagnostic analysis was based on the local influence method, which was proposed by Zhu and Lee (2001) for incomplete data. Our results generalize some results proposed by Birnbaum and Saunders (1969a), Vilca et al. (2011), Balakrishnan et al. (2009), Rieck and Nedelman (1991) and Vilca et al. (2015), among others.

The SMSN-BS distributions have as special cases the distributions based on the skew-normal, skew$t$, skew-slash and skew-contaminated normal models, among others. This new class of distributions is more robust, since allows us to predict extreme percentiles, especially in the left tail, which can not be easily estimated using BS models based on symmetric distributions. Furthermore, its hierarchical representation facilitates the implementation of the EM algorithm and also allows obtaining the observed information matrix.

Some special cases of the sinh-skew-normal/independent family of distributions are the asymmetric sinh-SN, sinh-ST, sinh-SCN and sinh-SSL, an important case being the log-Birnbaum-Saunders distribution used in the context of linear regression. In this thesis we propose a Sinh-SNI nonlinear regression model which allows generalizing both linear and nonlinear cases. In addition, we present an EM algorithm to obtain the maximum likelihood, which allows obtaining robust estimates in the presence of atypical observations, making it a very flexible distribution for modeling purposes.

In this work, a procedure based on the conditional expectation of the complete log-likelihood 
function related to the EM algorithm was used to compute the case-deletion measures of the local influence, which helps to detect outlying observations. On the other hand, the local influence approach involving perturbation schemes is useful in finding the reasons why observations are influential.

\subsection{Suggestions for future research}

Several research works can be derived and/or directed from the results of this work, among them we can mention the following:

- We are developing score tests of heteroscedasticity and/or autocorrelation in nonlinear models with AR(1) and Sinh-Normal erros. The resulting model provided an useful slight extension of the log-BS regression models in which the variance depends of the shape parameter of the BS distribution. This proposal is useful because in regression models it is usually assumed that random erros are independent each other. However, according to Bates and Watts (1988) the situations in which data are collected sequentially over the time may raise substantial autocorrelations in the erros. Also it is usually assumed that the variance of white noises are homogeneous. However, in some situations, these assumptions are not necessarily appropriate. The asymptotic properties of the score statistic, including chi-square and approximate powers under local alternatives, are being studied and examined by Monte Carlo simulations. We are looking for a real dataset appropriate to illustrate our test methods.

- To study the results of estimation and diagnostics of the SMSN-BS distribution class and regression models based on the sinh-SNI distribution family from a Bayesian perspective.

- To extend the estimation and diagnostic results of the SMSN-BS and sinh-SNI-RM models to the multivariate case; and

- To study the estimation and diagnostic results considering censored data in the SMSN-BS and sinh-SNI-RM models. 


\section{Appendix A}

\section{Special cases of sinh-SNI models}

\section{i) The sinh-SCN distribution}

This distribution is based on the SCN model, which is called sinh-skew-contaminated normal (sinh-SCN) distribution. Here, $U$ is a discrete random variable with pdf $h(u ; \nu, \gamma)=\nu \mathbb{I}_{\{\gamma\}}(u)+$ $(1-\nu) \mathbb{I}_{\{1\}}(u)$, with $0<\nu<1,0<\gamma<1$, where $\mathbb{I}_{A}(\cdot)$ denotes the indicator function of the set $A$. Then, the pdf of $Y$ is

$$
f_{Y}(y)=\frac{2}{\sigma}\left[\nu \phi\left(\xi_{2 y} ; 0, \frac{1}{\gamma}\right) \Phi\left(\gamma^{1 / 2} \lambda \xi_{2 y}\right)+(1-\nu) \phi\left(\xi_{2 y}\right) \Phi\left(\lambda \xi_{2 y}\right)\right] \xi_{1 y}
$$

This distribution is denoted by $Y \sim \operatorname{Sinh}-\operatorname{SNC}(\alpha, \mu, \lambda ; \nu, \gamma)$. In this case,

$$
\begin{gathered}
\kappa_{r}=\frac{2 \xi_{1 y}}{\sigma f_{Y}(y)}\left[\nu \gamma^{r} \phi\left(\xi_{2 y} ; 0, \gamma^{-1}\right) \Phi\left(\gamma^{1 / 2} \lambda \xi_{2 y}\right)+(1-\nu) \phi\left(\xi_{2 y}\right) \Phi\left(\lambda \xi_{2 y}\right)\right] \\
\tau_{r}=\frac{2 \xi_{1 y}}{\sigma f_{Y}(y)}\left[\nu \gamma^{r / 2} \phi\left(\xi_{2 y} ; 0, \gamma^{-1}\right) \Phi\left(\gamma^{1 / 2} \lambda \xi_{2 y}\right)+(1-\nu) \phi\left(\xi_{2 y}\right) \Phi\left(\lambda \xi_{2 y}\right)\right] .
\end{gathered}
$$

Finally, $d(Y)$ has its cdf as $\operatorname{Pr}(d(Y) \leq v)=\nu \operatorname{Pr}\left(\chi_{1}^{2} \leq \gamma v\right)+(1-\nu) \operatorname{Pr}\left(\chi_{1}^{2} \leq v\right)$;

ii) The sinh-SSL distribution

In this case, $U \sim \operatorname{Beta}(\nu, 1)$ distribution, and the resulting distribution is called the sinhskew-slash ( $\sinh -\mathrm{SSL})$ distribution, denoted by $Y \sim \operatorname{Sinh}-\operatorname{SSL}(\alpha, \mu, \lambda ; \nu)$, and its pdf is given by

$$
f_{Y}(y)=\frac{2 \nu}{\sigma} \int_{0}^{1} u^{\nu-1} \phi\left(\xi_{2 y} ; 0,1 / u\right) \Phi\left(u^{1 / 2} \lambda \xi_{2 y}\right) d u \xi_{1 y}
$$

In this case,

$$
\kappa_{r}=\frac{2^{\nu+r+1} \nu \Gamma\left(\frac{2 \nu+2 r+1}{2}\right) \xi_{1 y}}{f_{Y}(y) \sqrt{\pi} \sigma} P_{1}\left(\frac{2 \nu+2 r+1}{2}, \frac{\xi_{2 y}^{2}}{2}\right) \xi_{2 y}^{-(2 \nu+2 r+1)} \mathrm{E}\left[\Phi\left(S^{1 / 2} \lambda \xi_{2 y}\right)\right]
$$




$$
\tau_{r}=\frac{2^{\nu+r / 2+1 / 2} \nu \Gamma\left(\frac{2 \nu+r+1}{2}\right) \xi_{1 y}}{f_{Y}(y) \sqrt{\pi}^{2} \sigma}\left(\xi_{2 y}^{2}+\lambda^{2} \xi_{2 y}^{2}\right)^{-\frac{2 \nu+r+1}{2}} P_{1}\left(\frac{2 \nu+r+1}{2}, \frac{\xi_{2 y}^{2}+\lambda^{2} \xi_{2 y}^{2}}{2}\right),
$$

where $P_{x}(a, b)$ denotes the cdf of the $\operatorname{Gamma}(a, b)$ distribution evaluated at $x$ and $S \sim$ Gamma $\left(\frac{2 \nu+2 r+1}{2}, \frac{\xi_{2 y}^{2}}{2}\right) \mathbb{I}_{(0,1)}$, a truncated gamma distribution on $(0,1)$. Finally, the cdf of $d(Y)$ is $\operatorname{Pr}(d(Y) \leq v)=\operatorname{Pr}\left(\chi_{1}^{2} \leq v\right)-\frac{2^{\nu} \Gamma(\nu+1 / 2)}{v^{\nu} \Gamma(1 / 2)} \operatorname{Pr}\left(\chi_{2 \nu+1}^{2} \leq v\right) ;$

iii) The sinh-ST distribution

This distribution is obtained when $U \sim \operatorname{Gamma}(\nu / 2, \nu / 2)$, and the resulting distribution is called the sinh-skew-Student- $t$ (sinh-ST) distribution. The pdf of $Y$ is

$$
f_{Y}(y)=\frac{2 t}{\sigma}\left(\xi_{2 y} ; \nu\right) T\left(\sqrt{\frac{\nu+1}{\xi_{2 y}^{2}+\nu}} \lambda \xi_{2 y} ; \nu+1\right) \xi_{1 y}
$$

where $t(. ; \nu)$ and $T(. ; \nu)$ denote, respectively, the pdf and cdf of the standard Student- $t$ distribution. In this case, the conditional expectations are given by

$$
\kappa_{r}=\frac{2^{r+1} \nu^{\nu / 2} \Gamma\left(\frac{\nu+2 r+1}{2}\right)\left(\xi_{2 y}^{2}+\nu\right)^{-\frac{\nu+2 r+1}{2}} \xi_{1 y}}{f_{Y}(y) \Gamma(\nu / 2) \sqrt{(\pi)} \sigma} T\left(\sqrt{\frac{\nu+2 r+1}{\xi_{2 y}^{2}+\nu}} \lambda \xi_{2 y} ; \nu+2 r+1\right)
$$

and

$$
\tau_{r}=\frac{2^{(r+1) / 2} \nu^{\nu / 2} \Gamma\left(\frac{\nu+r+1}{2}\right)\left(\xi_{2 y}^{2}+\nu+\lambda^{2} \xi_{2 y}^{2}\right)^{-\frac{\nu+r+1}{2}} \xi_{1 y}}{f_{Y}(y) \Gamma(\nu / 2) \sqrt{(\pi)}^{2} \sigma} .
$$

Finally, we have $d(Y) \sim F(1, \nu)$. 


\section{Bibliography}

Achcar(1993) J. A. Achcar. Inference for the Birnbaum-Saunders fatigue life model using Bayesian methods. Comp. Stat. Data Anal., 15:367-380. Cited in page 2

Andrews and Mallows(1974) D. F. Andrews and C. L. Mallows. Scale mixtures of normal distributions. J. Roy. Statist. Soc., Ser. B, 36:99-102. Cited in page 3, 5, 36, 62

Azzalini(1985) A. Azzalini. A class of distributions which includes the normal ones. Scand. J. Stat., 12:171-178. Cited in page 4, 5, 13, 14, 36, 62

Azzalini and Capitanio(2003) A. Azzalini and A. Capitanio. Distributions generated by perturbation of symmetry with emphasis on a multivariate skew-t distribution. J. Roy. Statist. Soc., Ser. B, 65:367-389. Cited in page 55, 75, 79

Balakrishnan et al.(2009) N. Balakrishnan, V. Leiva, A. Sanhueza and F. Vilca. Estimation in the Birnbaum-Saunders distribution based on scale-mixture of normals and the EMalgorithm. Stat. Oper. Res. T., 33:171-192. Cited in page 2, 4, 12, 15, 18, 24, 33, 37, 38, $41,59,63,85$

Barros et al.(2008) M. Barros, G. A. Paula and V. Leiva. A new class of survival regression models with heavy-tailed errors: robustness and diagnostics. Lifetime Data Analysis, 14:316-332. Cited in page 2, 12

Barros et al.(2009) M. Barros, G. A. Paula and V. Leiva. An R implementation for generalized Birnbaum-Saunders distributions. Comp. Stat. Data Anal., 53:1511-1528. Cited in page 2

Basford et al.(1997) K. E. Basford, D. R. Greenway, G. J. McLachlan and D. Peel. Standard errors of fitted component means of normal mixtures. Comput. Stat., 12:1-17. Cited in page 25,46

Basso et al.(2009) R. M. Basso, V. H. Lachos, C. R. Cabral and P. Ghosh. Robust mixture modeling based on scale mixtures of skew-normal distributions. Comp. Stat. Data Anal., 54:2926-2941. Cited in page 25

Bates and Watts(1988) D. Bates and D. G. Watts. Nonlinear regression analysis and its applications. Wiley, New York. Cited in page 86

Birnbaum and Saunders(1969a) Z. W. Birnbaum and S. C. Saunders. A new family of life distributions. J. Appl. Prob., 6:637-652. Cited in page iii, v, 1, 2, 3, 12, 33, 36, 62, 85

Birnbaum and Saunders(1969b) Z. W. Birnbaum and S. C. Saunders. Estimation for a family of life distributions with applications to fatigue. J. Appl. Prob., 6:328-347. Cited in page iii, v, 1,62

Branco and Dey(2001) M. D. Branco and D. K. Dey. A general class of multivariate skewelliptical distributions. J. Multivar. Anal., 79:99-113. Cited in page 4, 14, 36 
Butler et al.(1990) R. L. Butler, J. B. McDonald, R. D. Nelson and S. B. White. Robust and partly adaptive estimation of regression models. Rev. Econ. Statist., 72:321-327. Cited in page $55,75,79$

Chatterjee and Hadi(1988) S. Chatterjee and A. S. Hadi. Sensitivity Analysis in Linear Regression. Wiley, New York. Cited in page 7

Cook and Weisberg(1982) R. Cook and S. Weisberg. Residuals and Influence in Regression. Chapman \& Hall, Boca Raton, FL. Cited in page 7

Cook(1977) R. D. Cook. Detection of influential observations in linear regression. Technometrics, 19:15-18. Cited in page $61,63,67,69,83$

Cook(1986) R. D. Cook. Assessment of local influence (with discussion). J. R. Stat. Soc. B, 48: 133-169. Cited in page $2,4,7,8,61,63,67,70,83$

Cordeiro et al.(2013) G. M. Cordeiro, A. J. Lemonte and E. M. M. Ortega. An extended fatigue life distribution. Statistics: A Journal of Theoretical and Applied Statistics, 47:3:626653. Cited in page 9,29

Dempster et al.(1977) A. P. Dempster, N. M. Laird and D. B. Rubin. Maximum likelihood from incomplete data via the EM algorithm. J. Roy. Statist. Soc., Ser. B, 39:1-38. Cited in page $37,43,65$

Dempster et al.(1980) A. P. Dempster, N. M. Lair and D. B. Rubin. Iteratively reweighted least squares for linear regression when errors are Normal/Independent distributed. editor (Krishnaiah P. R.), North-Holland, Amsterdam, multivariate analysis, volume v edição. Cited in page 5

Desmond(1985) A. F. Desmond. Stochastic models of failure in random environments. Canadian Journal of Statistics, 13:171-183. Cited in page 1, 12

Desmond(1986) A. F. Desmond. On the relationship between two fatigue-life models. IEEE Transactions on Reliability, 35:167-169. Cited in page 2

Díaz-García and Leiva(2005) J.A. Díaz-García and V. Leiva. A new family of life distributions based on elliptically contoured distributions. Journal of Statistical Planning and Inference, 137:1512-1513. Cited in page 2, 3, 12

Dupuis and Mills(1998) D. J. Dupuis and J. E. Mills. Robust estimation of the BirnbaumSaunders distribution. IEEE Transactions on Reliability, 47:88-95. Cited in page 2

Engelhardt et al.(1981) M. Engelhardt, L. J. Bain and F. T. Wright. Inferences on the parameters of the Birnbaum-Saunders fatigue life distribution based on maximum likelihood estimation. Technometrics, 23:251-256. Cited in page 1

Farias and Lemonte(2011) R. B. A. Farias and A. J. Lemonte. Bayesian inference for the Birnbaum-Saunders nonlinear regression model. Stat. Methods Appl., 20:423-438. Cited in page 53

Ferreira et al.(2015) C. S. Ferreira, V. H. Lachos and H. Bolfarine. Inference and diagnostics in skew scale mixtures of normal regression models. J. Stat. Comput. Simul., 85(3): 517-537. Cited in page 55, 56, 75, 79

Galea et al.(1997) M. Galea, G. A. Paula and H. Bolfarine. Local influence in elliptical linear regression models. The Statistician, 46:71-79. Cited in page 7

Galea et al.(2004) M. Galea, V. Leiva and G. A. Paula. Influence diagnostics in log-BirnbaumSaunders regression models. J. Appl. Stat., 31:1049-1064. Cited in page 2 
Galea et al.(2005) M. Galea, H Bolfarine and F. Vilca-Labra. Local influence in comparative Calibration models under elliptical t-distribution. Biometrical Journal, 5:691-706. Cited in page 7

Genton(2004) M. G. Genton. Skew-elliptical Distributions and Their Applications: A Journey Beyond Normality. Chapman and Hall/CRC Press, Boca Raton, FL. Cited in page 12

Gomez et al.(2009) H. W. Gomez, J. Olivares and H. Bolfarine. An extension of the generalized Birnbaum-Saunders distribution. Statistical and Probability Letters, 79:331-338. Cited in page 12

Gupta(2003) A. K. Gupta. Multivariate skew t-distributions. Statistics, 37(4):359-363. Cited in page 6

Gupta and Gupta(2008) R. C. Gupta and R. D. Gupta. Analyzing skewed data by power normal model. 17:197-210. Cited in page 18

Johnson(1949) N. L. Johnson. Systems of frequency curves generated by methods of translation. Biometrika, 36:146-176. Cited in page 35

Johnson et al.(1994) N. L. Johnson, S; Kotz and N. Balakrishnan. Continuous Univariate Distributions-Vol. 1. John Wiley \& Sons, New York, second edition edição. Cited in page 36

Kundu et al.(2010) D. Kundu, N. Balakrishnan and A. Jamalizadeh. Bivariate BirnbaumSaunders distribution and associated inference. Journal of Multivariate Analysis, 101: 113-125. Cited in page 13

Kundu et al.(2013) P. Kundu, N. D. Brenowitz, V. Voon, Y. Worbe, P. E. Vertes, S. J. Inati, Z. S. Saad, P. A. Bandettini and E. T. Bullmore. Integrated strategy for improving functional connectivity mapping using multiecho fMRI. Proc Natl Acad Sci U S A, 110: 16187-16192. Cited in page 45

Lachos et al.(2010a) V. H. Lachos, P. Ghosh and R. B. Arellano-Valle. Likelihood based inference for skew normal independent linear mixed models. Stat. Sin., 20:303-322. Cited in page $26,40,46$

Lachos et al.(2010b) V. H. Lachos, F. Vilca-Labra, H. Bolfarine and P. Ghosh. Multivariate Measurement Error Models Based on Scale Mixtures of the Skew Normal Distribution. Statistics, 44:541-556. Cited in page 6

Lange and Sinsheimer(1993) K. Lange and J. S. Sinsheimer. Normal/independent distributions and their applications in robust regression. J. Comp. Graph. Stat., 2:175-198. Cited in page $4,5,14,18,36,41,62,65$

Lange et al.(1989) K. L. Lange, J. A. Little and M. G. J. Taylor. Robust statistical modeling using the t distribution. J. Amer. Stat. Assoc., 84:881-896. Cited in page 6, 44, 65

Lee and $\mathrm{Xu}(\mathbf{2 0 0 4}) \mathrm{S}$. Y. Lee and $\mathrm{L}$. Xu. Influence analysis of nonlinear mixed-effects models. Comp. Stat. Data Anal., 45:321-341. Cited in page 63, 68, 71

Lee et al.(2006) S. Y. Lee, B. Lu and X. Y. Song. Assessing local influence for nonlinear structural equation models with ignorable missing data. Comp. Stat. Data Anal., 5:1356-1377. Cited in page 58

Leiva et al.(2007) V. Leiva, M. Barros, G. A. Paula and M. Galea. Influence diagnostics in log-Birnbaum-Saunders regression models with censored. Comp. Stat. Data Anal., 51: 5694-5707. Cited in page 12 
Leiva et al.(2008a) V. Leiva, M. Barros, G. Paula and D. Sanhueza. Generalized BirnbaumSaunders distributions applied to air pollutant concentration. Environmetrics, 19:235249. Cited in page 2,12

Leiva et al.(2008b) V. Leiva, M. Riquelme, N. Balakrishnan and A. Sanhueza. Lifetime analysis based on the generalized Birnbaum-Saunders distribution. Comp. Stat. Data Anal., 52: 2079-2097. Cited in page 12

Leiva et al.(2009) V. Leiva, A. Sanhueza and J. M. Angulo. A length-biased version of the Birnbaum-Saunders distribution with application in water quality. Stoch Environ Res Risk Assess, 23:299-307. Cited in page 2, 12

Leiva et al.(2010) V. Leiva, F. Vilca, N. Balakrishnan and A. Sanhueza. A skewed Sinh-Normal distribution and its properties and application to air pollution. Comm. Stat. Theor. Meth., 39:426-443. Cited in page 2, 12, 16, 36, 38, 39, 63

Lemonte and Cordeiro(2009) A. J. Lemonte and G. M. Cordeiro. Improved maximum likelihood estimation in Birnbaum-Saunders nonlinear regressions. Comp. Stat. Data Anal., 53: 4441-4452. Cited in page $36,37,42,43,54,59,62,64,74,75$

Lemonte and Patriota(2011) A. J. Lemonte and A. G. Patriota. Influence diagnostics in Birnbaum-Saunders nonlinear regression models. J. Appl. Stat., 38:871-884. Cited in page 75

Lesaffre and Verbeke(1998) E. Lesaffre and G. Verbeke. Local influence in Linear Mixed Moldels. Biometrics, 54:570-582. Cited in page 7

Lin(1994) D. Y. Lin. Cox regression analysis of multivariate failure time data: The marginal approach. Stat. Med., 13:2233-2247. Cited in page 53

Lin et al.(2007) T. I. Lin, J. C. Lee and S. Y. Yen. Finite mixture modelling using the skew normal distribution. Stat. Sin., 17:909-927. Cited in page 25, 46

Little(1988) R. J. A. Little. Robust estimation of the mean and covariance matrix from data with missing values. Applied Statistics, 37:23-38. Cited in page 6

Lu and Song(2006) B. Lu and X. Y. Song. Local influence of multivariate probit latent variable models. J. Multivar. Anal., 97:1783-1798. Cited in page 71, 82

Lu and Chang(1997) M. Lu and D. S. Chang. Bootstrap prediction intervals for the BirnbaumSaunders distribution. Microelectron Reliability:1213-1216. Cited in page 2

Maehara and Benites(2015) R. Maehara and L. Benites. bssn: Birnbaum-Saunders Model Based on Skew-Normal Distribution. $R$ package version 0.6. Cited in page 25

Maehara et al.(2017) R. P. Maehara, F. Vilca and H. Bolfarine. Modeling extreme percentiles in Birnbaum-Saunders distributions based on an asymmetric heavy-tailed distribution. Under review. Cited in page 38, 62, 63, 64, 65

Mann et al.(1974) N. R. Mann, R. E. Schafer and N. Singpurwalla. Methods for Statistical Analysis of Reliability and Life Data. John Wiley and Sons, New York. Cited in page 1

Marshall and Olkin(2007) A. W. Marshall and I. Olkin. Life Distributions. Springer, New York. Cited in page 2

Matos et al.(2013) L. A. Matos, V. H. Lachos, N. Balakrishnan and F. Vilca-Labra. Influence diagnostics in linear and nonlinear mixed-effects models with censored data. Comp. Stat. Data Anal., 57:450-464. Cited in page 4 
Ng et al.(2003) H.K. Ng, D. Kundu and N. Balakrisnan. Modified moment estimation for the two-parameter Birnbaum-Saunders distribution. Comp. Stat. Data Anal., 43:283-298. Cited in page 25

Nichols and Padgett(2006) M.D. Nichols and W.J. Padgett. A Bootstrap Control Chart for Weibull Percentiles. Qual. Reliab. Eng. Int., 22:141-151. Cited in page 9, 29

O'Hagan and Leonard(1976) A. O'Hagan and T. Leonard. Bayes estimation subject to uncertainty about constraints. Biometrika, 63:201-203. Cited in page 4

Ortega et al.(2003) E. M. Ortega, H. Bolfarine and G. A. Paula. Influence diagnostics in generalized log-gamma regression models. Comp. Stat. Data Anal., 42:165-186. Cited in page 73

Owen and Padgett(2000) W. J. Owen and W. J. Padgett. A Birnbaum-Saunders accelerated life model. IEEE Transactions on Reliability, 49:224-229. Cited in page 2, 12

Paula et al.(2011) G. A. Paula, V. Leiva, M. Barros and S. Liu. Robust statistical modeling using the Birnbaum-Saunders-t distribution applied to insurance. Appl. Stoc. Models Bus. Ind., 28:16-34. Cited in page 36, 43, 59, 64

Podlaski(2008) R. Podlaski. Characterization of diameter data in near-natural forests using the Birnbaum-Saunders distribution. Can. J. For. Res., 38:518-527. Cited in page 12

Rieck(1989) J. R. Rieck. Statistical Analysis for the Birnbaum-Saunders Fatigue Life Distribution. Tese de Doutorado, Clemson University, South Carolina. Cited in page 2, 35, 36, 37, 62

Rieck and Nedelman(1991) J. R. Rieck and J. R. Nedelman. A log-linear model for the Birnbaum-Saunders distribution. Technometrics, 33:51-60. Cited in page iii, v, 2, 35, 36, $42,53,59,61,74,85$

Santana et al.(2011) L. Santana, F. Vilca and V. Leiva. Influence analysis in skew-BirnbaumSaunders regression models and applications. J. Appl. Stat., 38:1633-1649. Cited in page $2,36,43,45,53,63,64,73,74,77$

Spiegelhalter et al.(2002) D. J. Spiegelhalter, N. G. Best, B.P. Carlin and A. van der Linde. Bayesian measures of complexity and fit. Journal of The Royal Statistical Society Series $B, 64: 1-34$. Cited in page 44,65

Thomas and Cook(1990) W. Thomas and R. D. Cook. Assessing influence on predictions from generalized linear models. Technometrics, 32:59-65. Cited in page 7

Verbeke and Molenberghs(2000) G. Verbeke and G. Molenberghs. Linear mixed models for longitudinal data. Springer, New York. Cited in page 71

Vilca and Leiva(2006) F. Vilca and V. Leiva. A new fatigue life model based on family of skewelliptical distributions. Communications in Statistics-Theory and Methods, 35:229-244. Cited in page $2,3,17$

Vilca et al.(2010) F Vilca, A. Sanhueza, V. Leiva and G. Christakos. An extended BirnbaumSaunders model and its application in the study of environmental quality in Santiago, Chile. Stoch Environ Res Risk Assess, 24:771-782. Cited in page 12

Vilca et al.(2011) F. Vilca, L. Santana, V. Leiva and N. Balakrishnan. Estimation of extreme percentile in Birnbaum-Saunders distributions. Comp. Stat. Data Anal., 55:1665-1678. Cited in page 2, 11, 12, 13, 14, 15, 16, 19, 23, 25, 33, 36, 85 
Vilca et al.(2015) F. Vilca, C. B. Zeller and G. M. Cordeiro. The sinh-normal/independent nonlinear regression model. J. Appl. Stat., 42:1659-1676. Cited in page 36, 37, 43, 59, 62, 64,85

Vilca et al.(2017) F. Vilca, C. L. N. Azevedo and N. Balakrishnan. Bayesian inference for sinhnormal/independent nonlinear regression models. J. Appl. Stat., 44:2052-2074. Cited in page $36,37,38,41,42,43,62,63,64$

Wang and Genton(2006) J. Wang and M. Genton. The multivariate skew-slash distribution. Journal of Statistical Planning and Inference, 136:209-220. Cited in page 6

Xie and Wei(2007) F. C. Xie and B. C. Wei. Diagnostics analysis for log-Birnbaum-Saunders regression models. Comp. Stat. Data Anal., 51:4692-4706. Cited in page 63

Yamaguchi(1990) K. Yamaguchi. Generalized EM algorithm for model with contaminated error term. Proceedings of The Seven Japan and Korea Joint Conference of Statistics, pages 107-114. Cited in page 6

Zeller et al.(2010) C. B. Zeller, F. Vilca-Labra, Lachos V. H. and N. Balakrishnan. Influence analyses of skew-normal/independent linear mixed models. Comp. Stat. Data Anal., 54:1266-1280. Cited in page 4

Zhao and Lee(1998) Y. Zhao and A. Lee. Influence diagnostics for simultaneous equations models. Australian and New Zealand Journal Statistics, 40:345-357. Cited in page 7

Zhu and Lee(2001) H. Zhu and S. Lee. Local influence for incomplete-data models. J. R. Stat. Soc. $B, 63: 111-126$. Cited in page $4,7,8,9,63,67,70,71,83,85$

Zhu et al.(2001) H. Zhu, S. Lee, B. Wei and J. Zhou. Case-deletion measures for models with incomplete data. Biometrika, 88:727-737. Cited in page 4, 63, 67, 68, 83 